\title{
Biomimetic glucan particles with aggregation-induced emission characteristics for non-invasive monitoring transplant immune response
}

Tang Gao ${ }^{a, b, c \#}, Y a W^{a, b, c \#, ~ W e n y u a n ~ W a n g ~}{ }^{a, b, c}$, Cheng Deng ${ }^{a, b, c}$, Yihan Chen ${ }^{a, b, c}$, Luyang Yi ${ }^{a, b, c}$, Yishu Song ${ }^{a, b, c}$, Wenqu Li ${ }^{a, b, c}$, Lingling Xu ${ }^{a, b, c}$, Yuji Xie ${ }^{a, b, c}$, Lingyun $F^{a n g}{ }^{a, b, c}$, Qiaofeng Jin ${ }^{a, b, c^{*}}$, Li Zhang ${ }^{a, b, c^{*}}$, Ben Zhong Tang ${ }^{d^{*}}$, Mingxing Xie ${ }^{a, b, c^{*}}$

a Department of Ultrasound Medicine, Union Hospital, Tongji Medical College, Huazhong University of Science and Technology, Wuhan 430022, China.

b Hubei Province Clinical Research Center for Medical Imaging, Wuhan 430022, China.

${ }^{c}$ Hubei Province Key Laboratory of Molecular Imaging, Wuhan 430022, China.

d Department of Chemistry, Hong Kong Branch of Chinese National Engineering Research Center for Tissue Restoration and Reconstruction and Institute for Advanced Study, The Hong Kong University of Science and Technology (HKUST), Clear Water Bay, Kowloon, Hong Kong, China.

\# These authors contributed equally to this work

Corresponding authors E-mail: jin_qf@hust.edu.cn; zli429@hust.edu.cn; tangbenz@ust.hkxiemx@hust.edu.cn 


\section{Experimental section}

\section{Materials and instruments}

The

5-bromo-2-hydroxybenzaldehyde,

1,2-bis(4-methoxyphenyl)ethane-1,2-dione, (4-formylphenyl)boronic acid, aniline, ammonium acetate, trifluoroacetate(TFA), hydrogen peroxide solution $\left(\mathrm{H}_{2} \mathrm{O}_{2}\right)$, 2-aminophenol, 4-methylpyridine, iodoethane and hexamethylenetetramine(HMT) were purchased from Aladdin(China). $\mathrm{Pd}\left(\mathrm{PPh}_{3}\right)_{4}$, laminarin (LA), Calcofluor white stain, micro particles based on polystyrene $(500 \mathrm{~nm})$ and MCP-1 were purchased from Sigma-Aldrich.

\section{4,4,5,5-tetramethyl-2-(4-(1,2,2-triphenylvinyl)phenyl)-1,3,2-dioxaborolane(TPEB)}

and (4-(bis(4-methoxyphenyl)amino)phenyl)boronic acid(TPAB) were purchased from Alfa (Zhengzhou, China). Hydrochloric acid, sodium hydroxide, isopropano and acetone were purechased from Sinopharm Chemical Reagent (China). Baker's yeast was purchased from Angel (China). FK506 was purchased from MedchemExpress. Phosphate buffered saline (PBS), Dulbecco's Modified Eagle's Medium (DMEM) medium, fetal bovine serum (FBS), penicillin and streptomycin were purchased from Gibco. Cell counting kit-8 (CCK-8) and DAPI were purchased from Thermo Fisher Scientific. Nitric oxide and ROS Assay Kit were purchase from Beyotime. Alanine transaminase(ALT) assay kit , aspartate transaminase(AST) assay kit, blood urea nitrogen (BUN) assay kit and creatinie (CR)serum detection kit were purchased from Changchun Huili Biotech Co., Ltd(China). NK-92 cells was obtained from National collection of Authenticated Cell Cultures (Shang Hai). Other chemicals were of 
analytical grade and were used without further purification. Ultrapure water was supplied by Mill-Q Plus System (Millipore Corporation, USA).

${ }^{1} \mathrm{H}$ NMR and ${ }^{13} \mathrm{C}$ NMR spectra were measured by the Bruker ARX 400 NMR spectrometer or Bruker ARX 600 NMR spectrometer. SEM images of yeasts and glucan particles(GPs) were taken on scanning electron microscopy (Sirion 200, Netherlands). TEM images of yeasts and glucan particles(GPs)were performed on transmission electron microscope (Tecnai G2 20, Netherlands). High-resolution mass spectra (HRMS) were recorded on Orbitrap LC/MS (Thermo Fisher, Germany). XRD was recorded on Empyrean X-ray diffractometer (PANalytical B.V., Netherlands). Fourier transform infrared (FT-IR) spectra were obtained on VERTEX 70(Bruker). UV-Vis absorption spectra were measured on Lambda 35 (PerkinElmer). Solid fluorescent spectra were measured by FP-6500 (Jasco, Japan). Solution fluorescence emission spectrum were recored on LS55(PerkinElmer). Size and Zeta potential of samples were determined by Zestier Nano ZS ((Malvern). Fluorescence images of living cells were taken on a confocal laser scanning microscopy (ZEISS-LSM780, Germany). Living mice imaging was acquired with In-Vivo FX PRO imaging system (Bruker, Germany).

\section{Syntheses}

\subsection{Synthesis of 2-(benzo[d]thiazol-2-yl)-4-bromophenol (HBTBr)}

5-Bromo-2-hydroxybenzaldehyde (301.5 $\mathrm{mg}, 1.5 \mathrm{mmol})$ and 2-aminophenol (187.8 $\mathrm{mg}, 1.5 \mathrm{mmol}$ ) were dissolved in $5 \mathrm{~mL}$ anhydrous ethanol. Then $1 \mathrm{~mL}$ hydrochloric acid (37\%) and $40 \mu \mathrm{L} \mathrm{H}_{2} \mathrm{O}_{2}$ were added. The mixture was stirred under 
$37{ }^{\circ} \mathrm{C}$. After completion, the mixture was filterd and recrystalled to obtain pure product $\mathrm{HBTBr}$ with yiled 83.7\%. ${ }^{1} \mathrm{H} \mathrm{NMR}\left(400 \mathrm{MHz}, \mathrm{CDCl}_{3}\right), \delta(\mathrm{ppm}): 12.55(\mathrm{~s}, 1 \mathrm{H})$, 7.99 - $8.01(\mathrm{~d}, 1 \mathrm{H}), 7.92-7.94(\mathrm{~d}, 1 \mathrm{H}), 7.79-7.80(\mathrm{~d}, 1 \mathrm{H}), 7.51-7.55(\mathrm{~m}, 1 \mathrm{H}), 7.43-7.47$

$(\mathrm{m}, 2 \mathrm{H}) ;{ }^{13} \mathrm{C} \mathrm{NMR}\left(100 \mathrm{MHz}, \mathrm{CDCl}_{3}\right), \delta(\mathrm{ppm}): 167.77,157.01,151.63,135.31$, $132.61,130.48,126.93,125.95,122.37,121.63,119.81,111.02$.

\subsection{Synthesis of 3-(benzo[d]thiazol-2-yl)-5-bromo-2-hydroxybenzaldehyde} (HBTBrQ)

Compound HBTBr (100 mg, $0.33 \mathrm{mmol})$ was disolved in $8 \mathrm{~mL}$ TFA and then hexamethylenetetramine $(300 \mathrm{mg}, 2.14 \mathrm{mmol})$ was added. The reaction mixture was stirred for 12 hours under $120^{\circ} \mathrm{C}$ and the reaction was monitored by thin layer chromatography (TLC). After completion of the reaction, the reaction system was poured into $200 \mathrm{~mL}$ of aqueous solution, the $\mathrm{pH}$ of the system was adjusted to about 6 with $\mathrm{NaOH}$ solution, and the precipitate was filtered. After drying, it was separated and purified by silica gel column chromatography to obtain the compound HBTBrQ with a yield of $63.7 \%{ }^{1} \mathrm{H} \mathrm{NMR}\left(400 \mathrm{MHz}, \mathrm{CDCl}_{3}\right), \delta(\mathrm{ppm}): 10.48(\mathrm{~s}, 1 \mathrm{H}), 8.13(\mathrm{~s}, 1 \mathrm{H})$, 7.95-8.06 (m, 4H), 7.55-7.59 (t, $1 \mathrm{H}), 7.55-7.59(\mathrm{t}, 1 \mathrm{H}) ;{ }^{13} \mathrm{C} \mathrm{NMR}\left(100 \mathrm{MHz}, \mathrm{CDCl}_{3}\right)$, $\delta(\mathrm{ppm}): 159.38,151.25,136.39,127.20,126.38,122.62,121.73,111.64$.

\section{(4-(4,5-bis(4-methoxyphenyl)-1-phenyl-1H-imidazol-2-yl)phenyl)boronic acid}

Synthesis was carried out according to our previous literature report. ${ }^{1,2}$ Aniline $(0.9 \mathrm{~mL}, 10.0 \mathrm{mmol})$ and (4-formylphenyl)boronic acid $(1.5 \mathrm{~g}, 10.0 \mathrm{mmol})$ were dissolved in acetic acid $(20 \mathrm{~mL})$ and stirred for $1 \mathrm{hr}$ at room temperature. Benzil (2.10 
$\mathrm{g}, 10.00 \mathrm{mmol})$ and ammonium acetate $(3.6 \mathrm{~g}, 46.0 \mathrm{mmol})$ were added, subsequently. The mixture was heated at $120{ }^{\circ} \mathrm{C}$ overnight. After completion, the solution was poured into water. After neutralization, the mixture was filtered and washed with water. The residue was purified by silica gel column chromatography using petroleum ether and ethyl acetate $(1: 1 \mathrm{v} / \mathrm{v})$ as the elution solvent to give compound (4-(4,5-bis(4-methoxyphenyl)-1-phenyl-1H-imidazol-2-yl)phenyl)boronic acid as white powder $(1.5 \mathrm{~g}$, yield $=32 \%) .{ }^{1} \mathrm{H}$ NMR $\left(400 \mathrm{MHz}, \mathrm{DMSO}-\mathrm{d}_{6}\right), \delta(\mathrm{ppm}): 8.33(\mathrm{~s}$, 2H), 7.64-7.66(s, 2H), 7.42-7.44 (s, 2H), 7.29-7.34 (m, 5H), 7.23-7.24 (m, 2H), 7.14-7.1 ${ }^{5}(\mathrm{~d}, 2 \mathrm{H}), 6.83-6.86(\mathrm{~m}, 4 \mathrm{H}), 3.72(\mathrm{~s}, 6 \mathrm{H}) ;{ }^{13} \mathrm{C} \mathrm{NMR}\left(100 \mathrm{MHz}, \mathrm{CDCl}_{3}\right)$, $\delta(\mathrm{ppm}): 159.41,158.37,146.08,137.39,137.29,134.20,132.93,132.26,130.68$ $130.10,129.59,129.20,129.06,127.95,127.57,127.46,122.91,114.35,114.09$, 55.46 .

5-(benzo[d]thiazol-2-yl)-4'-(4,5-bis(4-methoxyphenyl)-1-phenyl-1H-imidazol-2-yl)4-hydroxy-[1,1'-biphenyl]-3-carbaldehyde (HBTTPIQ)

In $\quad 50 \quad \mathrm{~mL} \quad$ round-bottom (4-(4,5-bis(4-methoxyphenyl)-1-phenyl-1H-imidazol-2-yl)phenyl)boronic acid (90.4 mg, $0.19 \mathrm{mmol})$, Compound HBTBrQ $(63.2 \mathrm{mg}, 0.19 \mathrm{mmol})$ and $\mathrm{Pb}\left(\mathrm{PPh}_{3}\right)_{4}(20 \mathrm{mg}$, $0.017 \mathrm{mmol})$ were dissolved in $\operatorname{DMF}(4 \mathrm{~mL})$ and $\mathrm{K}_{2} \mathrm{CO}_{3}$ solution $(1.5 \mathrm{mmol}, 1 \mathrm{~mL})$ under the protection of $\mathrm{N}_{2}$, then the mixture was heated to $80{ }^{\circ} \mathrm{C}$ and stirred for $12 \mathrm{~h}$. After that, the reaction mixture was cooled to room temperature and exacted with DCM (40 mL x 3), then the organic layer was dried over $\mathrm{NaSO}_{4}$ and concentrated. 
The crude product was purified by silica gel chromatography with petroleum ether /ethyl acetate $(3: 1, \mathrm{v} / \mathrm{v})$ to obtain compound HBTTPIQ as orange power (44.3 $\mathrm{mg}$, 34\%). ${ }^{1} \mathrm{H}$ NMR (600 MHz, $\left.\mathrm{CDCl}_{3}\right), \delta(\mathrm{ppm}): 10.57,(\mathrm{~s}, 1 \mathrm{H}), 8.23(\mathrm{~s}, 1 \mathrm{H}), 8.10(\mathrm{~s}, 1 \mathrm{H})$, 8.04-8.05 (d, 1H), 7.94-7.96 (d, 1H), 7.51-7.57 (m, 8H), 7.45-7.48 (m, 1H), 7.30-7.35(m, 3H), 7.09-7.11(m, 2H), 7.05-7.06(d, 2H), 6.82-6.84(d, 2H), 6.76-6.78(d 2H), 3.78-3.80(d, 6H); ${ }^{13} \mathrm{C}$ NMR (150 MHz, $\left.\mathrm{CDCl}_{3}\right), \delta(\mathrm{ppm}): 159.98,159.22,158.48$, $151.49,145.85,138.22,137.29,132.39,129.42,129.25,128.55,128.52,128.39$, $127.03,126.27,126.07,122.79,122.50,121.69,113.88,113.67,55.22,55.15$.

(E)-4-(2-(5-(benzo[d]thiazol-2-yl)-4'-(4,5-bis(4-methoxyphenyl)-1-phenyl-1H-imi dazol-2-yl)-4-hydroxy-[1,1'-biphenyl]-3-yl) vinyl)-1-ethylpyridin-1-ium (HBTTPIP)

1-ethyl-4-methylpyridin-1-ium (11.4 mg, $0.046 \mathrm{mmol})$, intermediate HBTTPIQ (21 mg, $0.031 \mathrm{mmol}$ ) and $10 \mu \mathrm{L}$ piperidine were added in $10 \mathrm{~mL}$ ethanol. The mixture was refluxed at $980{ }^{\circ} \mathrm{C}$ for $8 \mathrm{~h}$ and subsequently concentrated to dryness. The residues were separated by silica gel chromatography with $\mathrm{CH}_{2} \mathrm{Cl}_{2} / \mathrm{MeOH}(30: 1$, v/v) to give HBTPIP as orange red powder (14.8 mg, yield: 60.3\%). ${ }^{1} \mathrm{H}$ NMR (600 MHz, DMSO-d $\left.\mathrm{d}_{6}\right), \delta(\mathrm{ppm}): 13.43,(\mathrm{~s}, 1 \mathrm{H}), 8.99-9.0(\mathrm{~d}, 2 \mathrm{H}), 8.21-8.28(\mathrm{~m}, 6 \mathrm{H}), 8.15-8.17(\mathrm{~d}$, 1H), 7.83-7.86(d, 1H), 7.79-7.80(d, 2H), 7.63-7.64(m, 1H), 7.54-7.58(m, 3H), 7.47-7.48(d, 2H), 7.41-7.42(m, 3H), 7.37-7.38(m, 2H), 7.18-7.20(d, 2H), 6.87-6.91(m, $4 \mathrm{H}), \quad 4.54-4.58(\mathrm{q}, \quad 2 \mathrm{H}), \quad 3.74-3.75(\mathrm{~d}, \quad 6 \mathrm{H}), \quad 1.54-1.57(\mathrm{t}, \quad 3 \mathrm{H}) ;{ }^{13} \mathrm{C} \quad \mathrm{NMR} \quad(150$ MHz,DMSO-d $\left.{ }_{6}\right), \delta(\mathrm{ppm}): 168.38,159.63,158.33,158.04,153.39,151.19,144.65$, 
$135.26,133.32,133.01,131.02,130.33,130.12,129.82,129.30,128.57,128.24$ $127.72,126.66,125.82,124.44,122.93,122.59,118.63,114.45,114.28,55.81,55.53$, 55.51,16.65. HRMS (ESI, m/z): [M-I] ${ }^{+}$calcd for $\mathrm{C}_{51} \mathrm{H}_{41} \mathrm{~N}_{4} \mathrm{O}_{3} \mathrm{~S}^{+}$789.2894, found $[\mathrm{M}-\mathrm{I}+\mathrm{H}]^{2+} / 2 \mathrm{z} 395.1478$.

\subsection{Synthesis of 5-(benzo[d]thiazol-2-yl)-4'-(bis(4-methoxyphenyl)} amino)-4-hydroxy-[1,1'-biphenyl]-3-carbaldehyde (HBTTPAQ)

In a $50 \mathrm{~mL}$ round-bottom flask, (4-(bis(4-methoxyphenyl)amino)phenyl)boronic acid (69.8 mg, $0.2 \mathrm{mmol})$, Compound HBTBrQ (66.6 mg, $0.2 \mathrm{mmol}) \quad$ and $\mathrm{Pb}\left(\mathrm{PPh}_{3}\right)_{4}$ (20 mg, $0.017 \mathrm{mmol}$ ) were dissolved in $\operatorname{DMF}(4 \mathrm{~mL})$ and $\mathrm{K}_{2} \mathrm{CO}_{3}$ solution (1.5 mmol, $1 \mathrm{~mL}$ ) under the protection of $\mathrm{N}_{2}$, then the mixture was heated to $75^{\circ} \mathrm{C}$ and stirred for $12 \mathrm{~h}$. After that, the reaction mixture was cooled to room temperature and exacted with DCM $(40 \mathrm{~mL} \times 3)$, then the organic layer was dried over $\mathrm{NaSO}_{4}$ and concentrated. The crude product was purified by silica gel chromatography with petroleum ether /ethyl acetate (10:1, v/v) to obtain compound HBTTPAQ as red power (34.6 mg, yield : 31\%). ${ }^{1} \mathrm{H}$ NMR $\left(400 \mathrm{MHz}, \mathrm{CDCl}_{3}\right), \delta(\mathrm{ppm}): 10.58(\mathrm{~s}, 1 \mathrm{H})$, $8.26(\mathrm{~s}, 1 \mathrm{H}), 8.06-8.10(\mathrm{~m}, 2 \mathrm{H}), 7.95-7.97(\mathrm{~d}, 1 \mathrm{H}), 7.54-7.58(\mathrm{~m}, 1 \mathrm{H}), 7.44-7.49(\mathrm{~m}$, 3H), 7.01-7.12 (m, 7H), 6.85-6.88 (d, 4H), 3.82(s, 6H). ${ }^{13} \mathrm{C}$ NMR (100 MHz, $\left.\mathrm{CDCl}_{3}\right)$, $\delta(\mathrm{ppm}): 159.31,156.05,151.40,149.19,147.07,145.45,140.69,132.31,129.92$ $127.20,126.99,126.73,125.99,124.37,123.48,122.43,121.65,120.66,119.10$, $119.00,118.88,114.78,114.07,55.52$.

2.7 Synthesis of (E)-4-(2-(5-(benzo[d]thiazol-2-yl)-4'-(bis(4-methoxyphenyl) amino)-4-hydroxy-[1,1'-biphenyl]-3-yl) vinyl)-1-ethylpyridin-1-ium (HBTTPAP) 
1-ethyl-4-methylpyridin-1-ium (36 mg, $0.14 \mathrm{mmol}$ ), intermediate HBTTPAQ (31.3 $\mathrm{mg}, 0.056 \mathrm{mmol}$ ) and $10 \mu \mathrm{L}$ piperidine were added in $10 \mathrm{~mL}$ ethanol. The mixture was refluxed at $95{ }^{\circ} \mathrm{C}$ for $10 \mathrm{~h}$ and subsequently concentrated to dryness. The residues were separated by silica gel chromatography with $\mathrm{CH}_{2} \mathrm{Cl}_{2} / \mathrm{MeOH}(20: 1$, v/v) to give HBTTPAP as orange red powder (25.4mg, yield: $57.4 \%) .{ }^{1} \mathrm{H}$ NMR (600 MHz, DMSO-d $\left.{ }_{6}\right), \delta(\mathrm{ppm}): 13.30(\mathrm{~s}, 1 \mathrm{H}), 8.98-8.99(\mathrm{~d}, 2 \mathrm{H}), 8.10-8.28(\mathrm{~m}, 7 \mathrm{H}), 7.82-7.85(\mathrm{~d}$, 1H), 7.56-7.65(m, 4H), 6.92-7.08(m, 10H), 4.55-4.56(q, 2H), 3.77(s, 6H), 1.55(t, 3H). HRMS (ESI, m/z): [M-I] ${ }^{+}$calcd for $\mathrm{C}_{42} \mathrm{H}_{36} \mathrm{~N}_{3} \mathrm{O}_{3} \mathrm{~S}^{+}$662.2472, found 662.24579.

\section{5-(benzo[d]thiazol-2-yl)-4-hydroxy-4'-(1,2,2-triphenylvinyl)-[1,1'-biphenyl]-3-car} baldehyde (HBTTPEQ)

In $\quad 50 \quad \mathrm{~mL} \quad$ round-bottom flask, 4,4,5,5-tetramethyl-2-(4-(1,2,2-triphenylvinyl)phenyl)-1,3,2-dioxaborolane (149.8 mg, $0.33 \mathrm{mmol})$, Compound HBTBrQ (108.8 mg, $0.33 \mathrm{mmol})$ and $\mathrm{Pb}\left(\mathrm{PPh}_{3}\right)_{4}(30 \mathrm{mg}$, $0.026 \mathrm{mmol}$ ) were dissolved in $\operatorname{DMF}(5 \mathrm{~mL})$ and $\mathrm{K}_{2} \mathrm{CO}_{3}$ solution $(2 \mathrm{mmol}, 1 \mathrm{~mL})$ under the protection of $\mathrm{N}_{2}$, then the mixture was heated to $75^{\circ} \mathrm{C}$ and stirred for $16 \mathrm{~h}$. After that, the reaction mixture was cooled to room temperature and exacted with DCM $(50 \mathrm{~mL} \times 3)$, then the organic layer was dried over $\mathrm{NaSO}_{4}$ and concentrated. The crude product was purified by silica gel chromatography with petroleum ether /ethyl acetate (15:1, v/v) to obtain compound HBTTPEQ as orange power $(81.2 \mathrm{mg}$, yield: $42 \%) .{ }^{1} \mathrm{H}$ NMR (600 MHz, DMSO-d $\left.{ }_{6}\right), \delta(\mathrm{ppm}): 10.56(\mathrm{~s}, 1 \mathrm{H}), 8.25(\mathrm{~s}, 1 \mathrm{H})$, 8.05-8.09(m, 1H), 7.94-7.95(d, 1H), 7.54-7.57(m, 1H), 7.45-7.48(m, 1H), 7.39-7.40(d, 
2H), 7.08-7.13(m, 15H), 6.88-6.90(m, 2H), 6.56-6.58(m, 2H). ${ }^{13} \mathrm{C}$ NMR (150 MHz, $\left.\mathrm{CDCl}_{3}\right), 159.78,154.10,151.37,144.01,143.92,143.90,143.66,143.63,143.35$, $141.47,140.46,140.25,140.18,136.49,136.33,132.74,132.54,132.08,131.41$, $131.38,131.35,131.34,127.89,127.79,127.72,127.69,127.62,126.60,126.39$, $126.27,125.86,122.46,121.69 .114 .61$.

(E)-4-(2-(5-(benzo[d]thiazol-2-yl)-4-hydroxy-4'-(1,2,2-triphenylvinyl)-[1,1'-biphe nyl]-3-yl) vinyl)-1-ethylpyridin-1-ium iodide (HBTTPEP)

1-ethyl-4-methylpyridin-1-ium (14.9 mg, $0.06 \mathrm{mmol})$, intermediate HBTTPEQ ( $25 \mathrm{mg}, 0.04 \mathrm{mmol}$ ) and $10 \mu \mathrm{L}$ piperidine were added in $10 \mathrm{~mL}$ ethanol. The mixture was refluxed at $95{ }^{\circ} \mathrm{C}$ for $10 \mathrm{~h}$ and subsequently concentrated to dryness. The residues were separated by silica gel chromatography with $\mathrm{CH}_{2} \mathrm{Cl}_{2} / \mathrm{MeOH}(20: 1$, v/v) to give HBTTPEP as orange powder (22 mg, yield: 67.3\%). ${ }^{1} \mathrm{H}$ NMR (600 MHz, DMSO-d 6 ), $\delta(\mathrm{ppm}): \quad 13.37(\mathrm{~s}, \quad 1 \mathrm{H}), \quad 8.99-9.00(\mathrm{~d}, 2 \mathrm{H}), \quad 8.22-8.27(\mathrm{~m}, 4 \mathrm{H}), \quad 8.12-8.19(\mathrm{~m}, 3 \mathrm{H})$, 7.82-7.85(d, 1H), 7.54-7.64(m, 4H), 7.00-7.21(m, 17H), 4.54-4.58(q, 2H), 1.54-1.57(t, 3H). ${ }^{13} \mathrm{C}$ NMR (150 MHz, DMSO-d 6 ), 168.36, 158.74, 158.52, 158.30, 158.08, $155.90,153.43,151.21,144.64,143.67,143.66,143.63,142.98,141.34,140.49$, $136.79,135.30,133.30,132.11,131.89,131.21,131.17,131.12,130.28,130.12$, $128.48,128.39,128.32,127.70,127.23,127.13,127.08,126.67,126.31,125.71$, 125.00, 124.43, 122.92, 122.57, 118.60, 55.80, 16.64. HRMS (ESI, m/z): [M-I] ${ }^{+}$ calcd for $\mathrm{C}_{48} \mathrm{H}_{37} \mathrm{~N}_{2} \mathrm{OS}^{+}$689.2621, found 689.2605.

\section{Particle size and zeta-potential}


Particle size and zeta-potential measurements were performed on a Malvern Zetasizer Nano ZS instrument with the measuring angle is $90^{\circ}$ at $25^{\circ} \mathrm{C}$.

\section{X-Ray Powder Diffraction}

The X-ray diffraction spectra of GPs and HBTTPEP/GPs were measured with a fast desktop powder diffractometer. The scanning region was set from $2 \theta=3^{\circ}$ to $2 \theta=50^{\circ}$ with a step interval of $0.02^{\circ}$ and a scan rate of $0.5^{\circ} \mathrm{min}^{-1}$.

\section{Fourier Transform Infrared Spectrum}

A small amount of HBTTPEP, GPs, HBTTPEP/GPs, as well as the mixture of HBTTPEP and GPs were mixed with $\mathrm{KBr}$ and pressed into pellets, then scanned from 4000 to $500 \mathrm{~cm}^{-1}$. The instrument worked with a spectral resolution ratio of $4 \mathrm{~cm}^{-1}$.

\section{Molecular dynamics simulation}

In order to obtain a reasonable structure and maintain a moderate amount of calculation, 3 repeating units were selected when constructing the model, each unit containing 6 main chain sugar rings and 1 side chain sugar ring. The single-stranded helical structure is constructed by using the monomer structural unit through the XLEAP module under Amber tools and the visualization software VMD. ${ }^{3}$ The constructed single-stranded helical structure is optimized by semi-empirical molecular orbital method (AM-1), and the optimized single-strand is adopted. The python script performs mathematical calculations and replicates the central axis of the single-stranded spiral to form a triple-helix structure. The newly constructed triple helix structure is optimized under vacuum through the molecular dynamics simulation by Gromacs 2019 packaged with Generalized Amber Force Field (GAFF). ${ }^{4}$ The 
optimized structure is used for subsequent simulation. Before docking, the structure optimization and conformation analysis of small molecules are performed, the structure and hydrogen atoms of the polysaccharide are optimized again, and the site analysis of the polysaccharide is performed to obtain the active site that may be docked. System 1 and System 2 were solvated in a $20 \times 20 \times 20 \mathrm{~nm}^{3}$ water box with a TIP3P water mode. ${ }^{5}$ MD simulations were performed with GROMACS 2019.2. Force field adopted general AMBER force field (GAFF); charge calculation used AM1-BCC partial charges. Chloride ion was used as a charge counter ion. The simulation steps were as follows: (1) Use steepest descent algorithm to minimize energy; (2) Simulated annealing temperature rise: $1 \mathrm{~ns}$, temperature from 0 to $310 \mathrm{~K}$ in 1 ns; (3) NVT system balance $10 \mathrm{~ns}$; (4) NPT system balance $10 \mathrm{~ns}$; (5) MD production simulation $200 \mathrm{~ns}$.

System composition of molecular dynamics simulation as follows:

\begin{tabular}{|c|c|c|c|c|c|c|c|}
\hline System & $\begin{array}{c}\text { Free } \\
\text { glucan }\end{array}$ & $\begin{array}{c}\text { Free } \\
\text { HBTTPEP }\end{array}$ & HBTTPEP : & $\begin{array}{c}\text { Com2 } \\
\text { HBTTPEP-glucan } \\
\text {-HBTTPEP }-1: 1: 1\end{array}$ & Water & $\mathrm{Cl}^{-}$ & Total atoms \\
\hline System 1 & 6 & 6 & & & 58198 & 6 & 183138 \\
\hline System 2 & 2 & 2 & 2 & 57620 & 8 & 181588 \\
\hline
\end{tabular}

\section{Cell culture}

RAW264.7 mouse macrophage cells was obtained from Zhong Qiao Xin Zhou Biotechnology Co., Ltd. (Shanghai, China). The cells were cultured in DMEM medium containing $10 \%$ fetal bovine serum (FBS), $100 \mathrm{UmL}^{-1}$ penicillin, and 100 $\mu g \mathrm{~mL}^{-1}$ streptomycin in a humidified atmosphere at $37{ }^{\circ} \mathrm{C}$ with $5 \% \mathrm{CO}_{2}$. Extracted $\mathrm{T}$ cells from spleen were cultured in RPMI-1640 medium containing 10\% fetal bovine 
serum (FBS), $100 \mathrm{UmL}^{-1}$ penicillin, and $100 \mu \mathrm{gmL}^{-1}$ streptomycin in a humidified atmosphere at $37{ }^{\circ} \mathrm{C}$ with $5 \% \mathrm{CO}_{2}$. NK-92 cells were cultred in NK cell media [MEM media supplemented with horse serum (12.5\%), FBS (12.5\%), L-glutamine (2 mM), folic acid $(20 \mu \mathrm{M})$, inositol $(200 \mu \mathrm{M})$ and 2-mercaptoethanol $(100 \mu \mathrm{M})]$ in a humidified atmosphere at $37^{\circ} \mathrm{C}$ with $5 \% \mathrm{CO}_{2}$.

\section{Classification for acute rejection of skin.}

The grafted skin was harvested from allograft and allograft, then washed with ice-cold PBS, fixed in 4\% paraformaldehyde overnight, and then embedded in paraffin. After slicing, hematoxylin, and eosin (H\&E) staining were performed. The grade of rejection of skin was evaluated according to the Banff classification. ${ }^{6}$ The Banff classification for cell-mediated acute rejection of skin was divided into 5 grades: grade 0 , no or rare inflammatory infiltrates; grade I, mild perivascular infiltration, with no involvement of the overlying epidermis; grade II, moderate to severe perivascular inflammation with or without mild epidermal or adnexal involvement (limited to spongiosis and exocytosis), and no epidermal dyskeratosis or apoptosis; grade III, dense inflammation and epidermal involvement with epithelial apoptosis, and dyskeratosis or keratinolysis; and grade IV, necrotizing acute rejection, with frank necrosis of epidermis or other skin structures.

\section{Real-time PCR}

The mRNA expression levels of CD68, MCP-1 and CSF-1 were quantified using real-time PCR. Briefly, RNA was extracted from the grafted skin and healthy skin. using TRIzol reagent (Invitrogen, Carlsbad, CA, USA). cDNA was synthesized from 
the RNA and amplified. Results were analysed using the $2^{-\Delta \Delta \mathrm{Ct}}$ method. The primer sequences for quantitative RT-PCR were designed as follows :

\begin{tabular}{cllc}
\hline Gene & Primer & \multicolumn{1}{c}{ Sequence (5'-3') } & PCR Products \\
\hline \multirow{2}{*}{$\beta$-actin } & Forward & CACGATGGAGGGGCCGGACTCATC & \multirow{2}{*}{ 240bp } \\
& Reverse & TAAAGACCTCTATGCCAACACAGT & \\
\multirow{2}{*}{ MCP-1 } & Forward & CAGCAGGTGTCCCAAAGAAG & 248bp \\
& Reverse & AAGTGCTTGAGGTGGTTGTG & \\
CD68 & Forward & CTAGTCCAAGGTCCAAGGGG & $205 \mathrm{bp}$ \\
& Reverse & GAGAGAGACAGGTGGGGATG & \\
& Forward & TGCTAAGTGCTCTAGCCGA & 191bp \\
\hline
\end{tabular}


1) 2-Hydroxyphenyl benzothiazole(HBT) derivatives
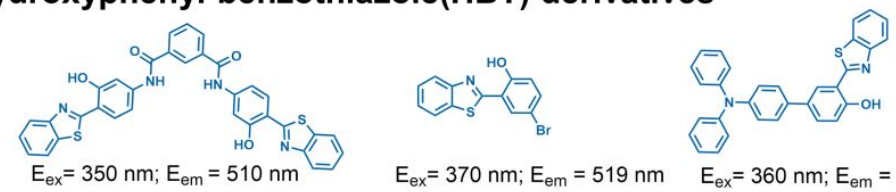

$E_{e x}=370 \mathrm{~nm} ; E_{e m}=519 \mathrm{~nm}$

$E_{e x}=360 \mathrm{~nm} ; E_{e m}=600 \mathrm{~nm}$
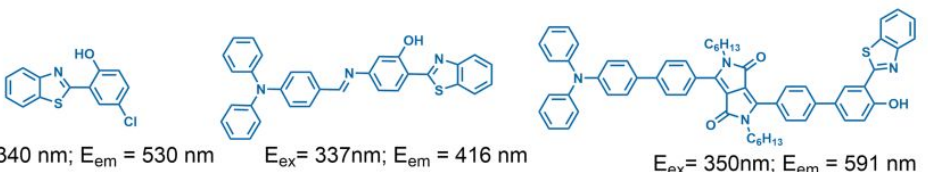

$E_{e x}=340 \mathrm{~nm} ; E_{e m}=530 \mathrm{~nm} \quad E_{e x}=337 n m ; E_{e m}=416 n m$

$E_{e x}=350 \mathrm{~nm} ; E_{e m}=591 \mathrm{~nm}$
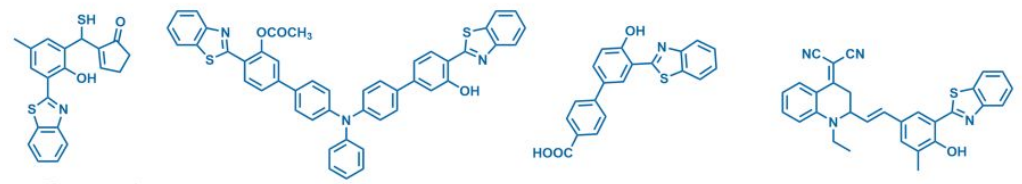

$E_{e x}=350 \mathrm{~nm} ; E_{e m}=540 \mathrm{~nm} \quad E_{e x}=380 \mathrm{~nm} ; E_{e m}=511 n m \quad E_{e x}=365 n m ; E_{e m}=551 n m \quad E_{e x}=460 n m ; E_{e m}=620 n m$

2) Salicylaldehyde-based Schiff-base molecules

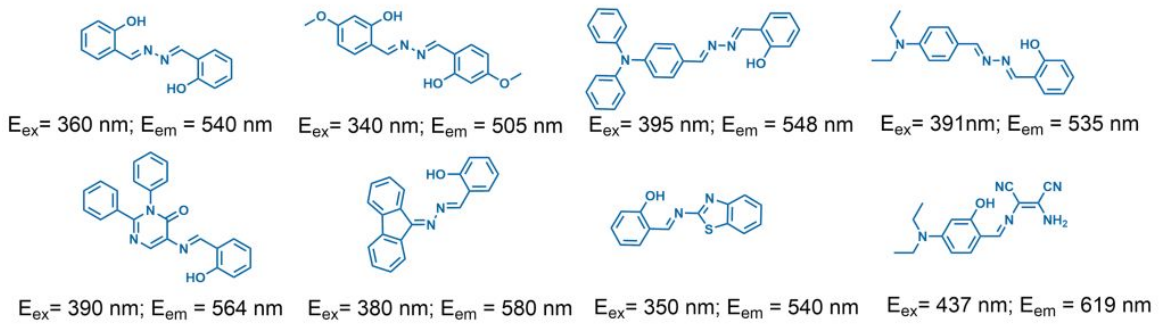

3) Chalcone derivative

4) 2-(2-Hydroxyphenyl) quinazolin-4(3H)-one(HPQ) derivatives

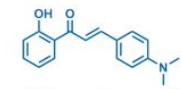

$E_{e x}=430 \mathrm{~nm} ; E_{e m}=640 \mathrm{~nm}$
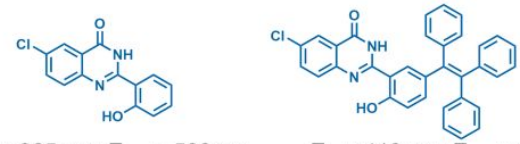

$E_{e x}=365 \mathrm{~nm} ; E_{e m}=500 \mathrm{~nm}$

$E_{e x}=410 \mathrm{~nm} ; E_{e m}=550 \mathrm{~nm}$

5)Tetraphenyl ethylene-based hybrid units
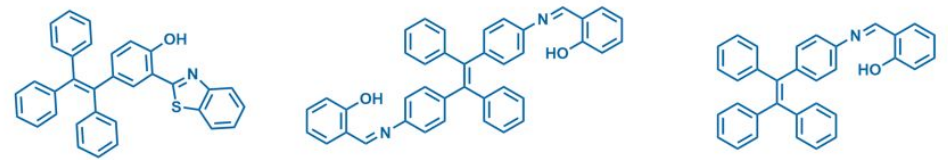

$$
E_{e x}=350 \mathrm{~nm} ; E_{e m}=558 \mathrm{~nm} \quad E_{e x}=370 \mathrm{~nm} ; E_{e m}=540 \mathrm{~nm} \quad E_{e x}=440 \mathrm{~nm} ; E_{e m}=579 n m
$$
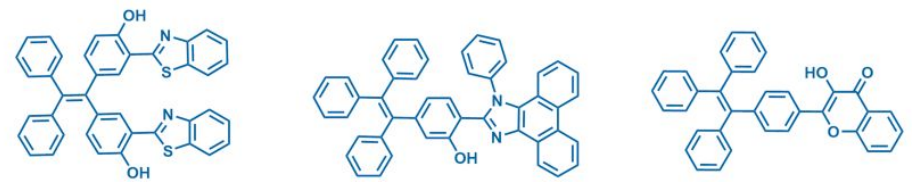

$$
E_{e x}=350 \mathrm{~nm} ; E_{e m}=555 \mathrm{~nm} \quad E_{e x}=370 \mathrm{~nm} ; E_{e m}=474 \mathrm{~nm} \quad E_{e x}=452 \mathrm{~nm} ; E_{e m}=550 \mathrm{~nm}
$$

Scheme S1. Previously reported fluorophores with character of AIE and ESIPT. 


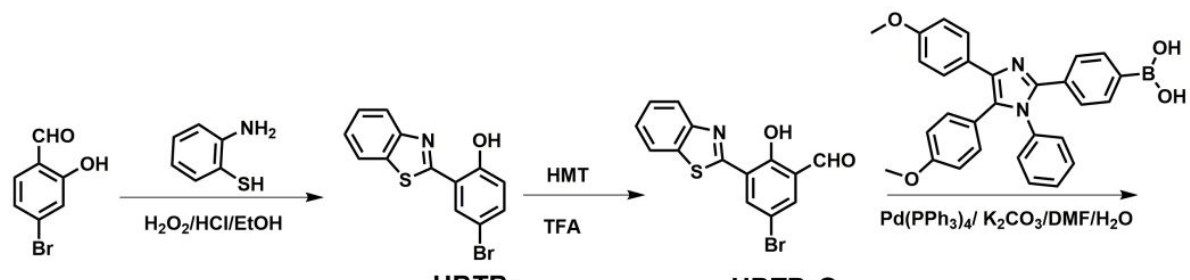
$\mathrm{HBTBr}$ HBTBrQ
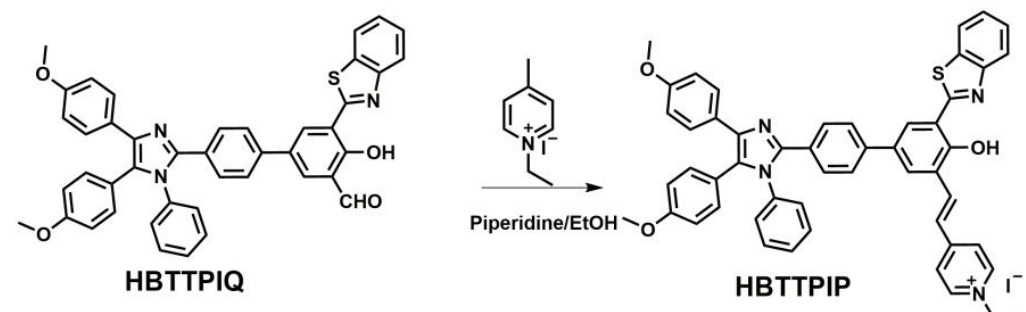

Scheme S2. Synthetic route of HBTTPIP.
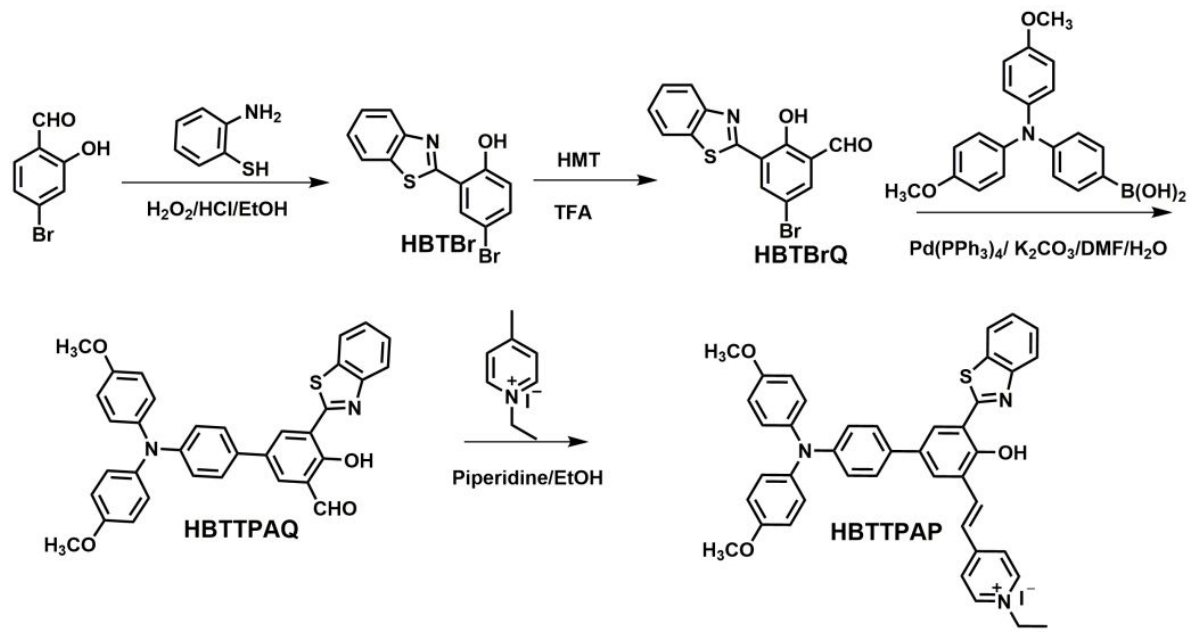

Scheme S3. Synthetic route of HBTTPAP.

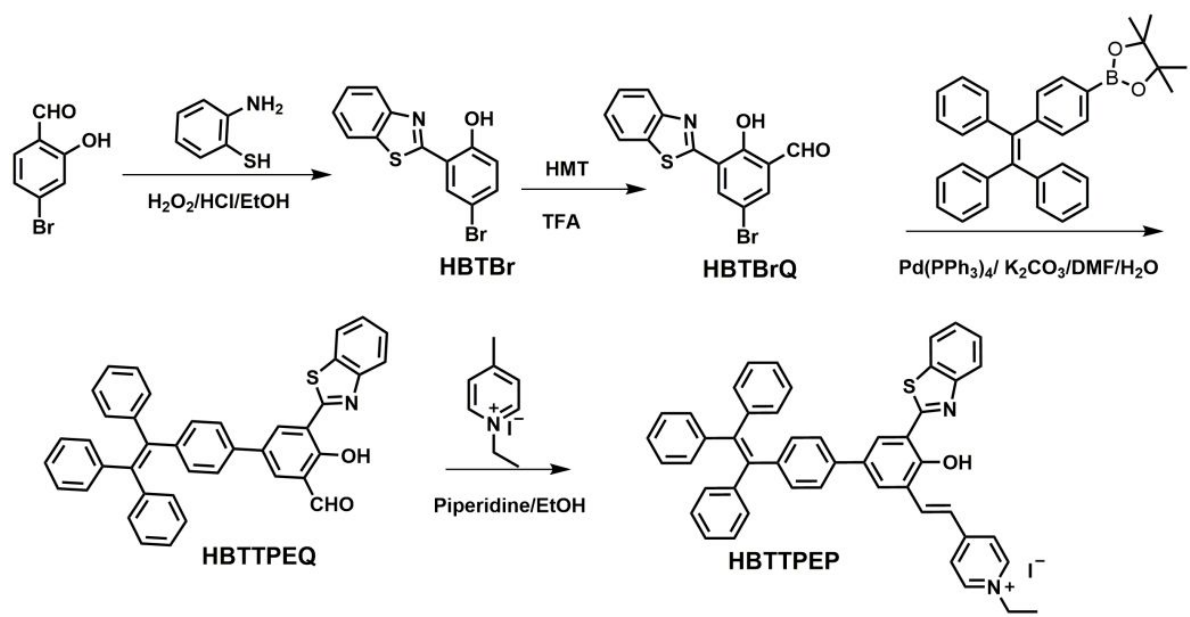

Scheme S4. Synthetic route of HBTTPEP. 

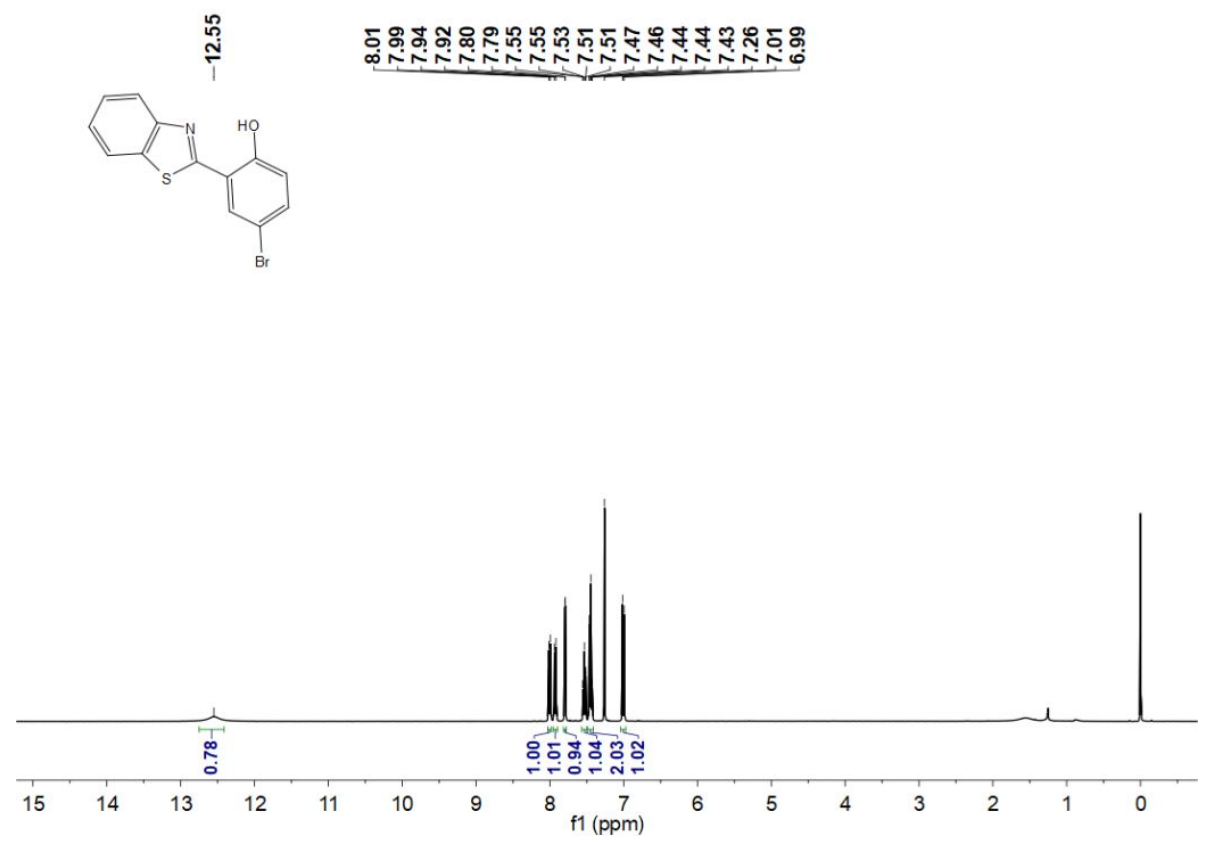

Figure S1. ${ }^{1} \mathrm{HNMR}$ spectrum of compound $\mathrm{HBTBr}$ in $\mathrm{CDCl}_{3}$.

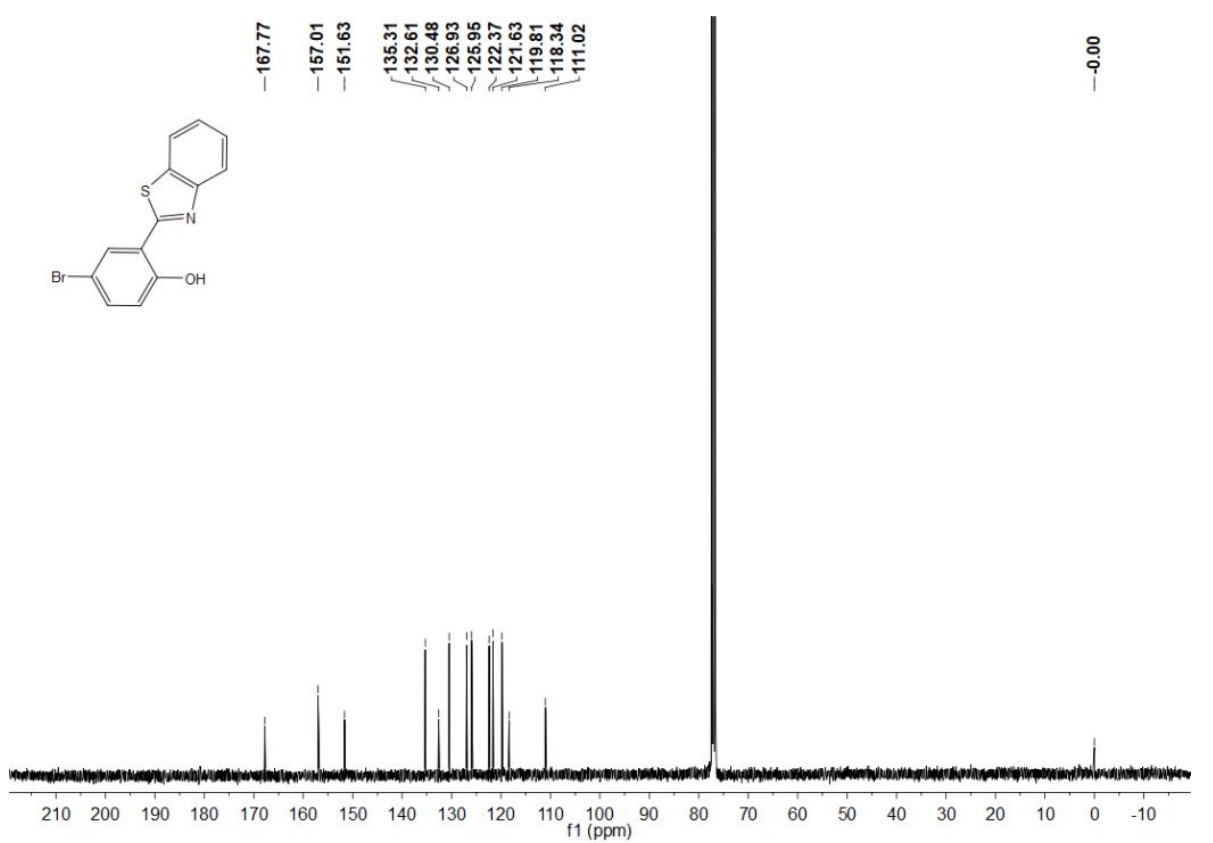

Figure S2. ${ }^{13} \mathrm{C}$ NMR spectrum of compound $\mathrm{HBTBr}$ in $\mathrm{CDCl}_{3}$. 


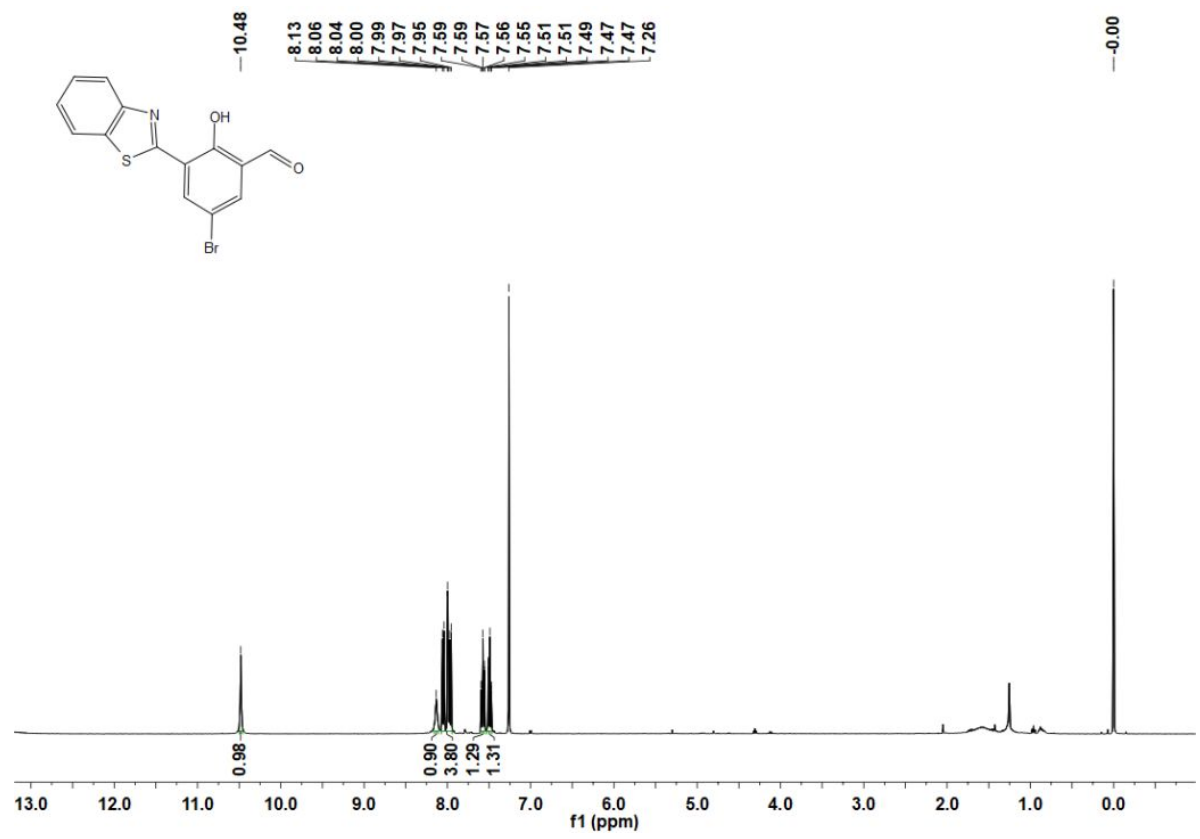

Figure S3. ${ }^{1} \mathrm{H}$ NMR spectrum of compound $\mathrm{HBTBrQ}$ in $\mathrm{CDCl}_{3}$

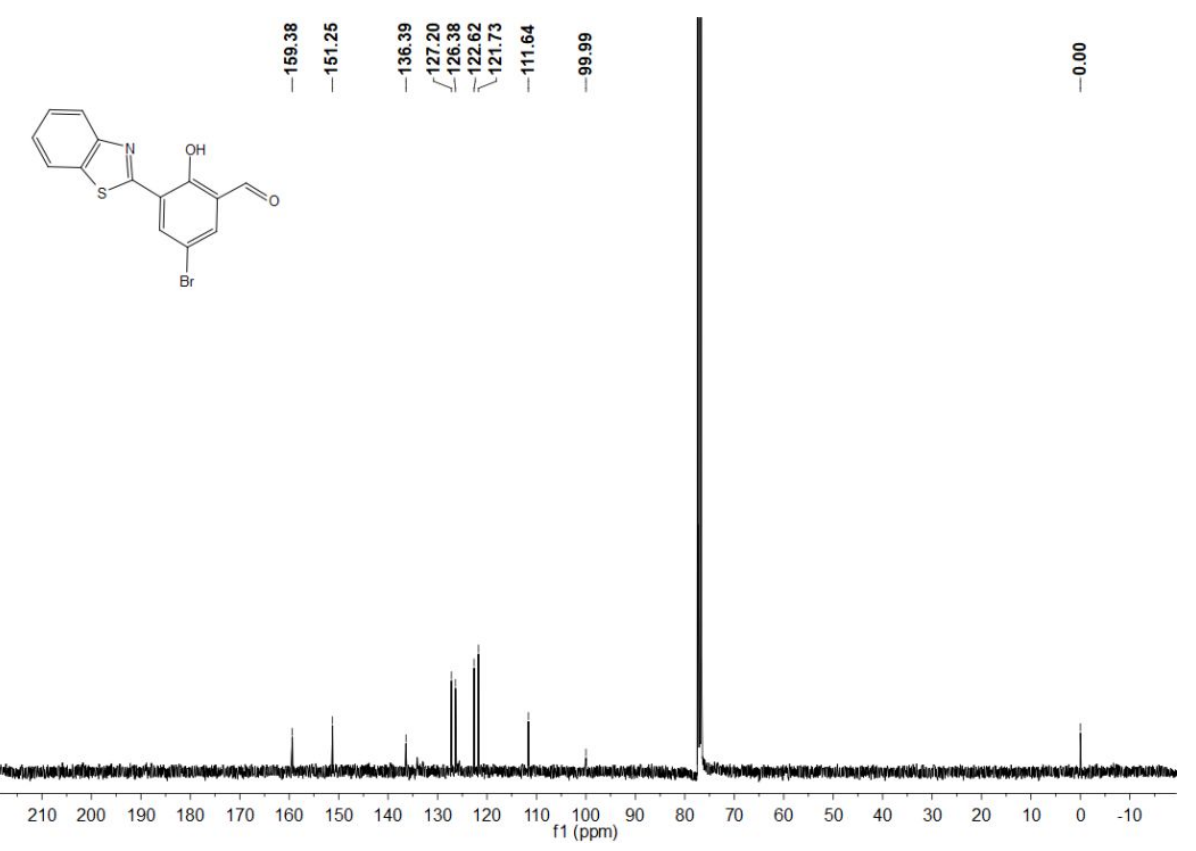

Figure S4. ${ }^{13} \mathrm{C}$ NMR spectrum of compound $\mathrm{HBTBrQ}$ in $\mathrm{CDCl}_{3}$. 


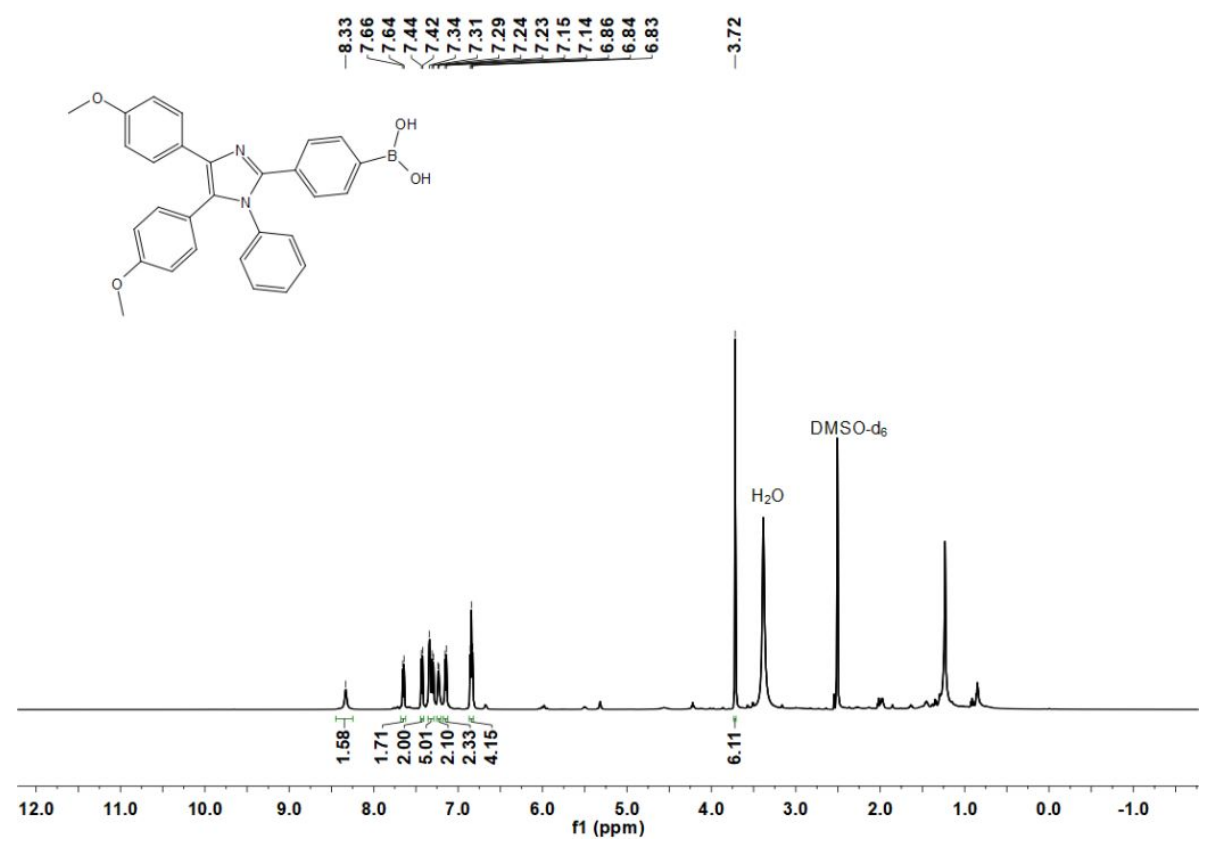

$\begin{array}{llllll}\text { Figure } & \text { S5. } & { }^{1} \mathrm{H} & \text { NMR } & \text { spectrum }\end{array}$ (4-(4,5-bis(4-methoxyphenyl)-1-phenyl-1H-imidazol-2-yl) phenyl) boronic acid in DMSO-d $\mathrm{d}_{6}$

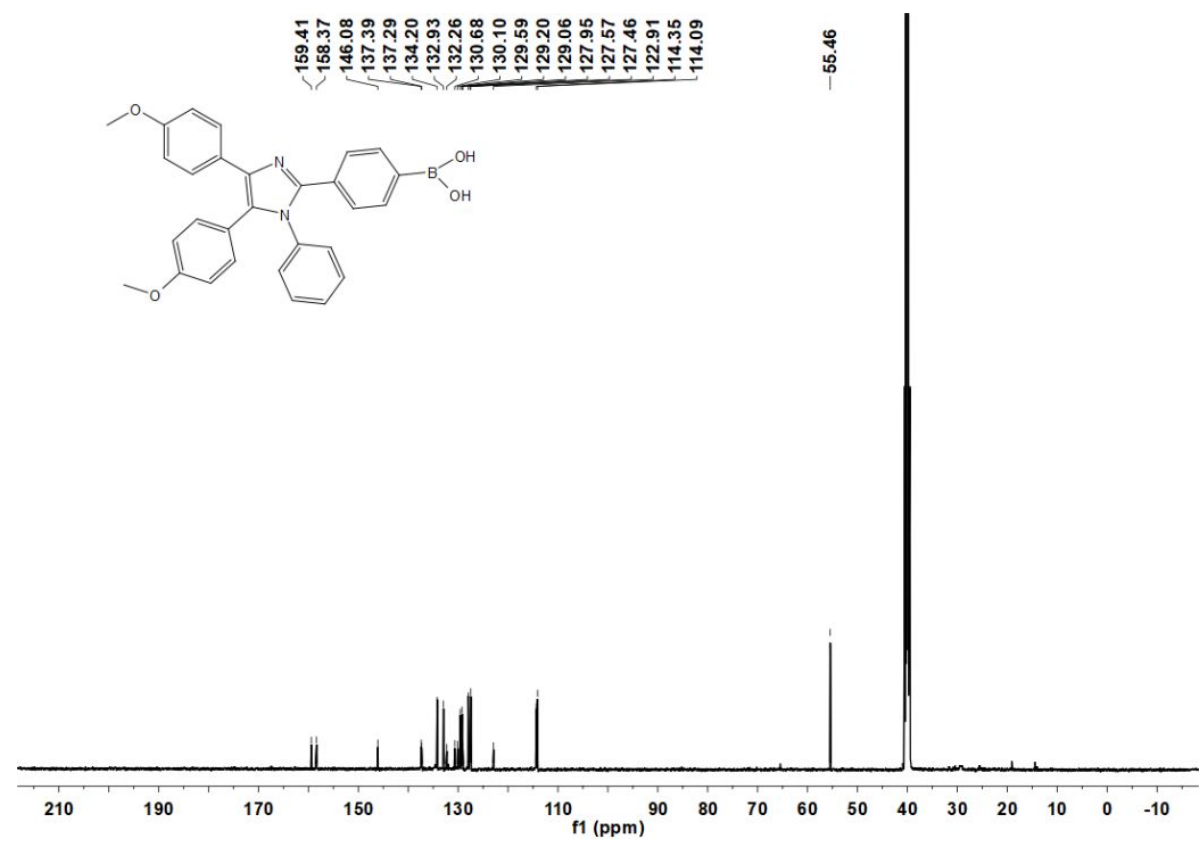

$\begin{array}{lllll}\text { Figure } & \text { S6. } & { }^{13} \mathrm{C} & \mathrm{NMR} & \text { spectrum }\end{array}$ (4-(4,5-bis(4-methoxyphenyl)-1-phenyl-1H-imidazol-2-yl) phenyl) boronic acid in DMSO-d 


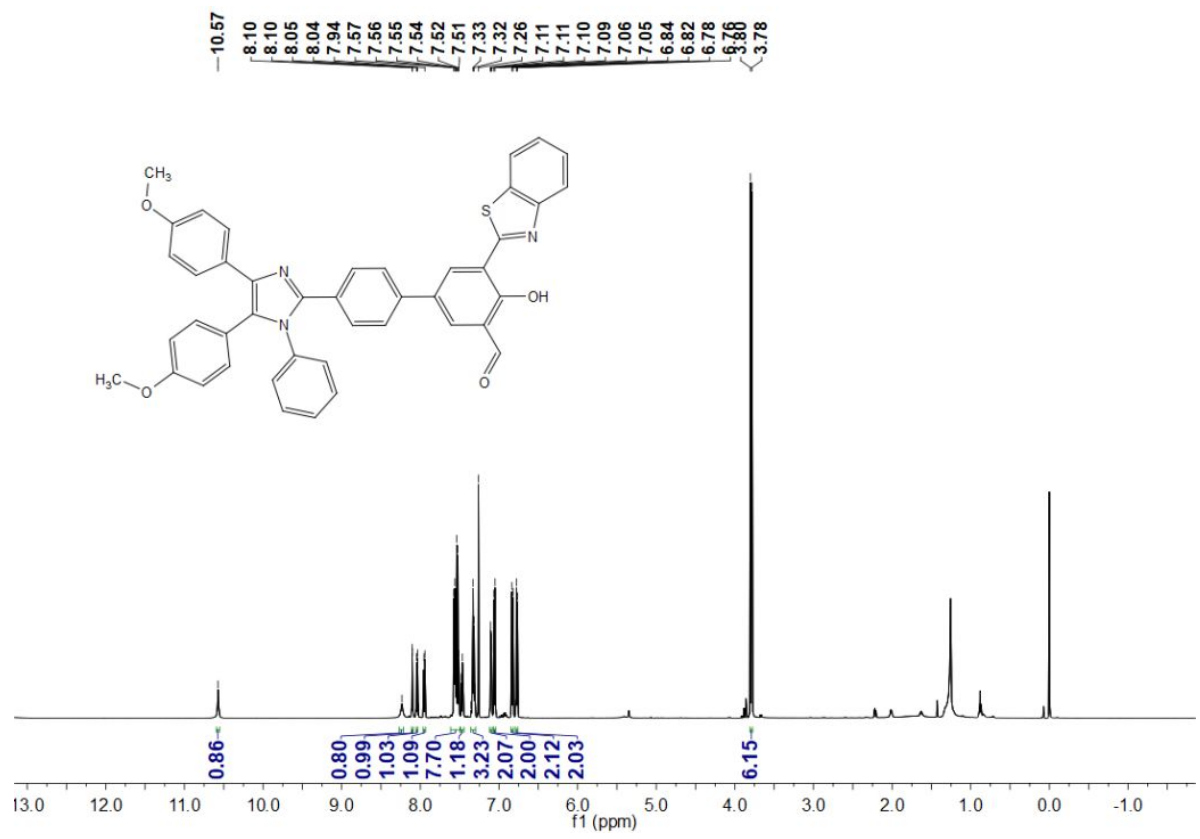

Figure S7. ${ }^{1} \mathrm{H}$ NMR spectrum of compound HBTTPIQ in $\mathrm{CDCl}_{3}$.

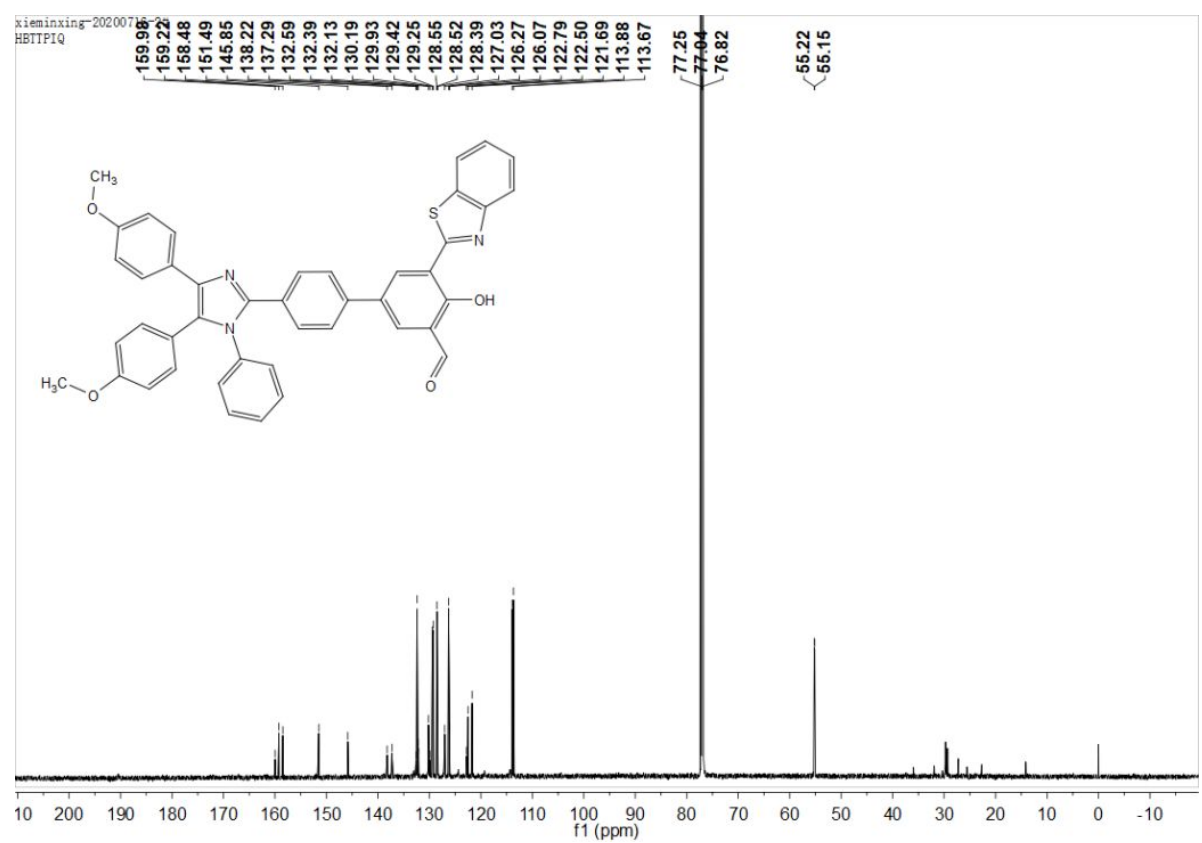

Figure S8. ${ }^{13} \mathrm{C}$ NMR spectrum of compound HBTTPIQ in $\mathrm{CDCl}_{3}$. 


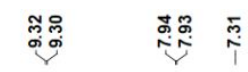

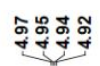

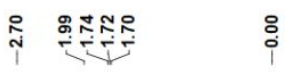

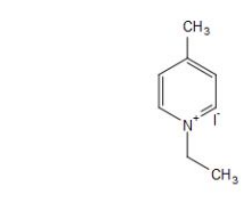

$\mathrm{CH}_{\mathrm{CH}_{3}}^{\mathrm{NH}_{3}}$

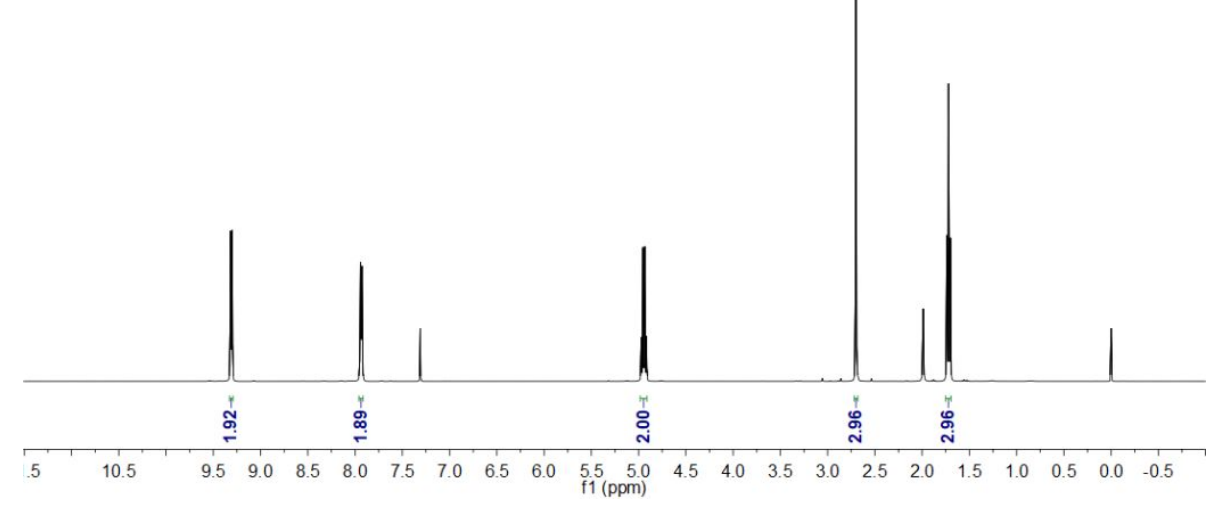

Figure S9. ${ }^{1} \mathrm{H}$ NMR spectrum of compound 1-ethyl-4-methylpyridin-1-ium iodide in $\mathrm{CDCl}_{3}$.

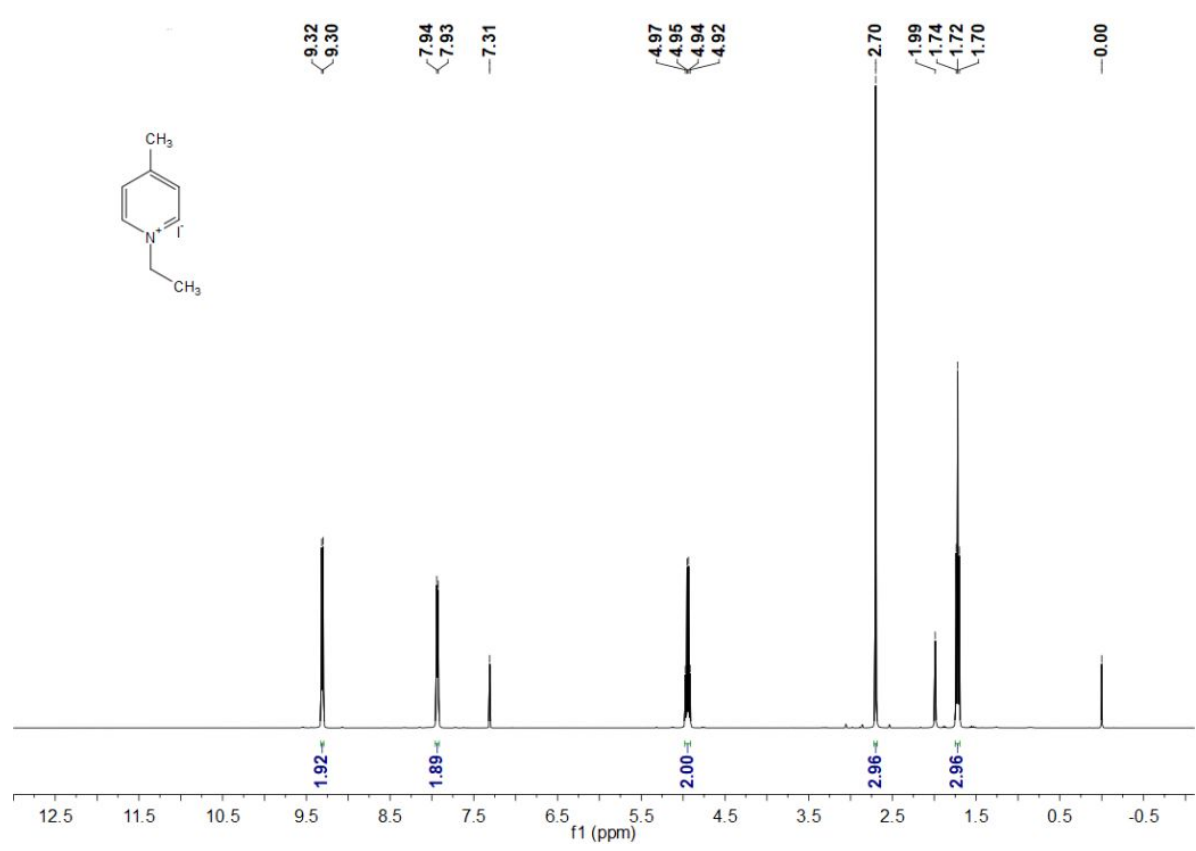

Figure S10. ${ }^{13} \mathrm{C}$ NMR spectrum of compound 1-ethyl-4-methylpyridin-1-ium iodide in $\mathrm{CDCl}_{3}$. 


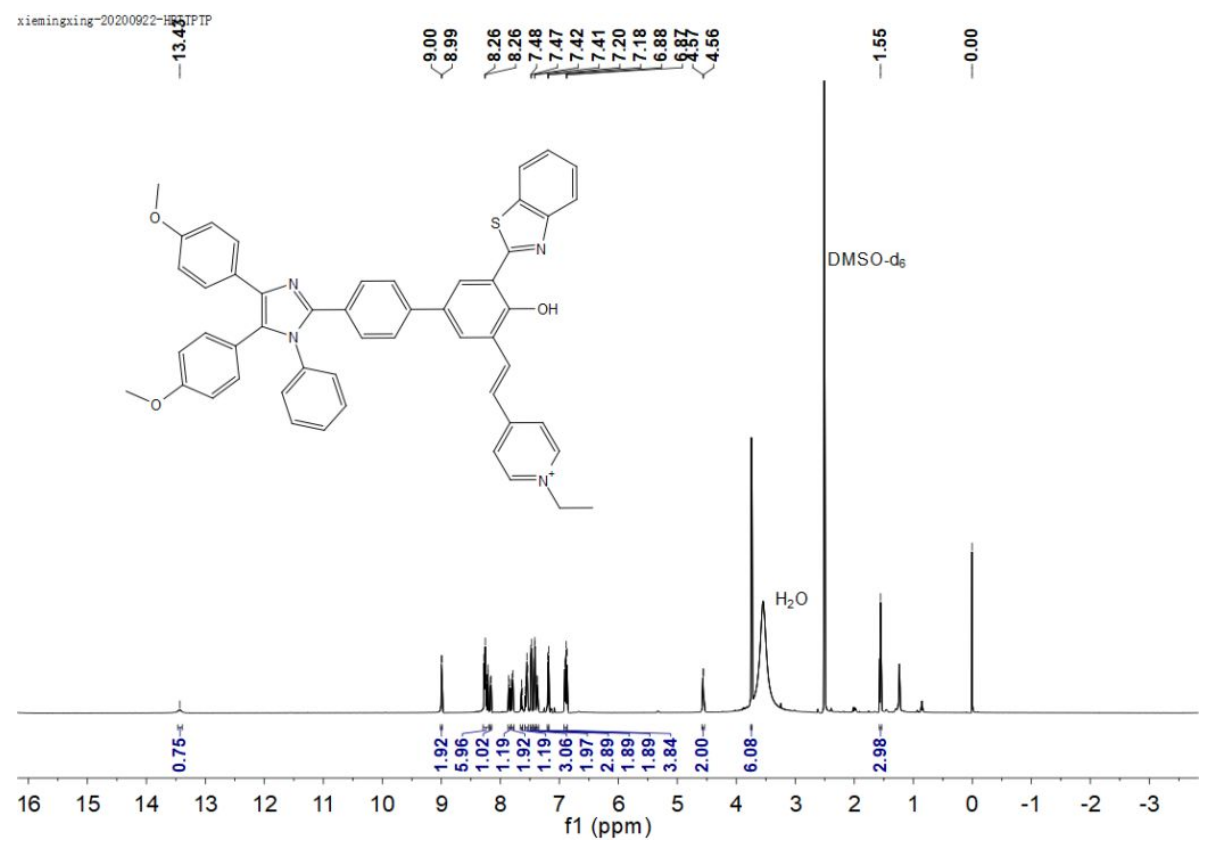

Figure S11. ${ }^{1} \mathrm{H}$ NMR spectrum of compound HBTTPIP in DMSO- $\mathrm{d}_{6}$.

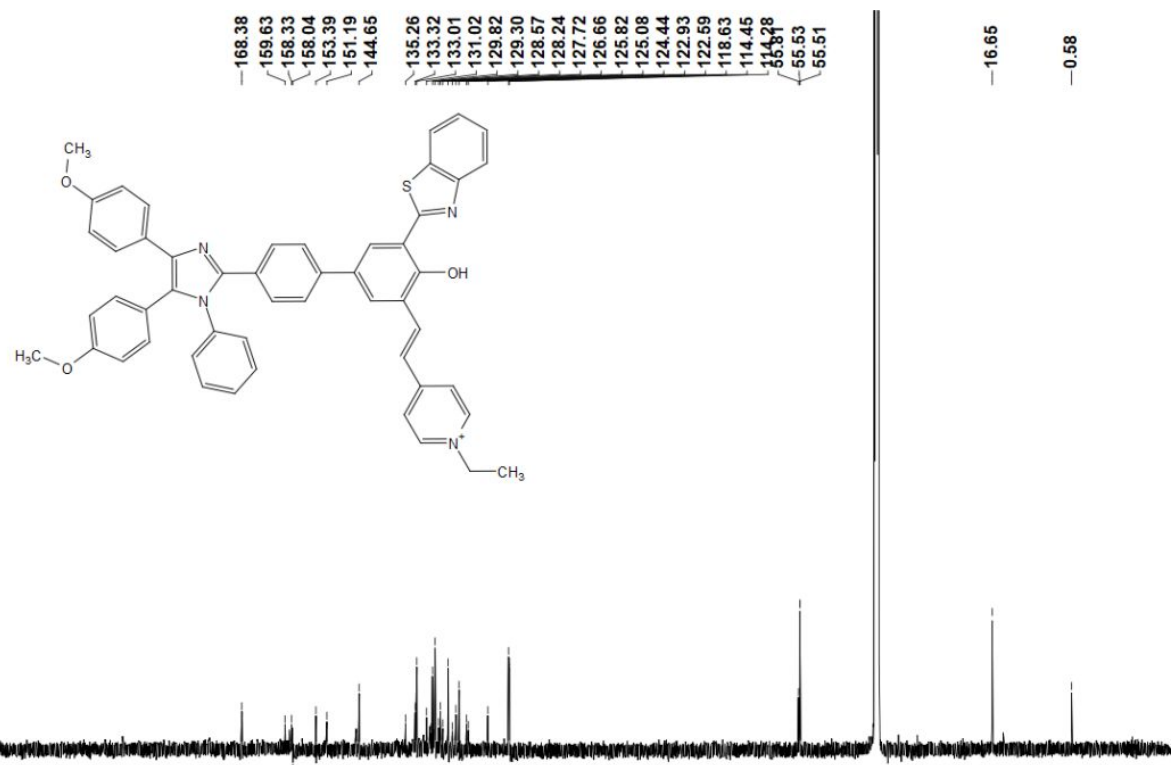

$\begin{array}{llllllllllllllllllllllllll}210 & 200 & 190 & 180 & 170 & 160 & 150 & 140 & 130 & 120 & 110 & 100 & 90 & 80 & 70 & 60 & 50 & 40 & 30 & 20 & 10 & 0 & -10\end{array}$

Figure S12. ${ }^{13} \mathrm{C}$ NMR spectrum of compound HBTTPIP in DMSO-d 6 . 


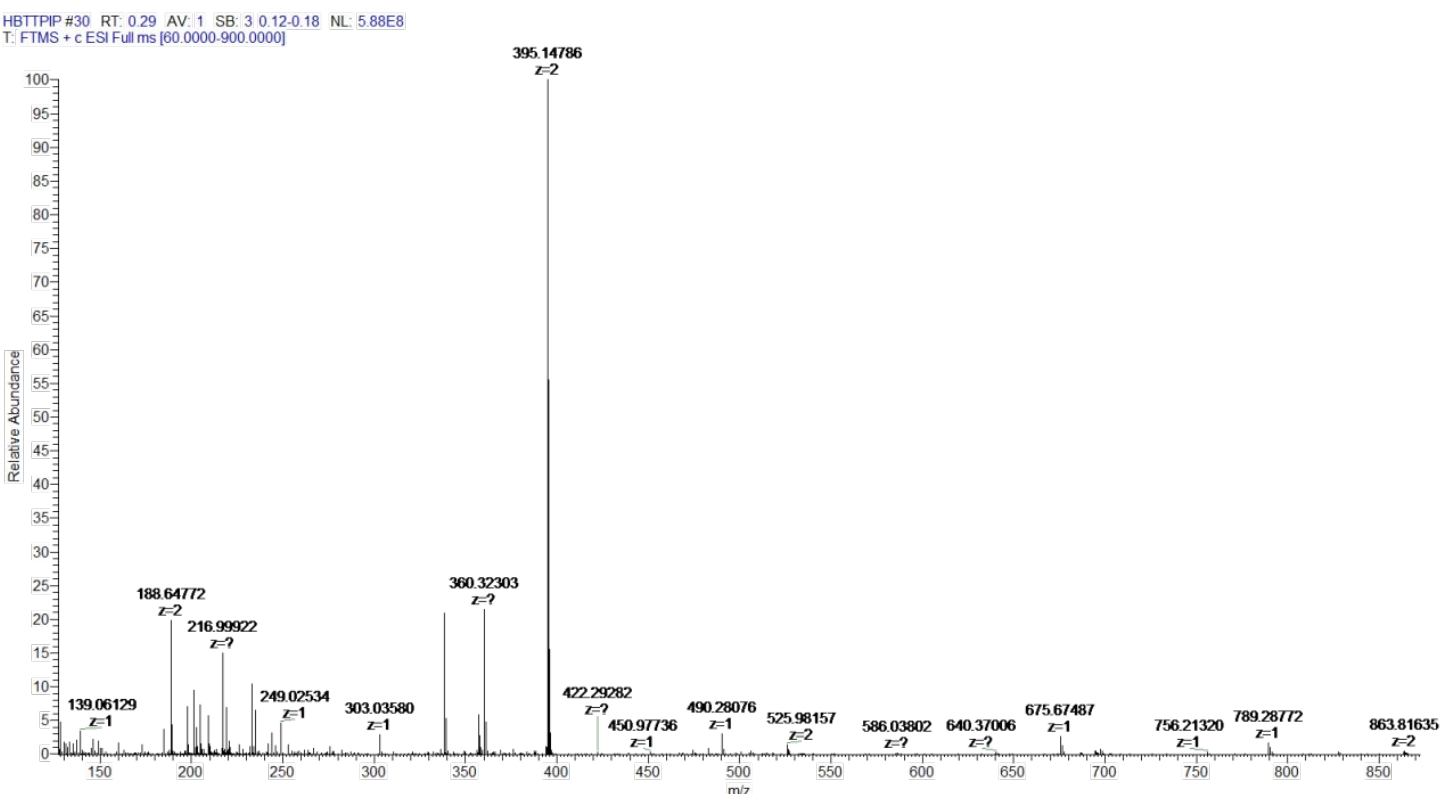

Figure S13. HRMS of compound HBTTPIP.

\section{:}

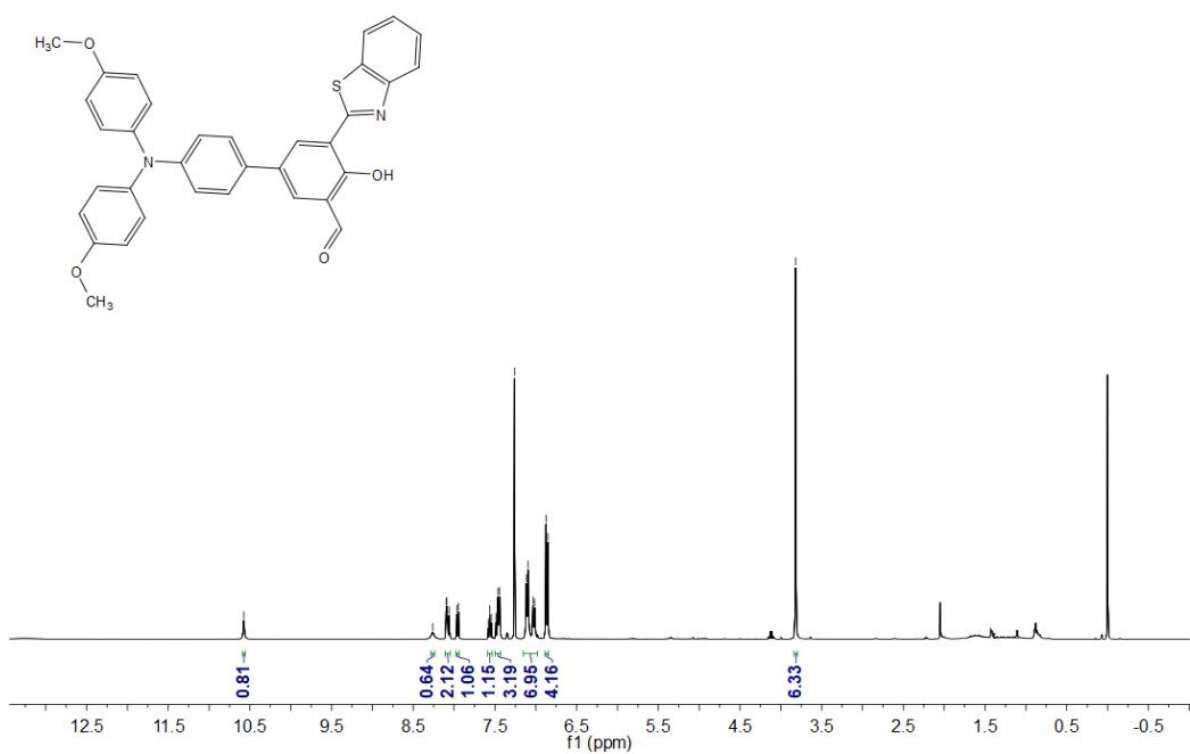

Figure S14. ${ }^{1} \mathrm{H}$ NMR spectrum of compound HBTTPAQ in $\mathrm{CDCl}_{3}$. 


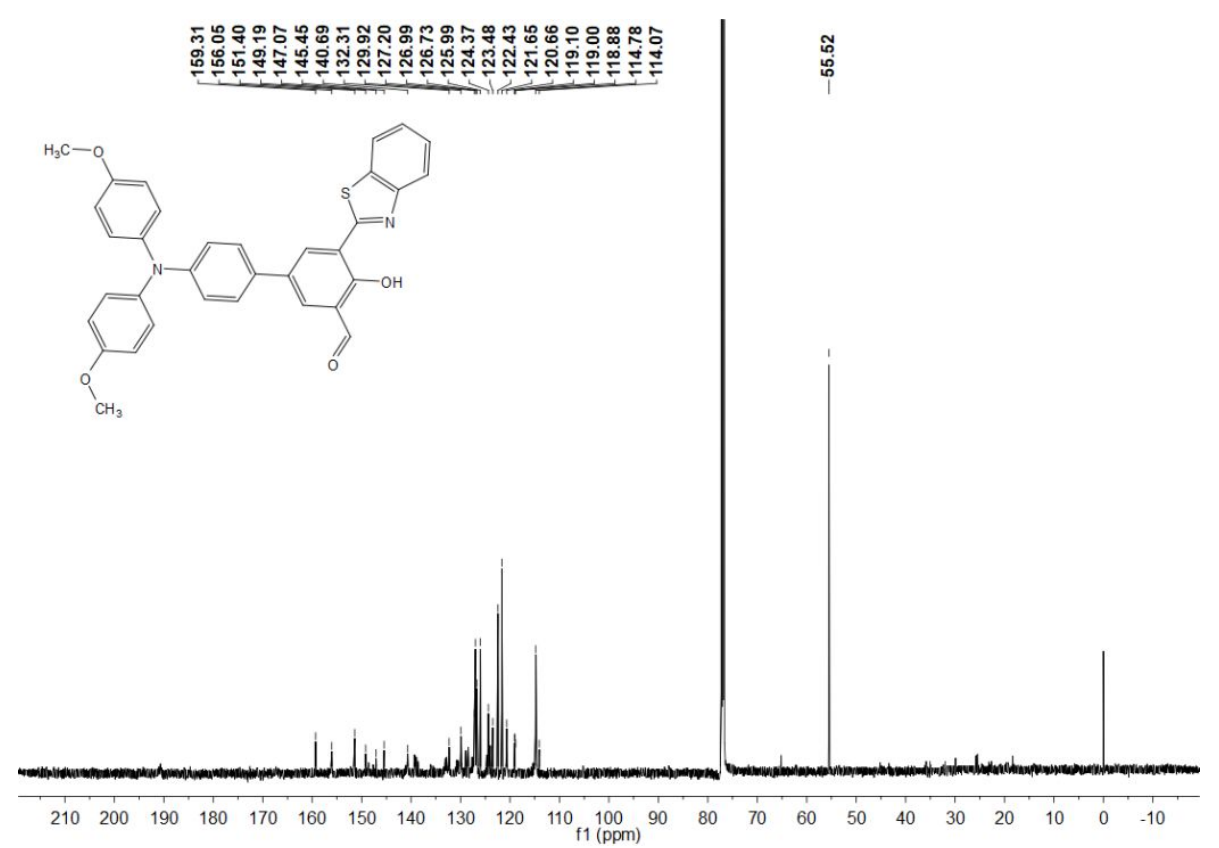

Figure S15. ${ }^{13} \mathrm{C}$ NMR spectrum of compound HBTTPAQ in $\mathrm{CDCl}_{3}$.

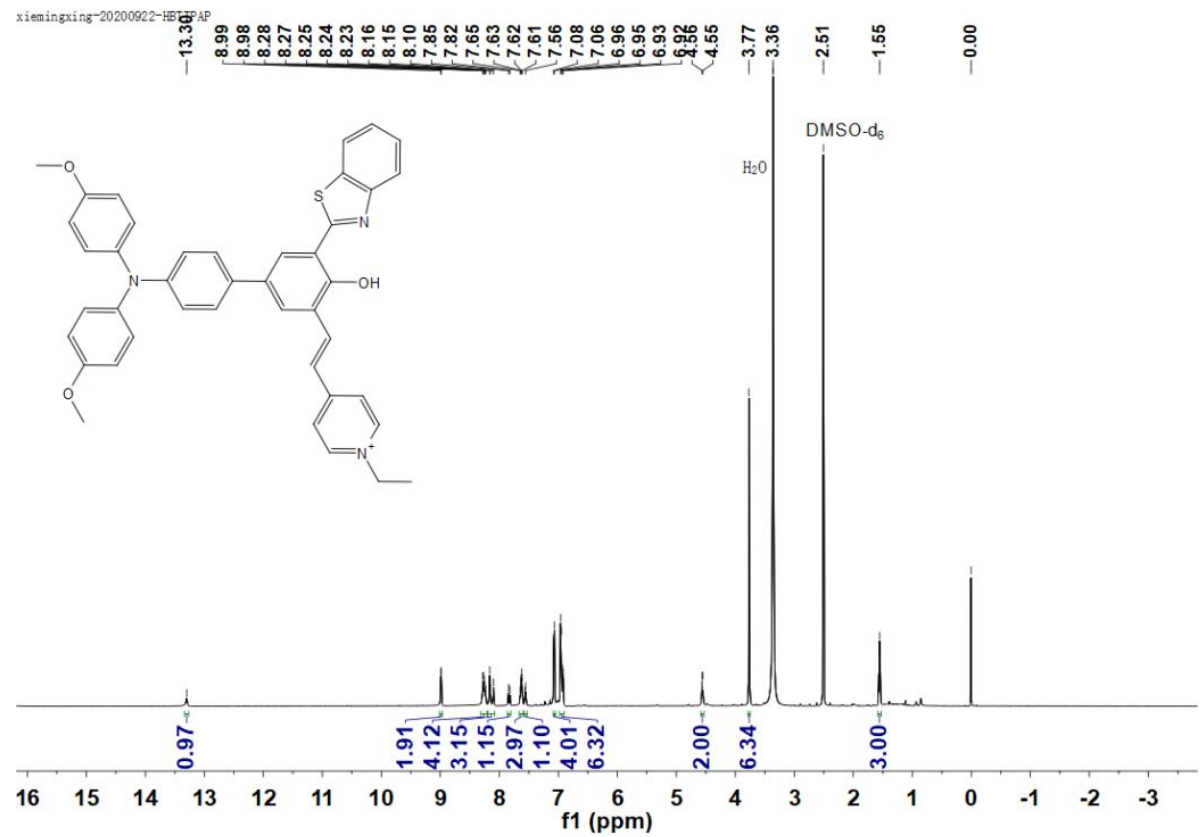

Figure S16. ${ }^{1} \mathrm{H}$ NMR spectrum of compound HBTTPAP in DMSO-d ${ }_{6}$. 


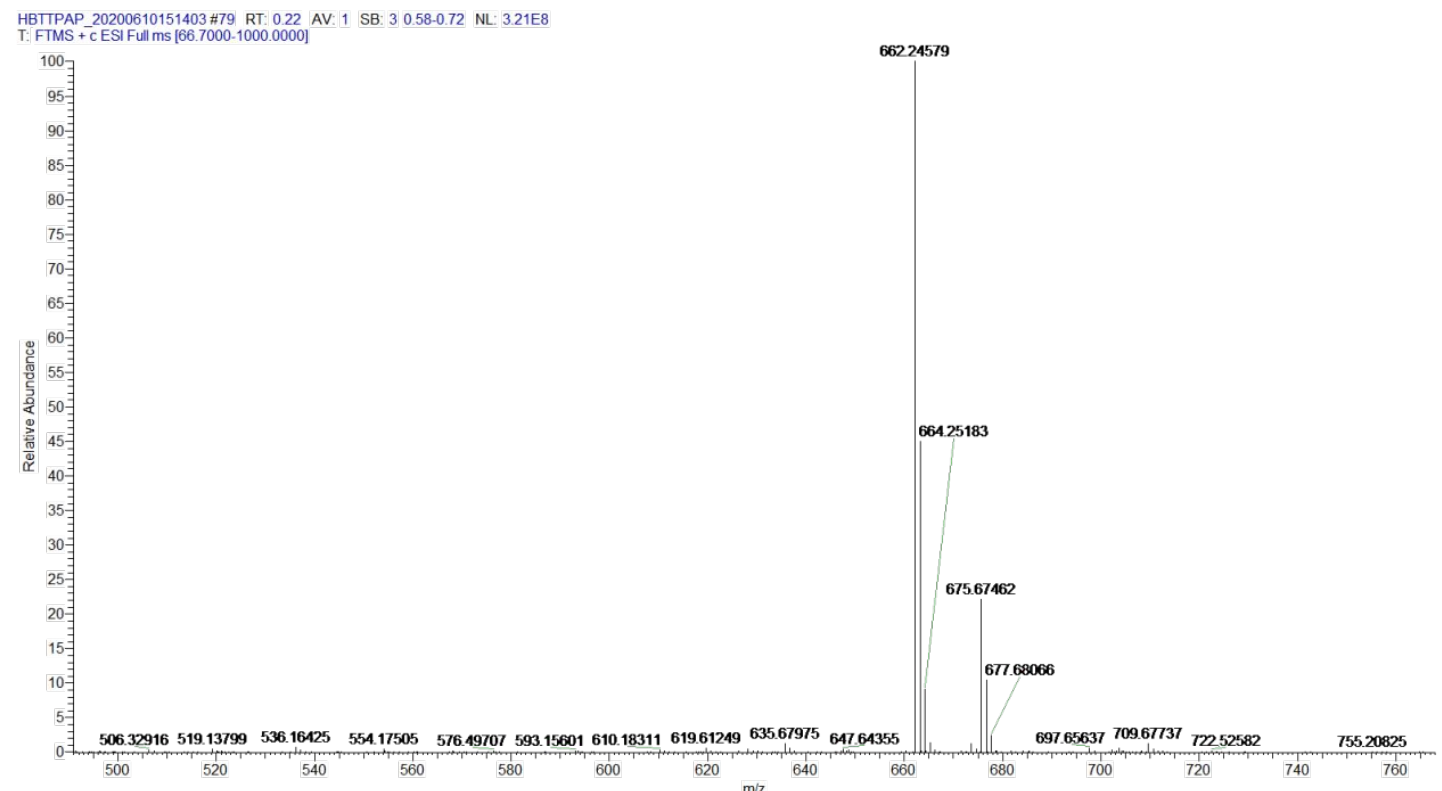

Figure S17. HRMS of compound HBTTPAP.

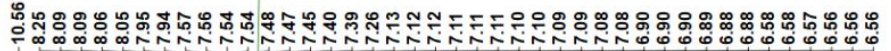

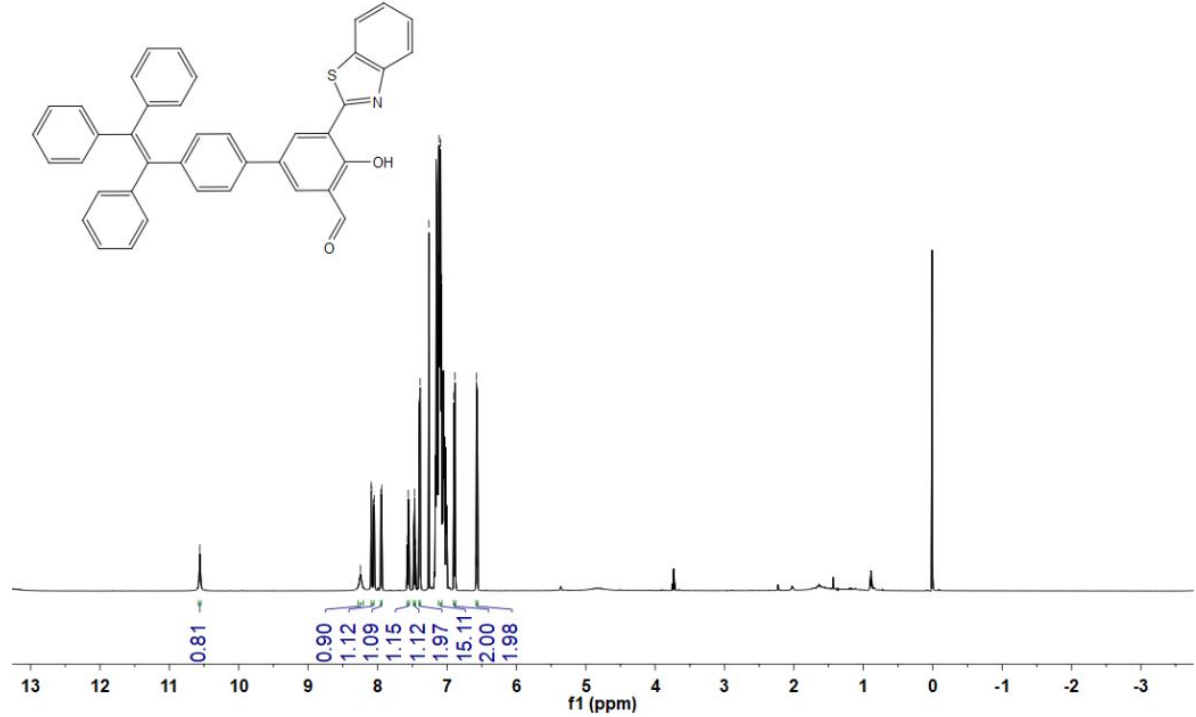

Figure S18. ${ }^{1} \mathrm{H}$ NMR spectrum of compound HBTTPEQ in $\mathrm{CDCl}_{3}$. 


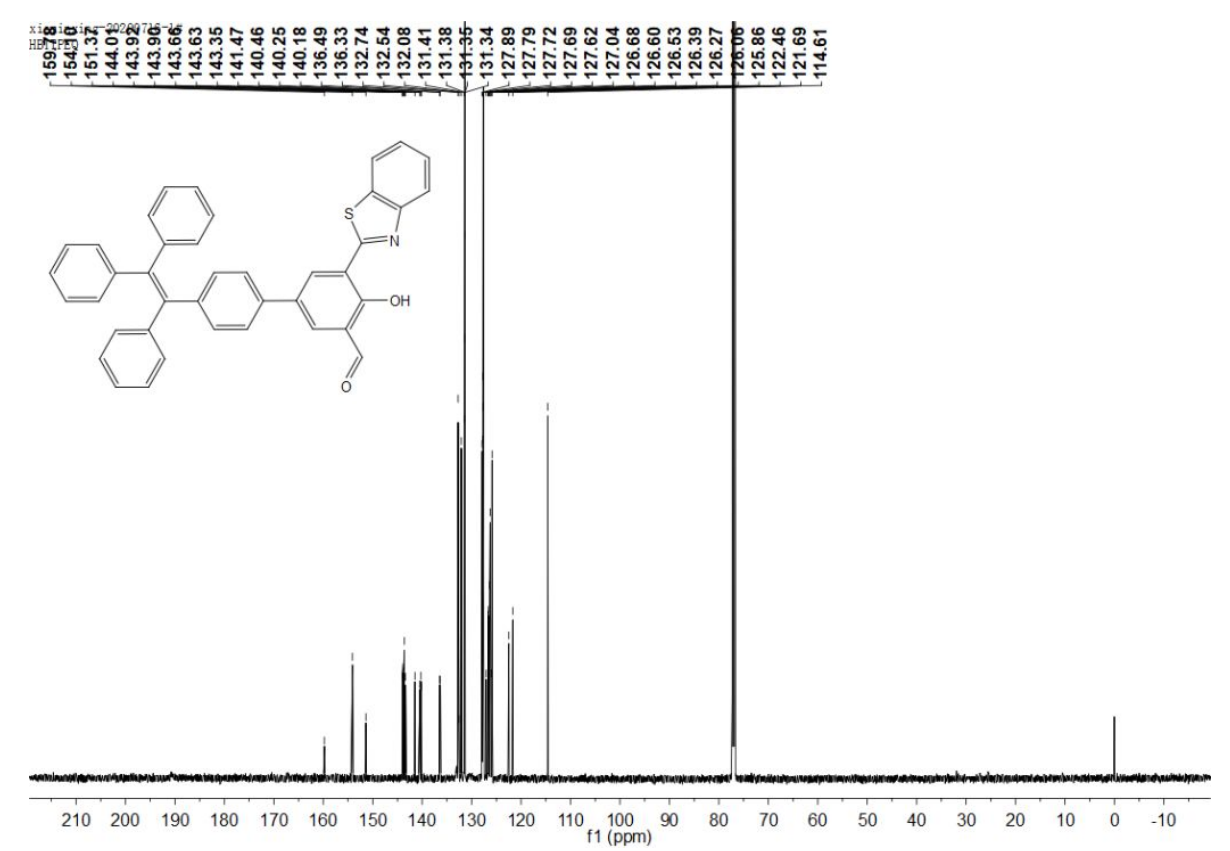

Figure S19. ${ }^{13} \mathrm{C}$ NMR spectrum of compound HBTTPEQ in $\mathrm{CDCl}_{3}$.

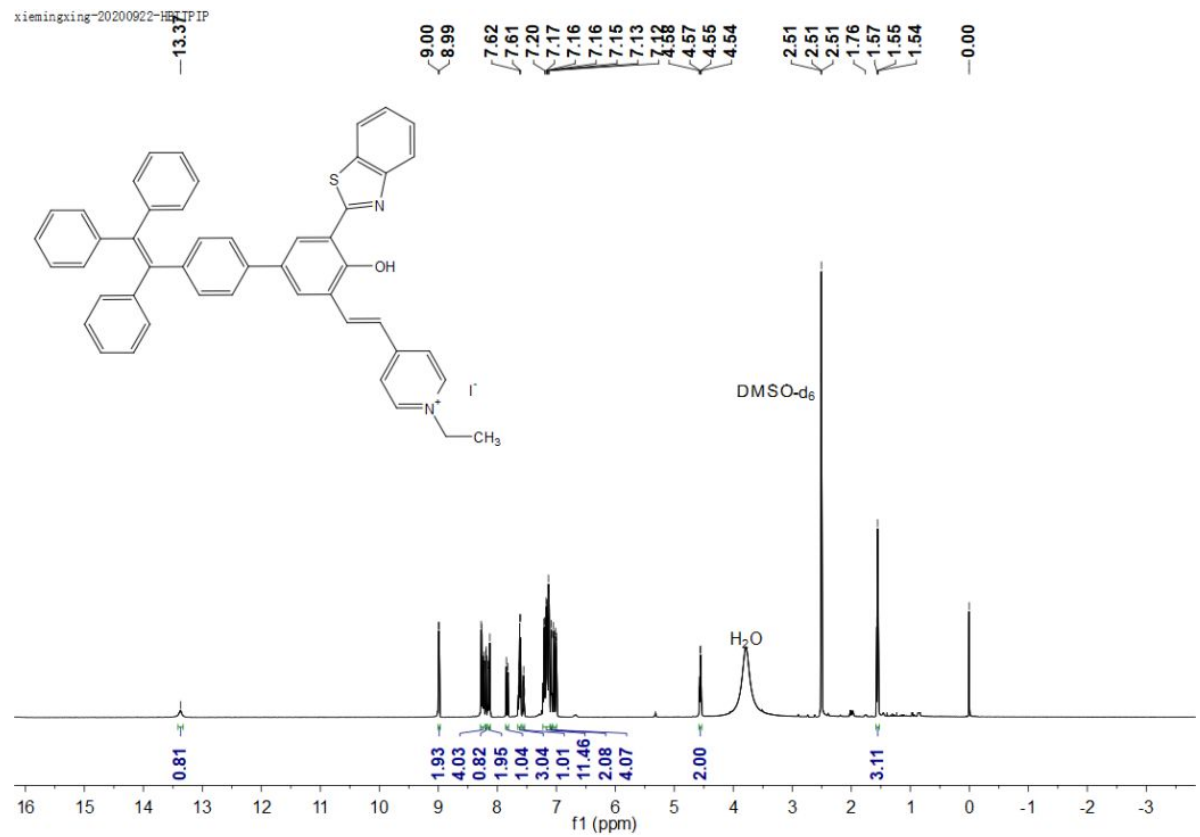

Figure S20. ${ }^{1} \mathrm{H}$ NMR spectrum of compound HBTTPEP in DMSO-d ${ }_{6}$. 


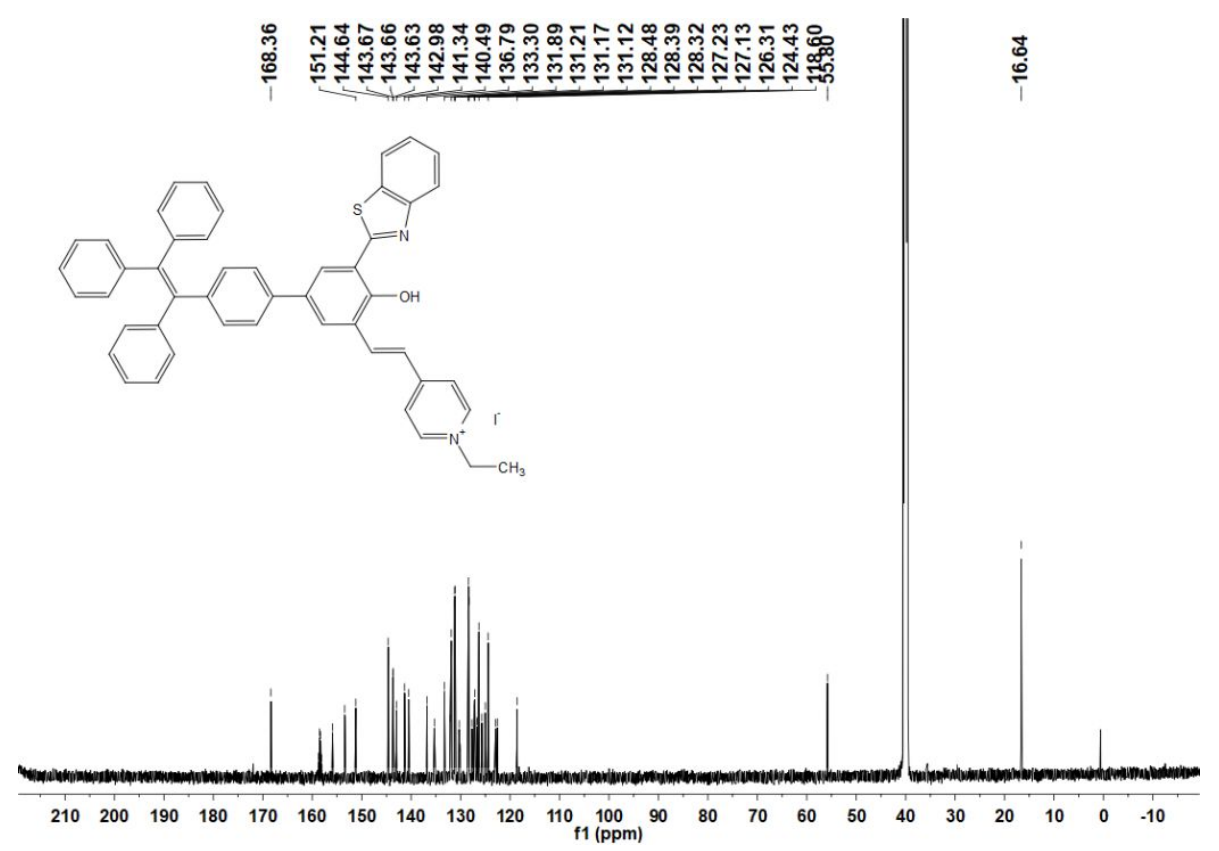

Figure S21. ${ }^{13} \mathrm{C}$ NMR spectrum of compound HBTTPEP in DMSO-d ${ }_{6}$.

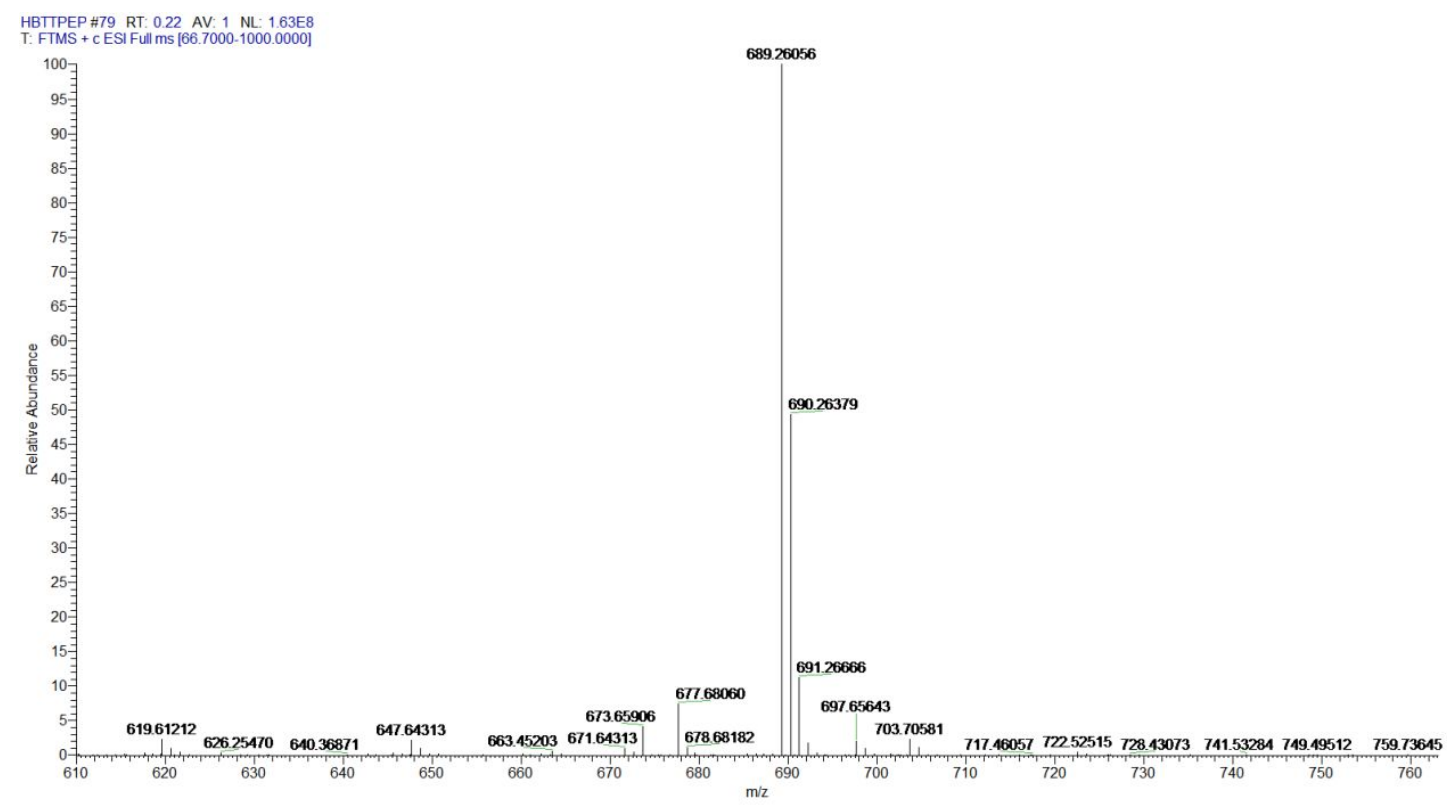

Figure S22. MS of compound HBTTPEP.
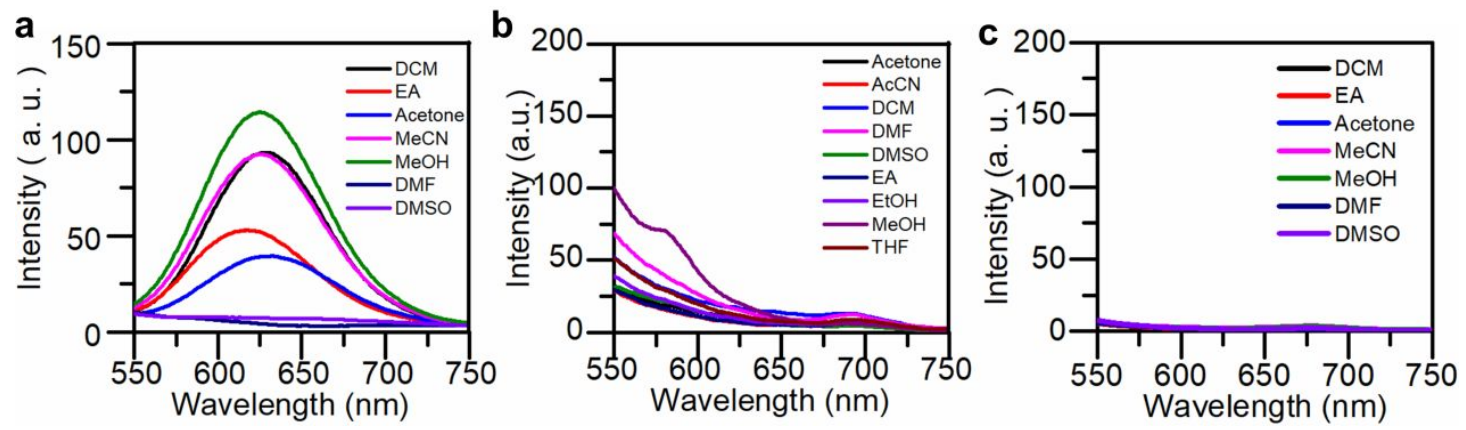

Figure S23. a-c, The emission spectra of HBTTPIP $(10 \mu \mathrm{M}), \operatorname{HBTTPEP}(10 \mu \mathrm{M})$ and 
HBTTPAP $(10 \mu \mathrm{M})$ in various solvents. (a) HBTTPIP, (b) HBTTPEP and (c) HBTTPAP.
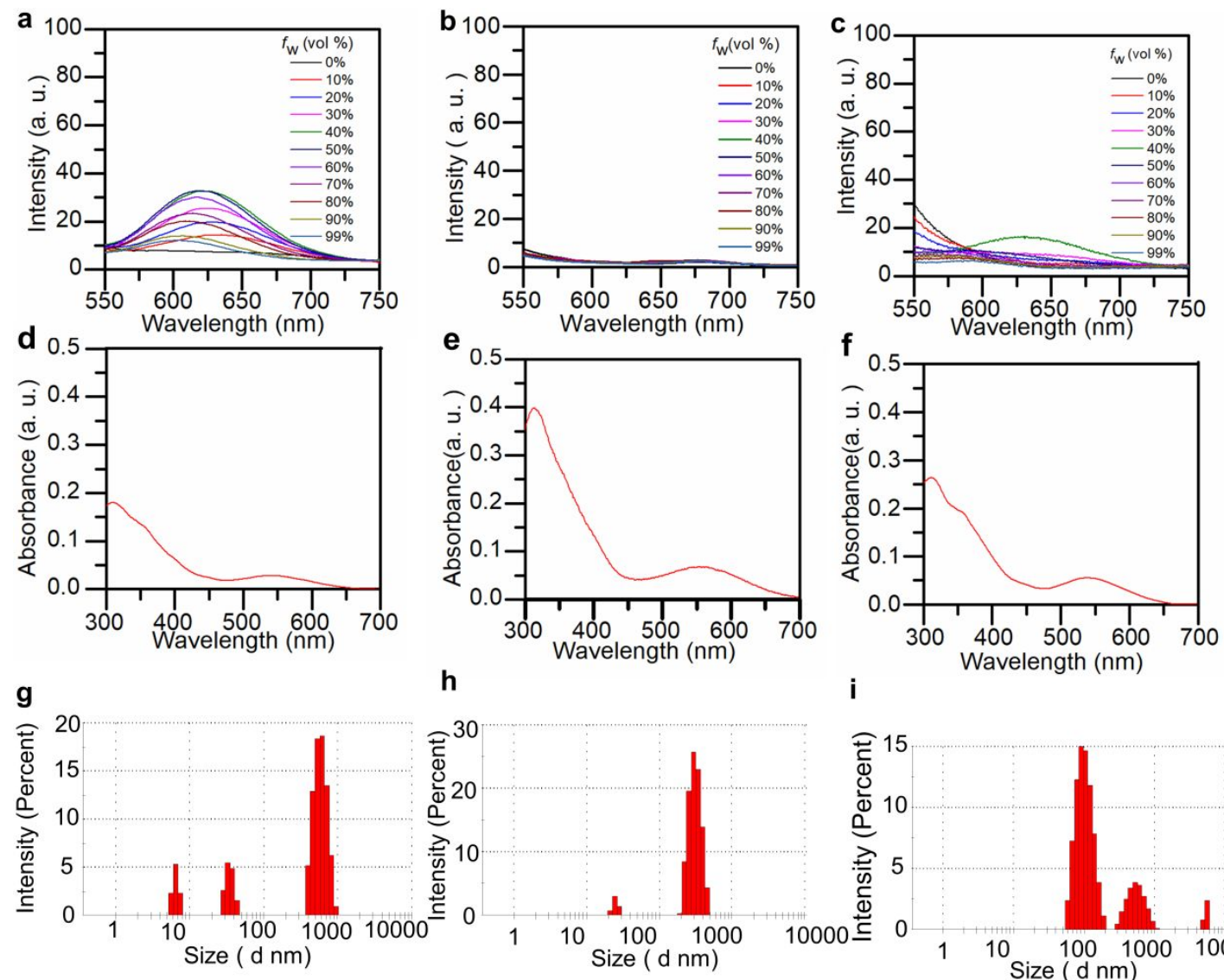

h

i

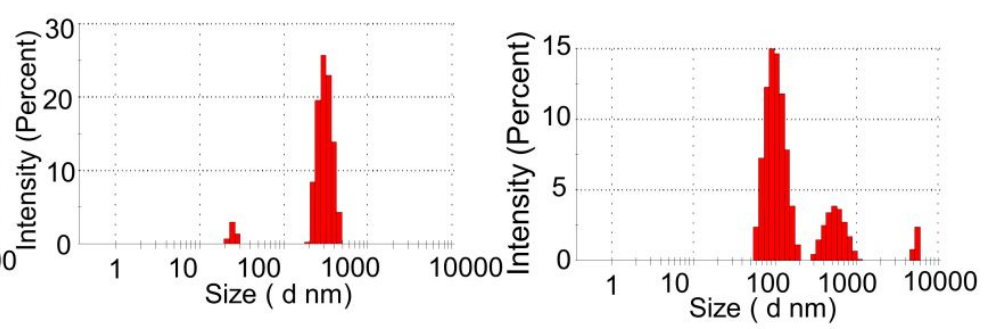

Figure S24. AIE properties of HBTTPIP, HBTTPAP and HBTTPEP with different water fractions $\left(f_{\mathrm{w}}\right)$ in DMSO. (a) Emission spectra of $\operatorname{HBTTPIP}(10 \mu \mathrm{M})$ in a mixture of DMSO-water systems $\left(\lambda_{\mathrm{ex}}=510 \mathrm{~nm}\right)$. (b) Emission spectra of $\operatorname{HBTTPAP}(10 \mu \mathrm{M})$ in a mixture of DMSO-water $\operatorname{systems}\left(\lambda_{\mathrm{ex}}=510 \mathrm{~nm}\right)$. (c) Emission spectra of $\operatorname{HBTTPEP}(10 \mu \mathrm{M})$ in a mixture of DMSO-water $\operatorname{systems}\left(\lambda_{\mathrm{ex}}=510 \mathrm{~nm}\right)$. (d) Uv-Vis Absorption spectrum of HBTTPIP $(10 \mu \mathrm{M})$ in water solution (containing 1\% DMSO). (e) Uv-Vis Absorption spectrum of HBTTPAP $(10 \mu \mathrm{M})$ in water solution (containing 1\% DMSO). (f) Uv-Vis Absorption spectrum of HBTTPEP $(10 \mu \mathrm{M})$ in water solution (containing $1 \%$ DMSO). (g) Hydrodynamic diameter of $\operatorname{HBTTPIP}(10 \mu \mathrm{M})$ in a mixture of DMSO/water(v/v=1/99). (h) Hydrodynamic diameter of HBTTPAP (10 $\mu \mathrm{M})$ in a mixture of $\mathrm{DMSO} /$ water(v/v=1/99). (i) Hydrodynamic diameter of HBTTPEP $(10 \mu \mathrm{M})$ in a mixture of DMSO/water $(\mathrm{v} / \mathrm{v}=1 / 99)$. 


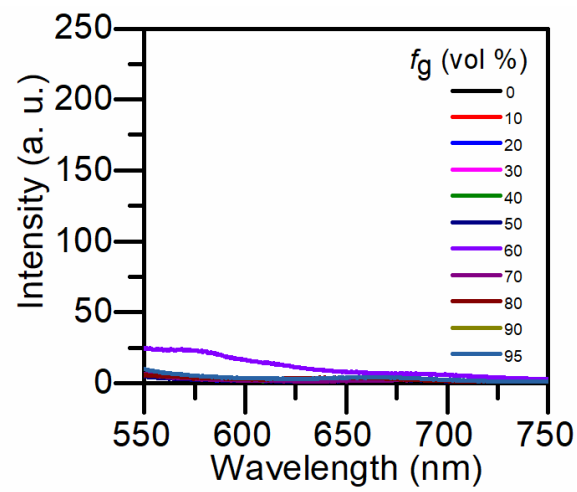

Figure S25. Emission spectra of HBTTPAP $(10 \mu \mathrm{M})$ in a mixture of water-lycerin with different glycerin fractions $\left(f_{\mathrm{g}}\right), \lambda_{\mathrm{ex}}=510 \mathrm{~nm}$.
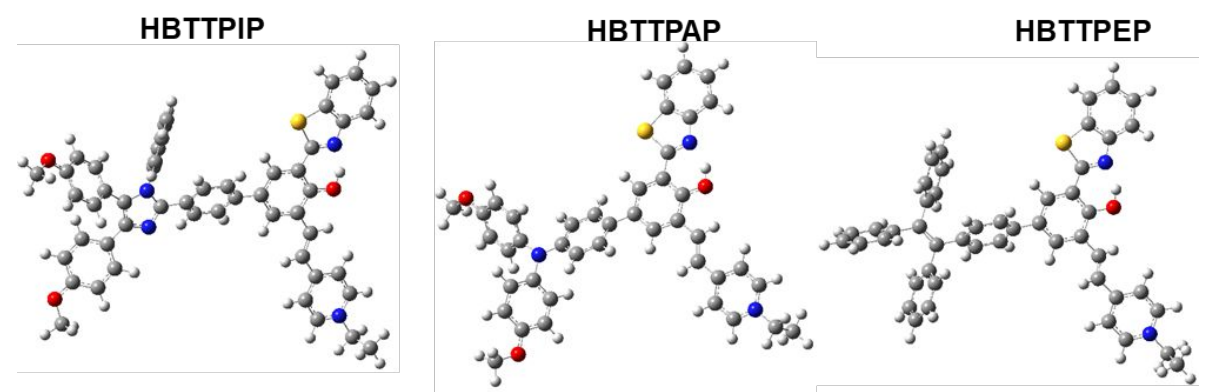

Figure S26. Optimized ground state $\left(\mathrm{S}_{0}\right)$ geometries of the molecules via DFT caculation.

Table S1. Loading efficiency (LE) of HBTTPEP in HBTTPE/GPs that were prepared using various feeding amount of HBTTPEP ( $\mathrm{n}=3$ batches $)$.

\begin{tabular}{ll}
\hline $\begin{array}{l}\text { Feeding amount of } \\
\text { HBTTPEP(mg) }\end{array}$ & $\mathrm{LE}(\%)$ \\
\hline 0.04 & $96.0 \pm 2.7$ \\
0.08 & $91.6 \pm 3.4$ \\
0.16 & $92.2 \pm 2.4$ \\
\hline
\end{tabular}


Table S2. The loading efficiency (LE) of HBTTPEP after incubation HBTTPEP with GPs $(10 \mathrm{mg} / \mathrm{mL})$ at different times.

\begin{tabular}{lc}
\hline Incubation time $(\mathrm{h})$ & $\mathrm{LE}(\%)$ \\
\hline 2 & $41.2 \pm 0.8$ \\
4 & $53.0 \pm 0.6$ \\
6 & $68.5 \pm 4.2$ \\
12 & $81.5 \pm 4.8$ \\
24 & $91.6 \pm 3.4$ \\
48 & $93.2 \pm 1.4$
\end{tabular}
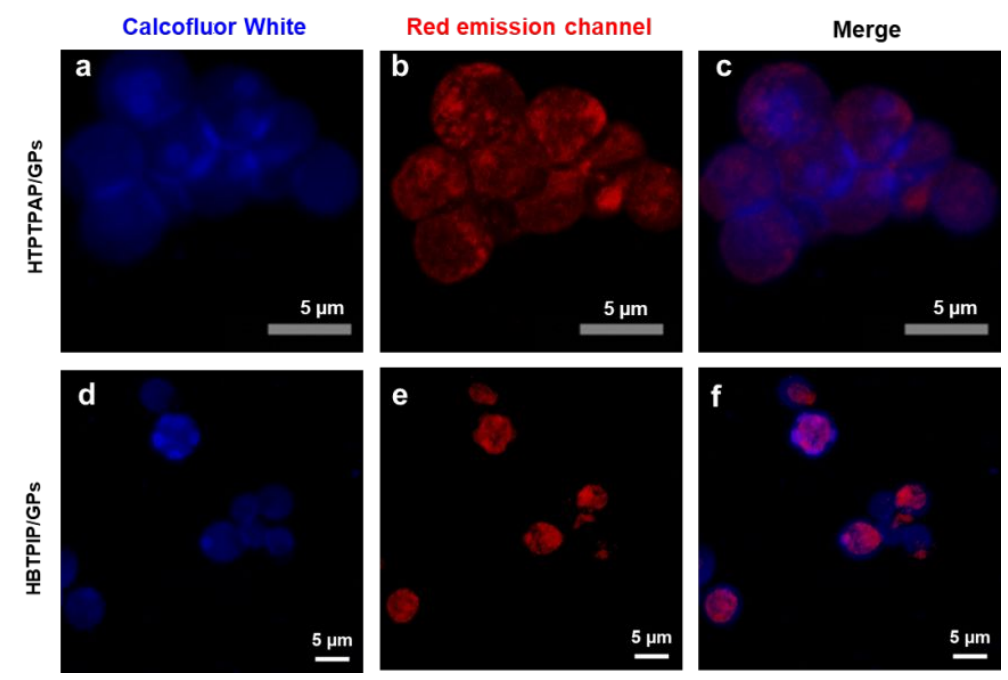

Figure S27. Fluorescent images of either Calcofluor white-stained (blue) or AIEgen-incubated GPs (red). (a and d) Calcofluor white-stained GPs. (b) HBTTPAP-incubated GPs. (e) HBTTPIP-incubated GPs. (c) Overlay of (a) and (b). (f) Overlay of (d) and (e).
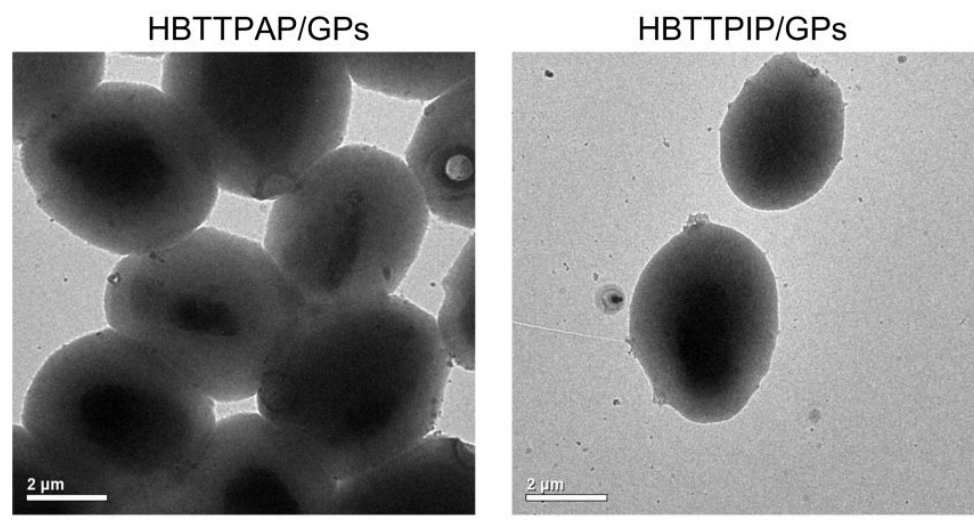

Figure S28. TEM images of HBTTPAP/GPs and HBTTPIP/GPs. 


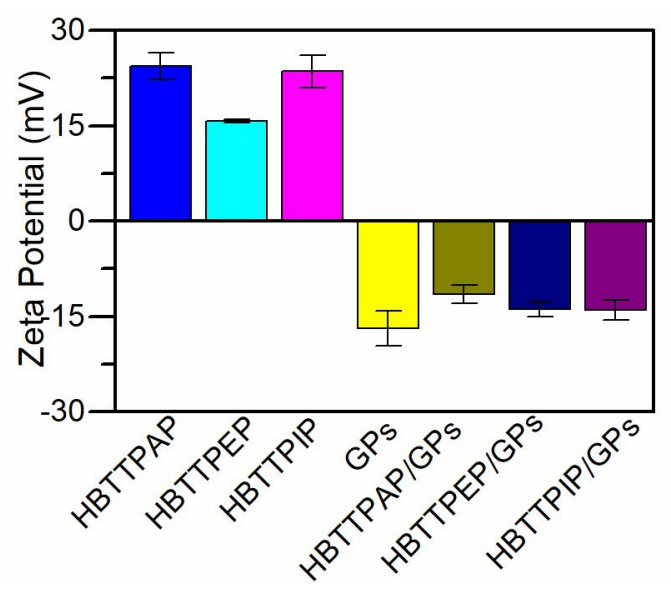

Figure S29. Zeta potential of HBTTPAP, HBTTPEP, HBTTPIP, GPs, HBTTPAP/GPs, HBTTPEP/GPs and HBTTPIP/GPs.

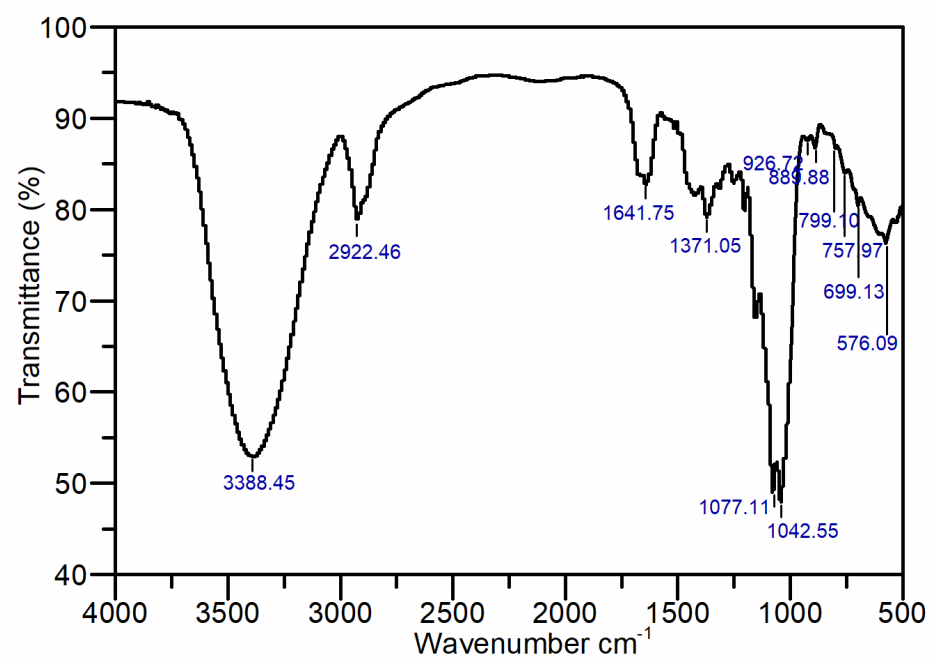

Figure S30. FT-IR spectra of the mixture of HBTTPEP and GPs.

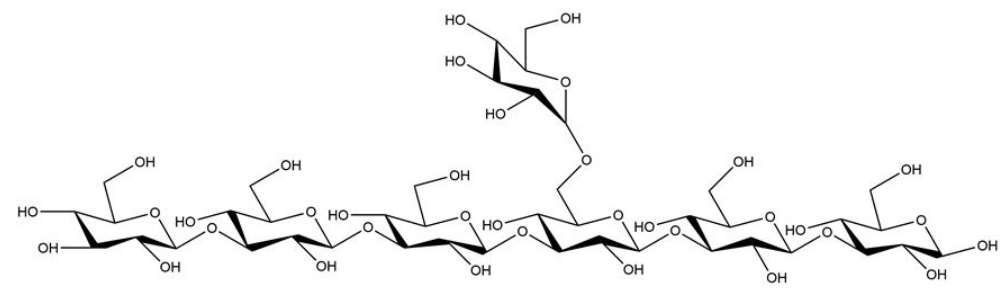

Figure S31. Repeating structural units used in the construction of the triple helix structure model of $\beta$-glucan. 

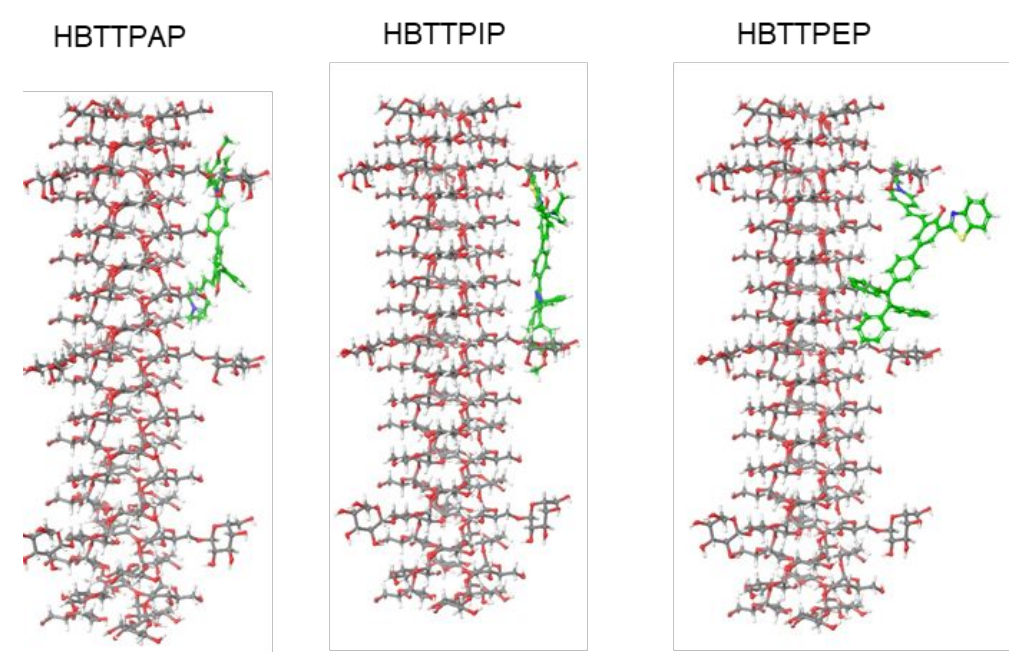

Figure S32. The results of triple helix $\beta$-glucan docking with HBTTPAP, HBTTPIP and HBTTPEP, respectively.

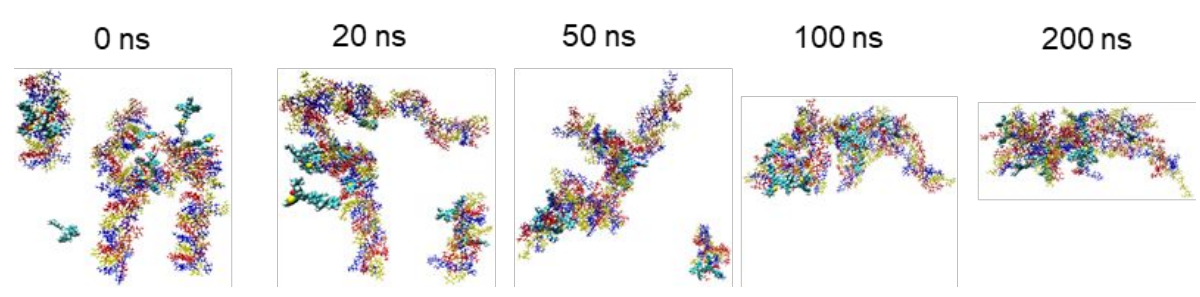

Figure S33. Sequential snapshots of MD simulations of interactions between HBTTPEP and the triple helix structure model of $\beta$-glucan. Solvent molecules water has been removed for clarity.
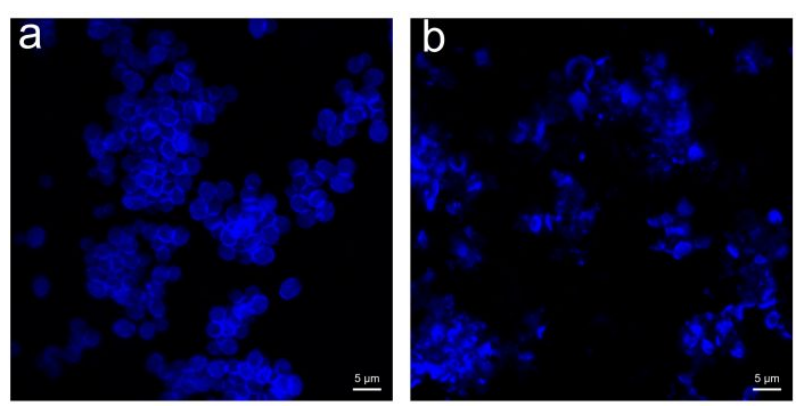

Figure S34. (a) GPs stained with calcofluor-white. (b) $\beta$-Glucan was prepared by continuously vortexing GPs with glass beads, and then stained with calcofluor-white. Scale bare $5 \mu \mathrm{m}$. 

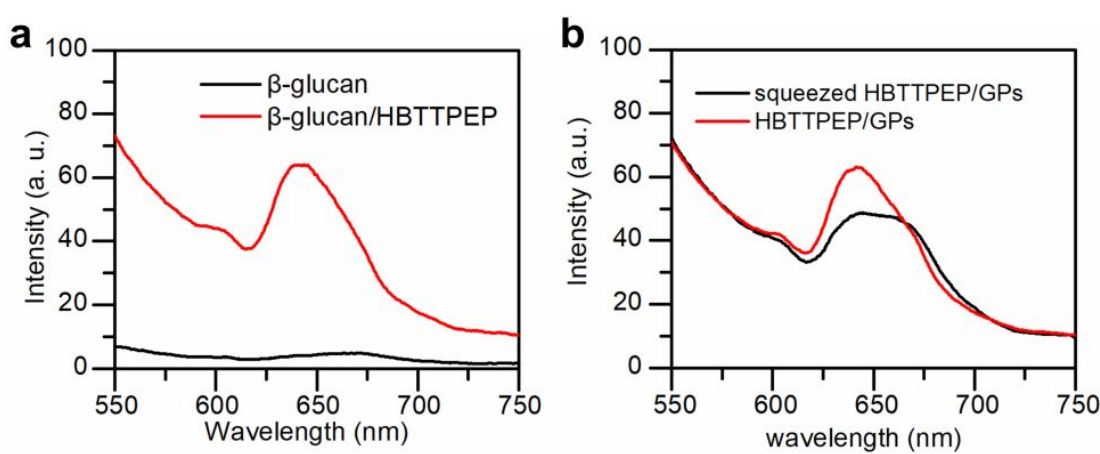

Figure S35. (a) Fluorescence emission spectrum of $\beta$-glucan in the absence and presence of HBTTPEP (b) Fluorescence emission spectrum of HBTTPEP/GPs before and after mechanical squeezing. $E_{x}=510 \mathrm{~nm}$.
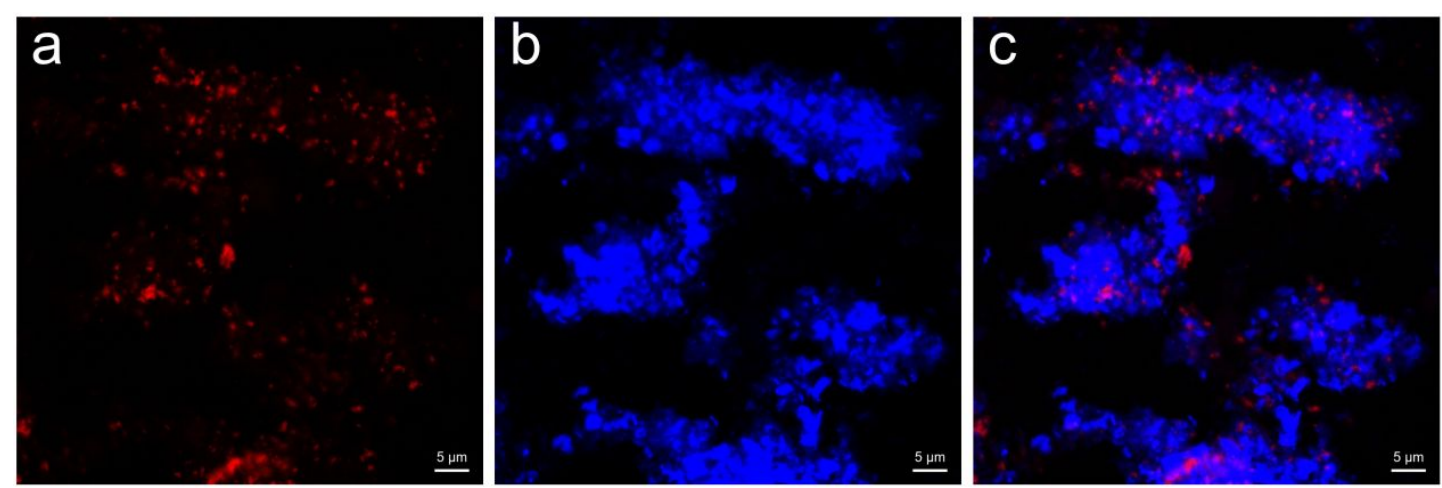

Figure S36. (a) Red emission channel of HBTTPEP/GPS after squeezing. (b) a HBTTPEP/GPS was stained with calcofluor-white after squeezing. (c) Merged image of (a) and (b). Scale bare $5 \mu \mathrm{m}$. 


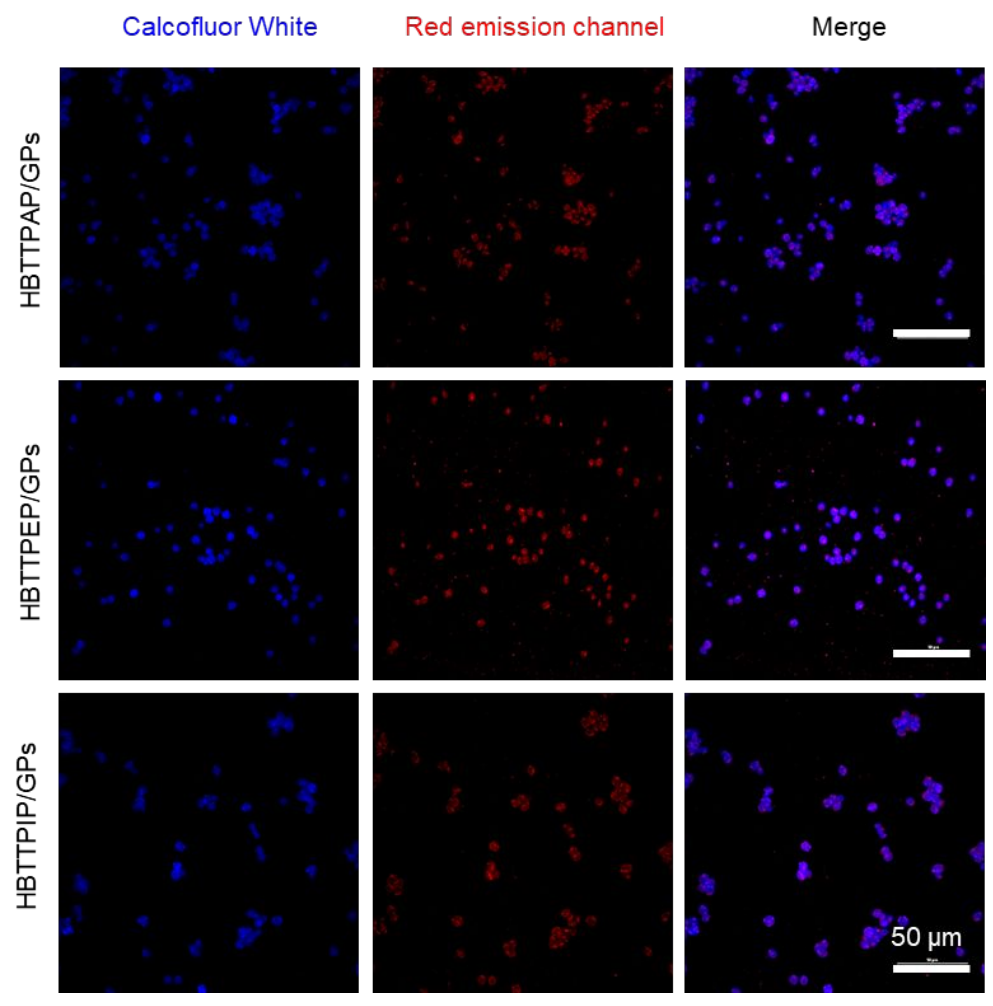

Figure S37. Fluorescent images of AIEgen/GPs after stored at $4{ }^{\circ} \mathrm{C}$ for 7 days. The cell wall of GPs was stained with Calcofluor White.
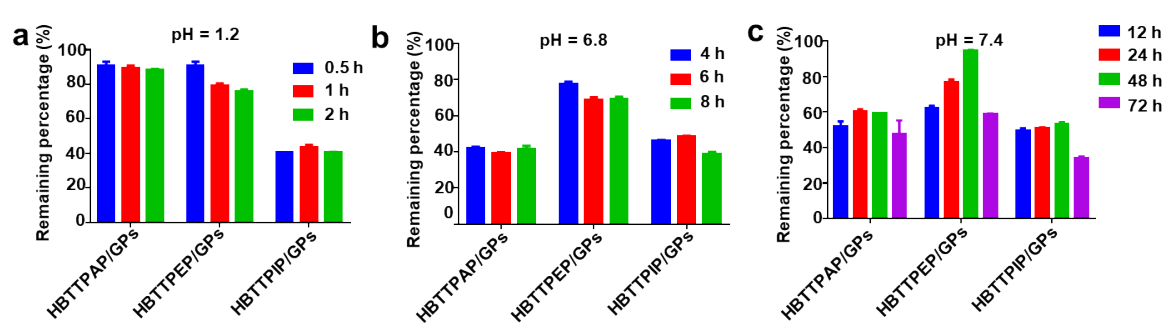

Figure S38. Stability of AIEgen/GPs in simulated gastric and intestinal fluids. (a) After sincubation AIEgen/GPs in $\mathrm{pH}=1.2$ with different times, the retention of HBTTPAP, HBTTPEP and HBTTPIP in GPs. (b) After incubation AIEgen/GPs in pH $=6.8$ with different times, the retention of HBTTPAP, HBTTPEP and HBTTPIP in GPs. (c) After incubation AIEgen/GPs in $\mathrm{pH}=7.4$ with different times, the retention of HBTTPAP, HBTTPEP and HBTTPIP in GPs. 

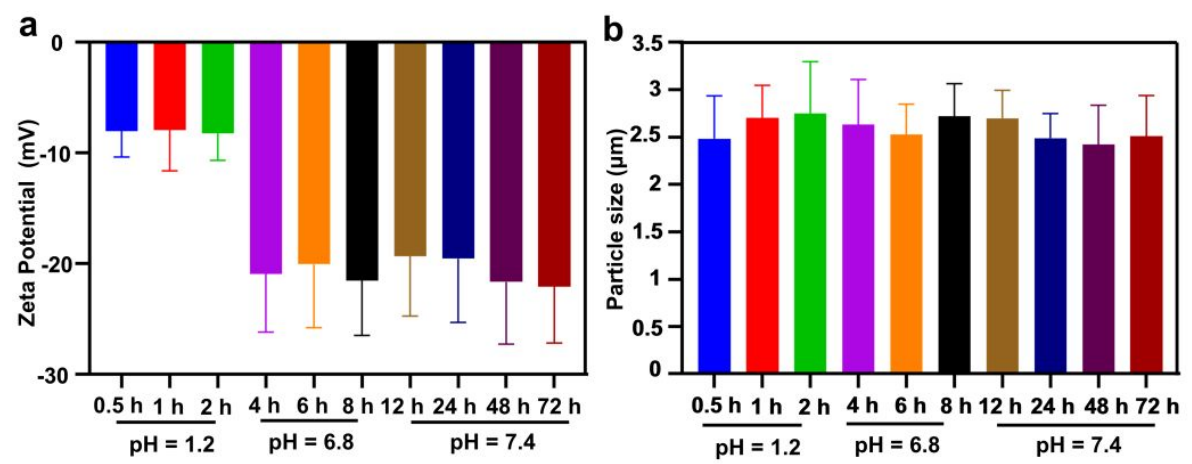

Figure S39. The Zeta potential(a) and particle size (b) of HBTTPEP/GPs after incubation for different periods of time at $\mathrm{pH}=1.2,6.8$ and 7.4.
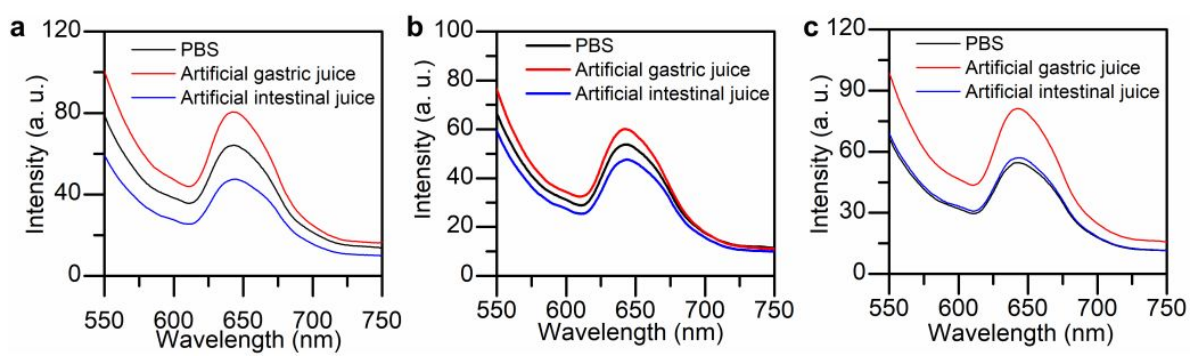

Figure S40. Fluorescence emission spectra of AIEgen/GPs in different medium.

Fluorescence emission spectra of (a) HBTTPIP/GPs, (b) HBTTPAP/GPs and (c) HBTTPEP/GPs in PBS, artificial gastric juice, and intestinal fluid, respectively. Excitation wavelength: $510 \mathrm{~nm}$. 


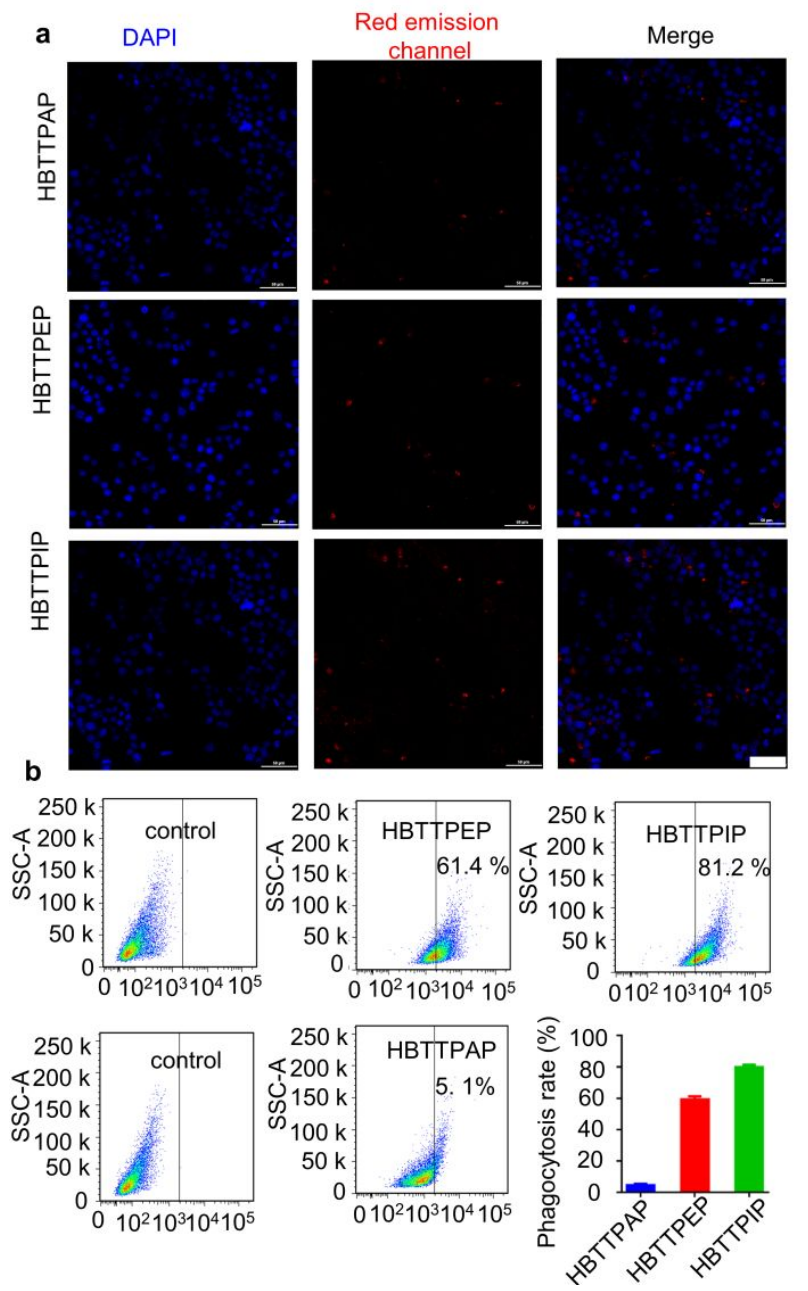

Figure S41. Cellular uptake of AIEgen in RAW264.7 (a) Confocal images of RAW264.7 cells incubated with HBTTPAP $(10 \mu \mathrm{M})$, HBTTPEP $(10 \mu \mathrm{M})$ and HBTTPIP $(10 \mu \mathrm{M})$, respectively. The nucleus was stained with DAPI. Scale bar: 50 $\mu \mathrm{m}$. (b) Flow cytometry analysis of the phagocytosis of HBTTPEP, HBTTPIP and HBTTPAP, respectively, in RAW264.7 cells. 


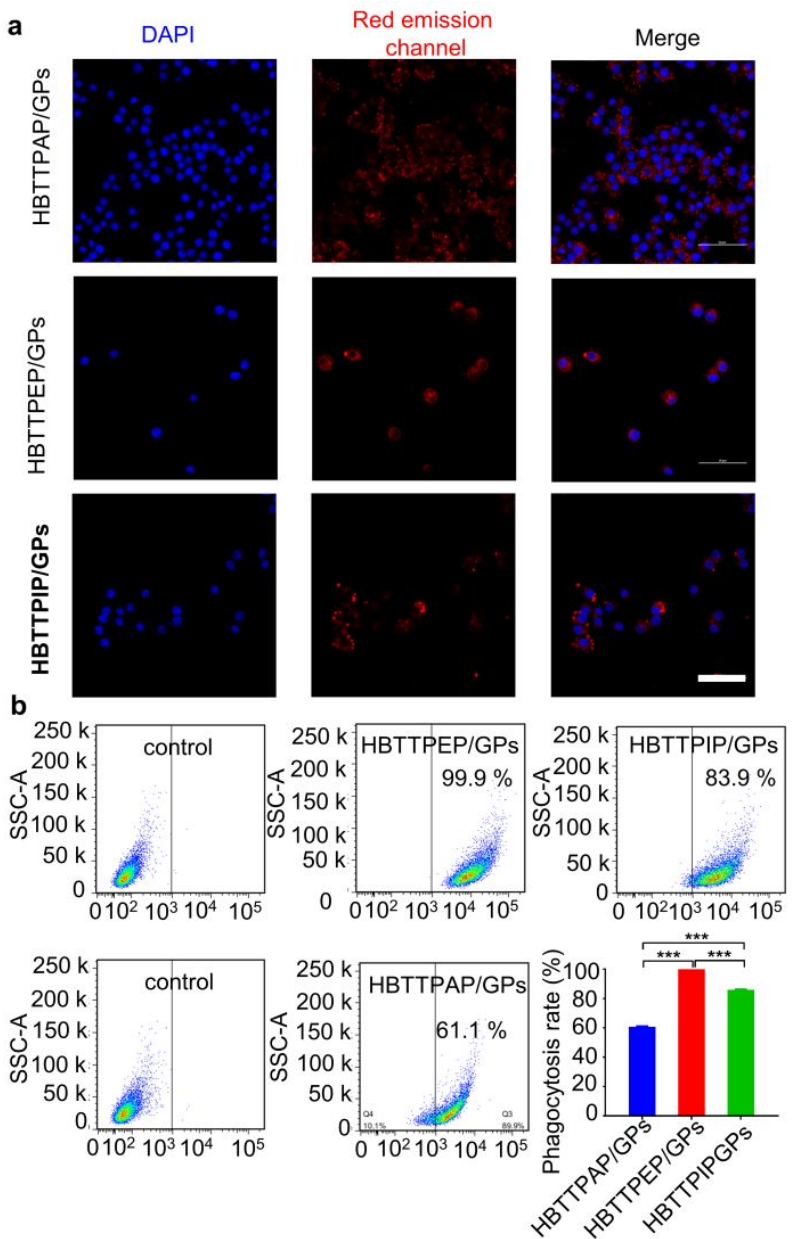

Figure S42. Cellular uptake of AIEgen/GPs in RAW264.7. (a) Confocal images of RAW264.7 cells incubated with HBTTPAP/GPs, HBTTPEP/GPs and HBTTPIP/GPs, respectively. The nucleus was stained with DAPI. Scale bar: $5 \mu \mathrm{m}$. (b) Flow cytometric analysis of macrophage phagocytosis of HBTTPAP/GPs, HBTTPEP/GPs and HBTTPIP/GPs. All bars represent mean \pm s.d. $(\mathrm{n}=3)$. $* * * \mathrm{P}<0.001$.

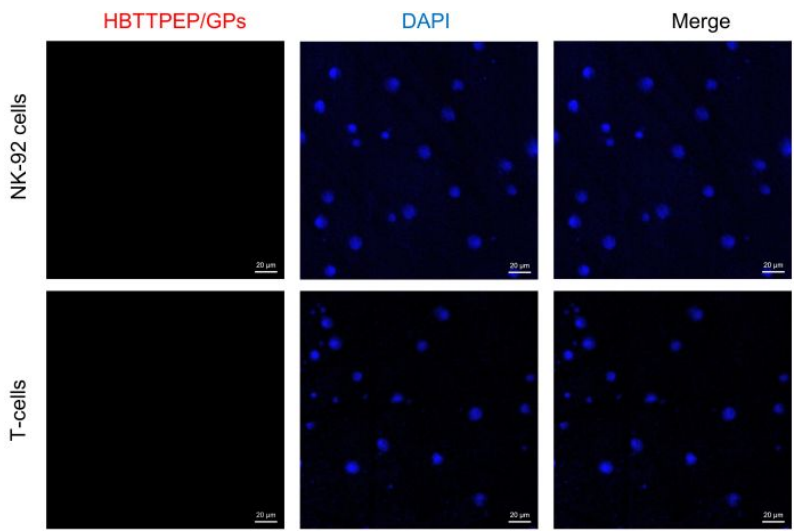

Figure S43. Confocal images of NK-92 cells and $\mathrm{T}$ cells incubated with HBTTPEP/GPs. The nucleus was stained with DAPI; Scale bar: $20 \mu \mathrm{m}$. 


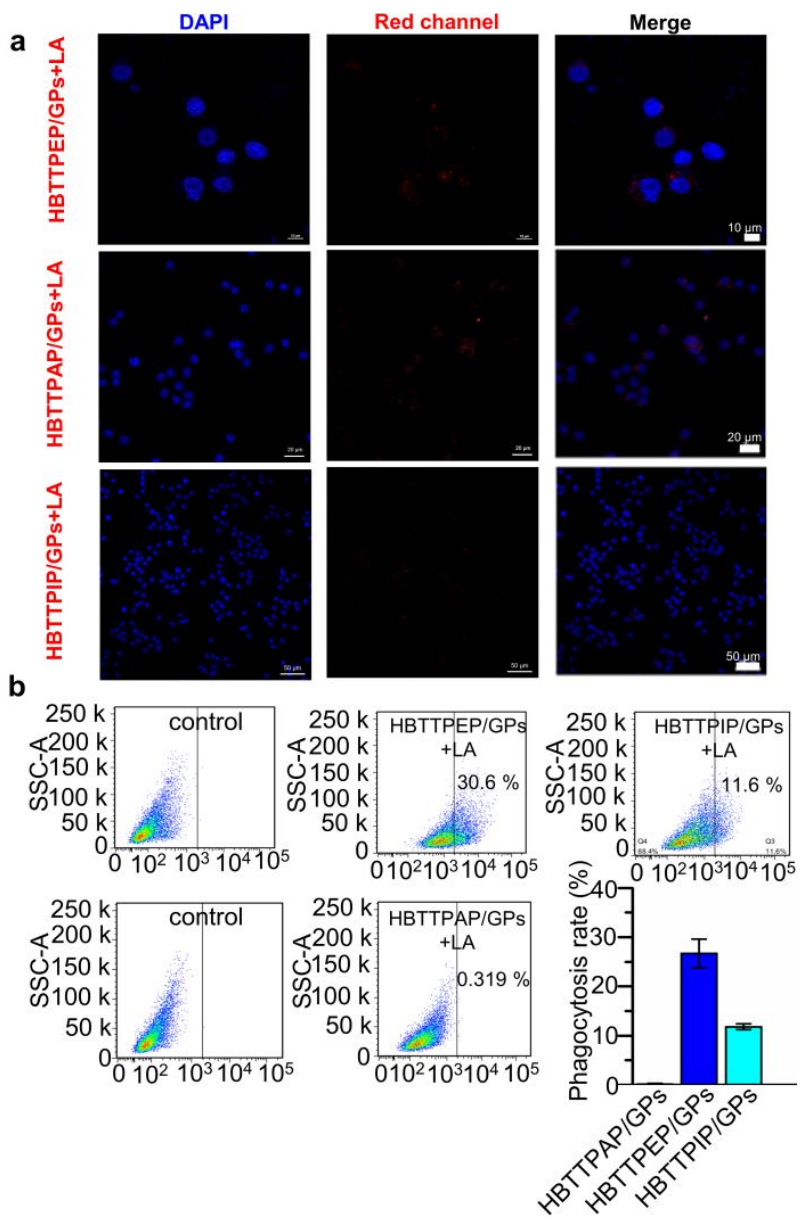

Figure S44. The mechanism of macrophages endocytosis of AIEgen/GPs. (a) Confocal fluorescence imaging to study the effect of laminarin (LA) on the uptake of HBTTPEP/GPs, HBTTPAP/GPs and HBTTPIP/GPs by macrophages. The nucleus was stained with DAPI. (b) Quantative analysis of the effect of LA on the uptake of HBTTPEP/GPs, HBTTPIPGPs and HBTTPAP/GPs by flow cytometry. All bars represent mean \pm s.d. $(n=3)$. 

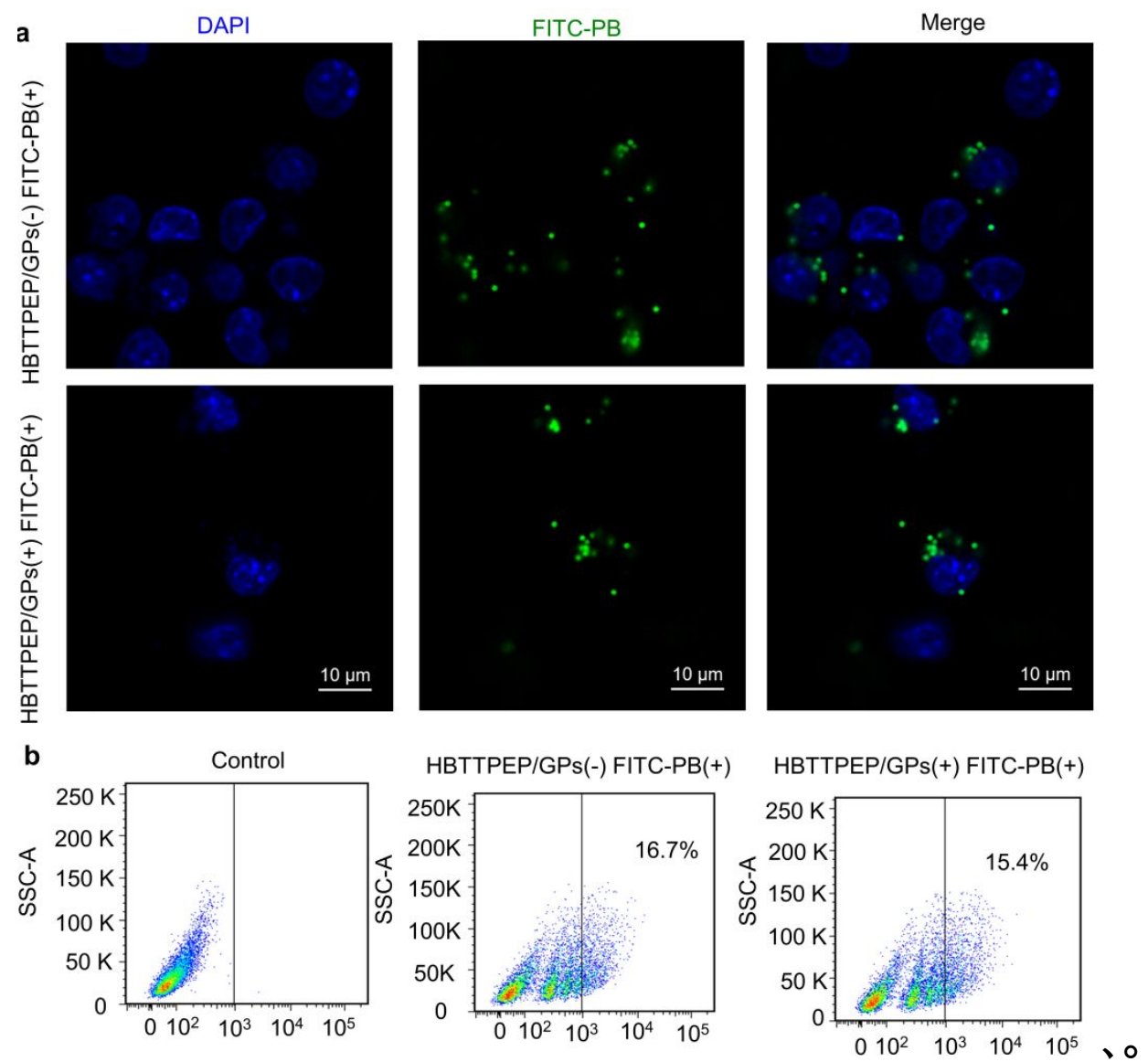

Figure S45. The effect of AIEgen/GPs on the endocytosis ability of macrophages (a) Fluorescence images of FITC-labeled polystyrene beads (FITC-PB) engulfed by macrophages RAW264.7. Macrophages were directly treated with FITC-PB (top). Macrophages were pre-treated with HBTTPEP/GPs then incubated with FITC-PB (down). (b) Flow cytometry to investigate the effect of HBTTPEP/GPs on the phagocytic function of macrophages. 

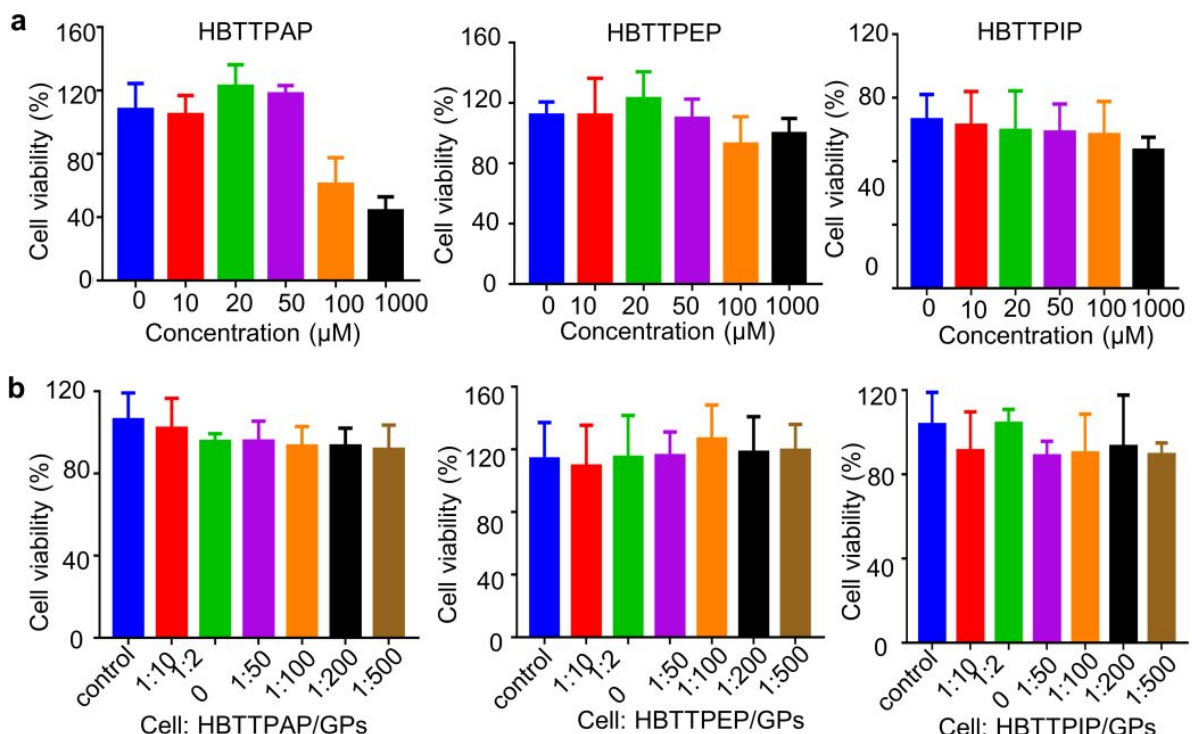

Figure S46. Effects of AIEgen and AIEgen/GPs on the proliferation activity of macrophages. (a) The effect of different concentrations of HBTTPAP, HBTTPEP and HBTTPIP on the proliferation activity of macrophages. (b) Effects of different proportions of HBTTPAP/GPs, HBTTPEP/GPs and HBTTPIP/GPs on the viability of macrophages. All bars represent mean \pm s.d. $(n=3)$.

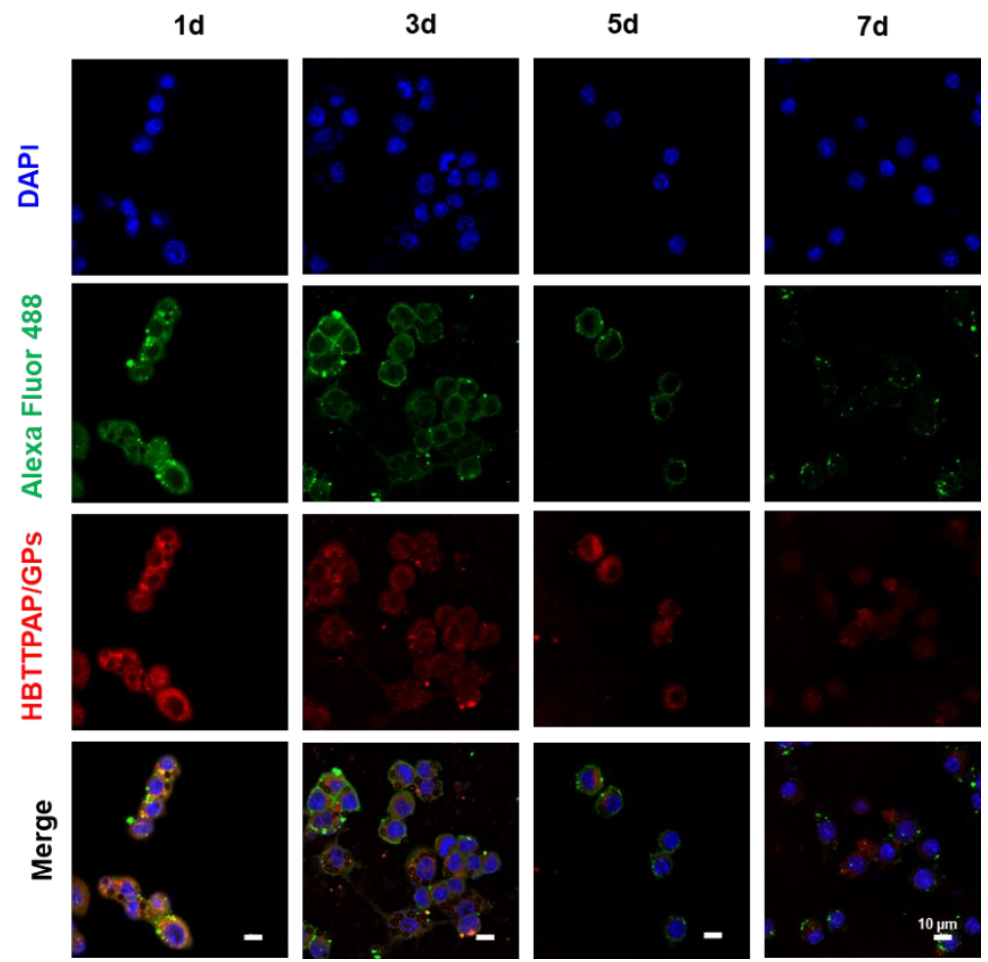

Figure S47. In vitro long term of tracking RAW264.7 with HBTTPAP/GPs. Nuclei were stained with DAPI (blue). Alexa Fluor 488 dye (green) was used for imaging the microtubule cytoskeleton in RAW264.7 cells. 


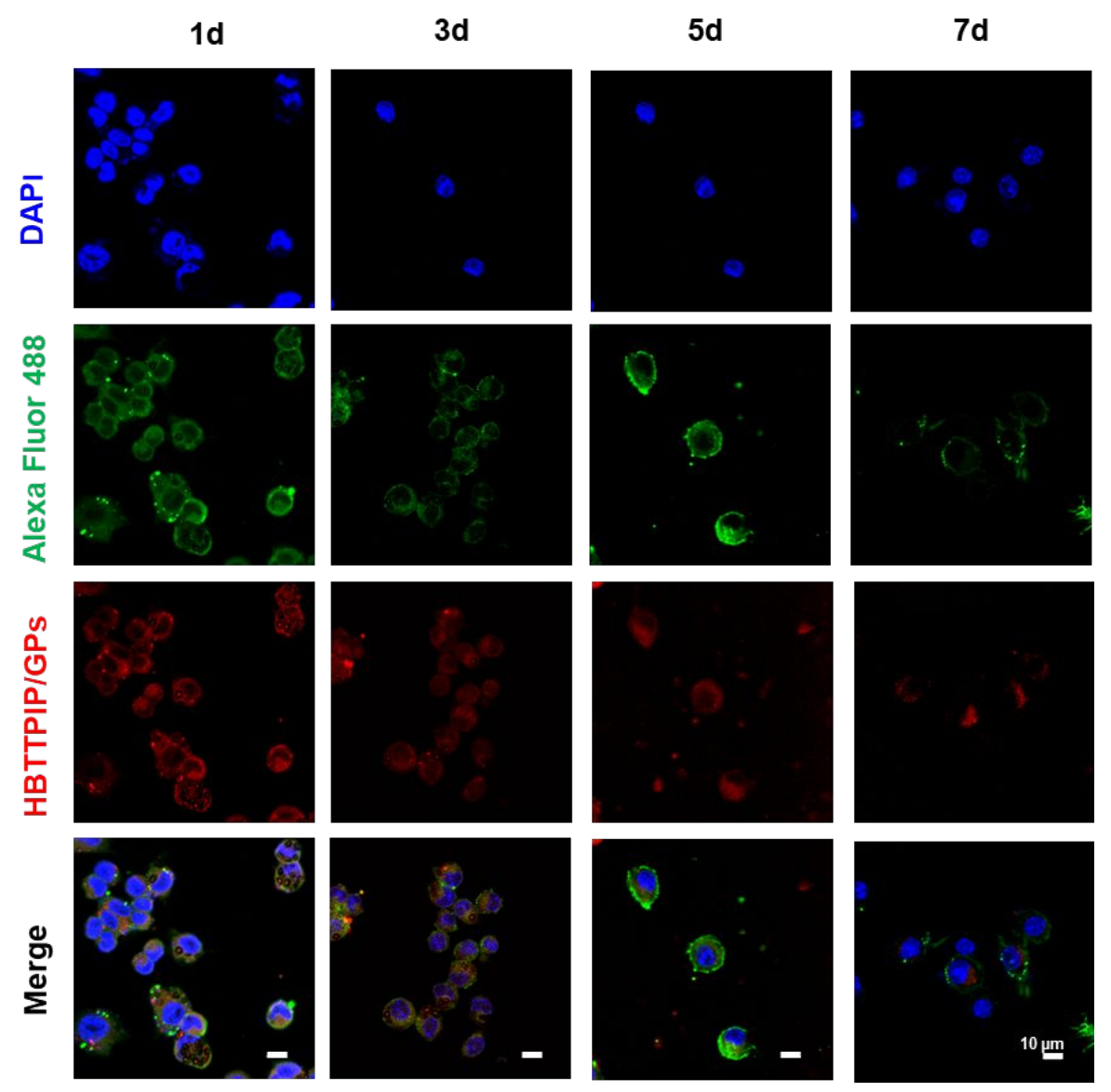

Figure S48. In vitro long term of tracking RAW264.7 with HBTTPIP/GPs. Nuclei were stained with DAPI (blue). Alexa Fluor 488 dye (green) was used for imaging the microtubule cytoskeleton in RAW264.7 cells. 
Table S3. Hematology parameters in whole blood of healthy mice, allogeneic transplanted mice and syngeneic transplanted mice following oral administration of HBTTPEP/GPs for $24 \mathrm{~h}$. All bars represent mean \pm s.d. $(\mathrm{n}=3)$.

\begin{tabular}{|l|l|l|l|l|}
\hline Hematologic Parameters & Control & Allograft & Isograft & Reference \\
\hline White blood cell $\left(10^{9} / \mathrm{L}\right)$ & $4.3 \pm 0.3$ & $2.4 \pm 0.8$ & $5.1 \pm 0.8$ & $0.8-6.8$ \\
Lymphocytes $\left(10^{9} / \mathrm{L}\right)$ & $2.3 \pm 0.2$ & $1.4 \pm 0.6$ & $3.6 \pm 1.5$ & $0.7-5.7$ \\
Monocytes $\left(10^{9} / \mathrm{L}\right)$ & $0.3 \pm 0.2$ & $0.13 \pm 0.05$ & $0.26 \pm 0.06$ & $0.0-0.3$ \\
Neutrophil(10\%/L) & $1.7 \pm 0.1$ & $0.8 \pm 0.3$ & $1.2 \pm 0.6$ & $0.1-1.8$ \\
Lymphocytes (\%) & $53.4 \pm 4.7$ & $60.4 \pm 7.1$ & $67.9 \pm 18.05$ & $55.8-90.6$ \\
Red blood cell (10/2/L) & $6.2 \pm 2.0$ & $9.2 \pm 0.2$ & $8.6 \pm 0.5$ & $6.36-9.42$ \\
Hemoglobin (g/L) & $96.3 \pm 3.1$ & $130 \pm 4.6$ & $126.3 \pm 6.1$ & $110-143$ \\
Hematocrit (\%) & $28.6 \pm 9.8$ & $44.9 \pm 1.02$ & $42.7 \pm 2.3$ & $34.6-44.6$ \\
Mean corpuscular volume (fl) & $46.0 \pm 1.1$ & $48.9 \pm 0.6$ & $49.9 \pm 0.5$ & $48.2-58.3$ \\
Mean corpuscular hemoglobin (pg) & $17.0 \pm 6.9$ & $14.1 \pm 0.2$ & $14.7 \pm 0.2$ & $15.8-19$ \\
Mean corpuscular hemoglobin concentration & $280.0 \pm 2.8$ & $288.7 \pm 5.1$ & $295.3 \pm 4.2$ & $302-353$ \\
(g/L) & $16.6 \pm 0.54$ & $15.6 \pm 0.2$ & $14.3 \pm 0.0 .7$ & $13-17$ \\
Red blood cell distribution width (\%) & & & \\
\hline
\end{tabular}
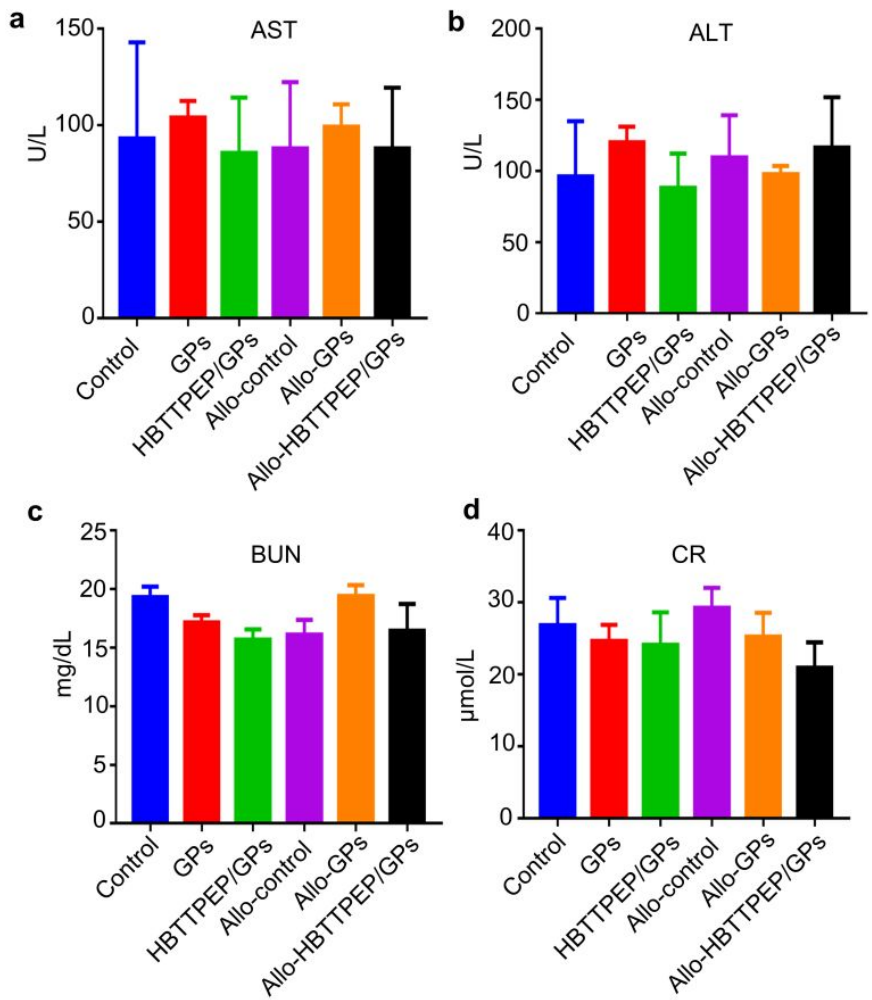

Figure S49. In vivo liver and kidney functional markers examined in the peripheral blood of healthy mice and allograft mice. Serum levels of (a) aspartate transaminase 
(AST); (b) alanine aminotransferase (ALT); (c) urea nitrogen (BUN); (d) creatinine (CR). All bars represent mean \pm s.d. $(n=3)$.
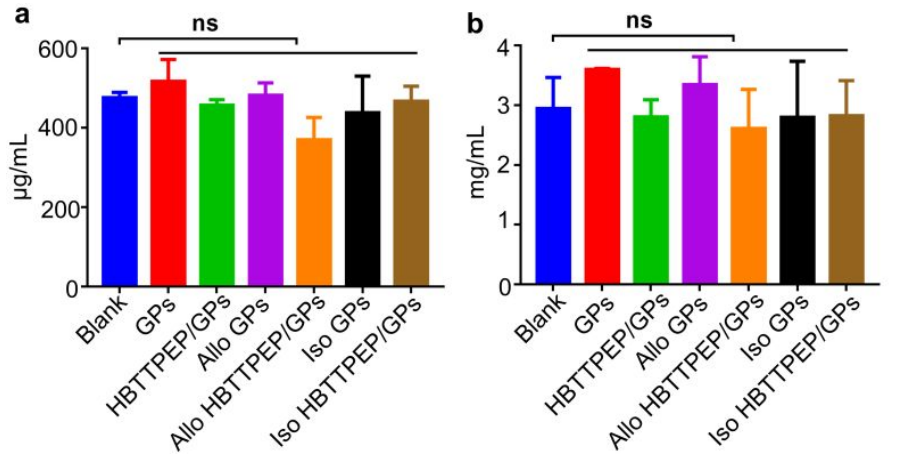

Figure S50. Immunogenicity of GPs and HBTTPEP/GPs. Allograft mice (POD 6), isograft mice (POD 6) and healthy mice were orally administrated HBTTPEP/GPs or GPs. The healthy mice were used as blank control. The level of $\operatorname{IgM}(\mathrm{a})$ and $\operatorname{IgG}(\mathrm{b})$ in serum were measured 24 hours later. All bars represent mean \pm s.d. $(n=3)$, ns $=$ no significance.
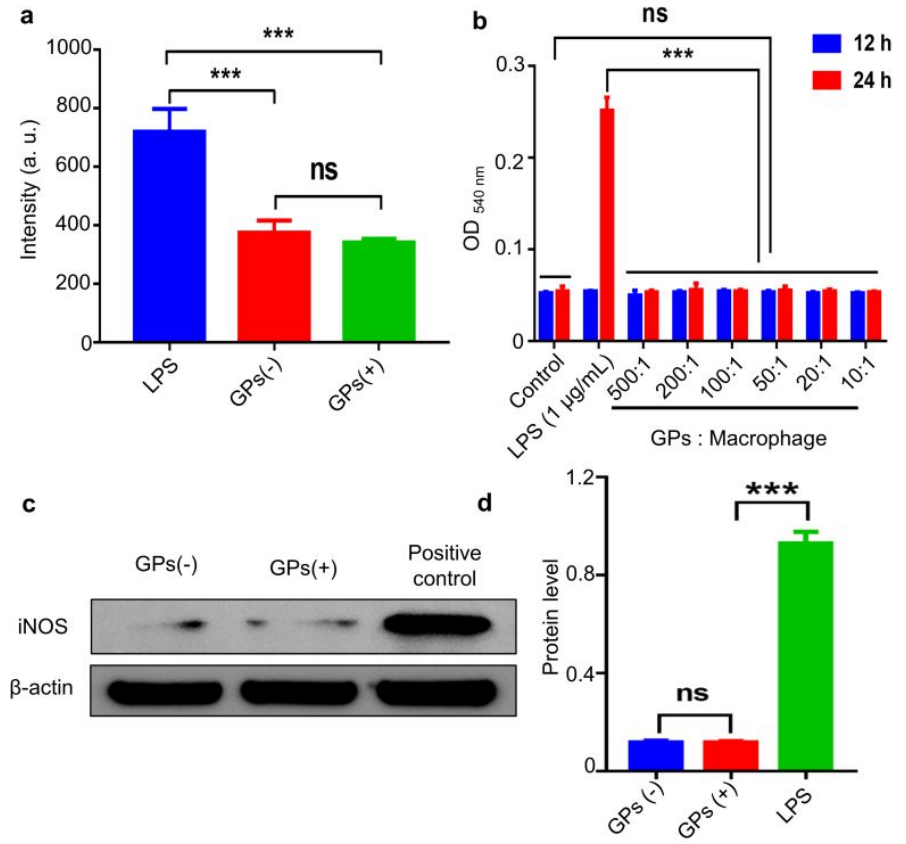

Figure S51. Immune response of macrophage treated with GPs. (a) Detection of ROS generation by DCFH-DA in RAW 264.7 cells after incubation with LPS, PBS and GPS, respectively. (b) Detection of NO generation by nitric oxide Assay Kit in RAW 264.7 cells after incubation with PBS, LPS, and GPS, (c) Effect of GPs on expression of iNOS, RAW264.7 cells were pretreated with GPs. Protein was detected by western blotting. (d) Protein expression was quantitated by densitometric analysis of western 
blot. All data are represented as mean \pm SD from three independent experiments. $* * * \mathrm{P}<0.001 ; \mathrm{ns}=$ no significance.
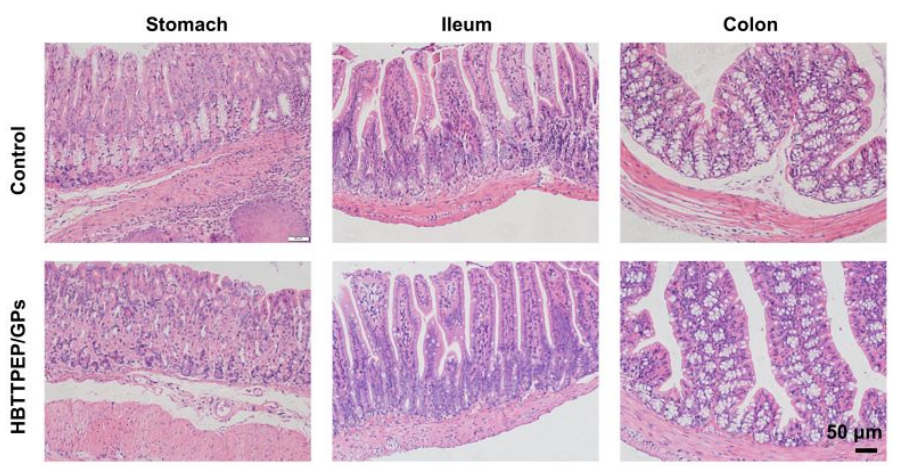

Figure S52. H\&E-stained images of stomach, ileum, and colon tissue sections.

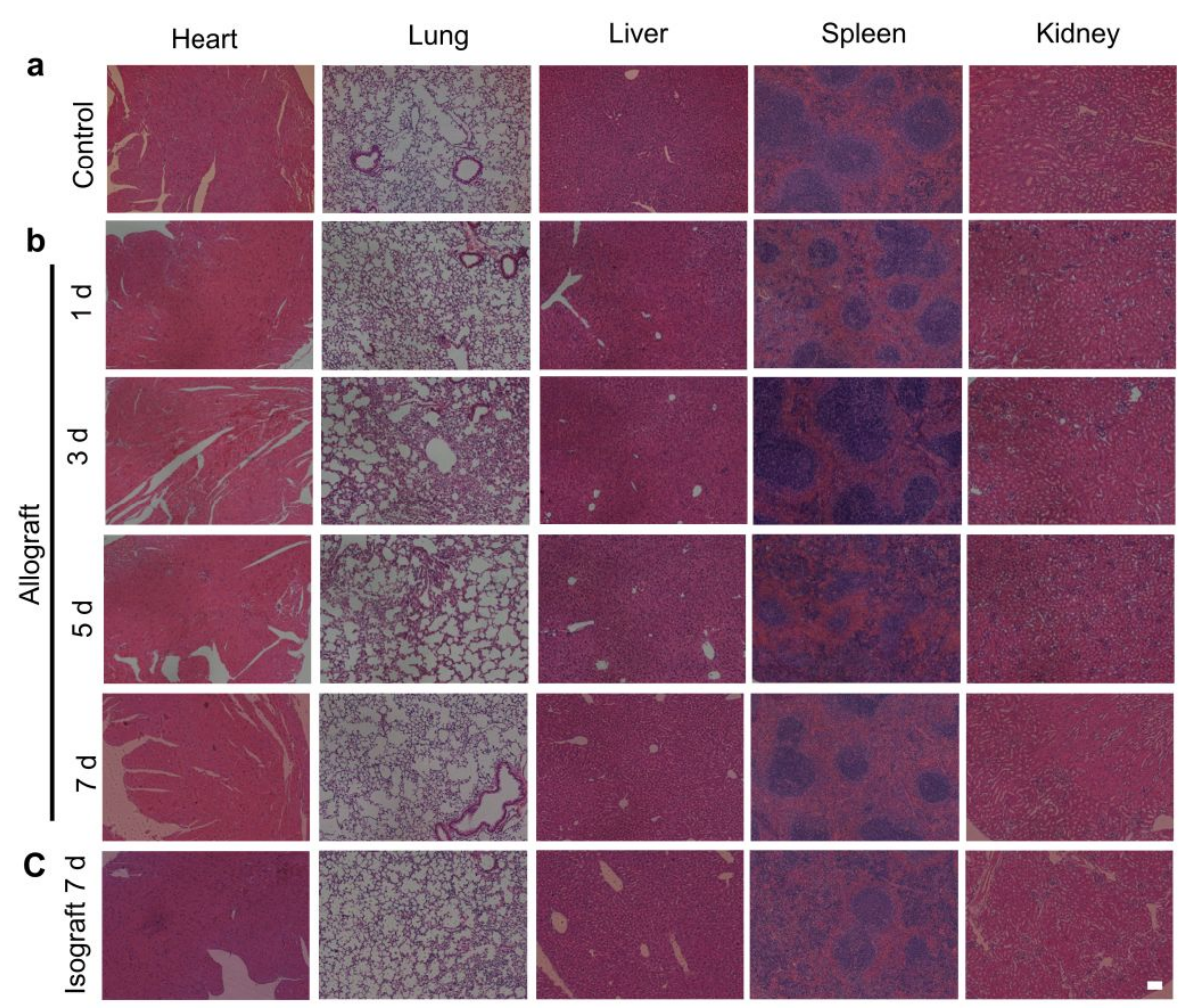

Figure S53. H\&E staining of major organs such as heart, lungs, liver, spleen, and kidneys. (a) H\&E-staining of major organs from healthy mice. (b) H\&E staining of main organ sections after oral administration of HBTTPEP/GPs in allogeneic transplanted mice for 1, 3, 5 and 7 days. (c) H\&E staining of main organ sections after oral administration of HBTTPEP/GPs in isograft mice for 7 days. Scale bare: $100 \mu \mathrm{m}$. 

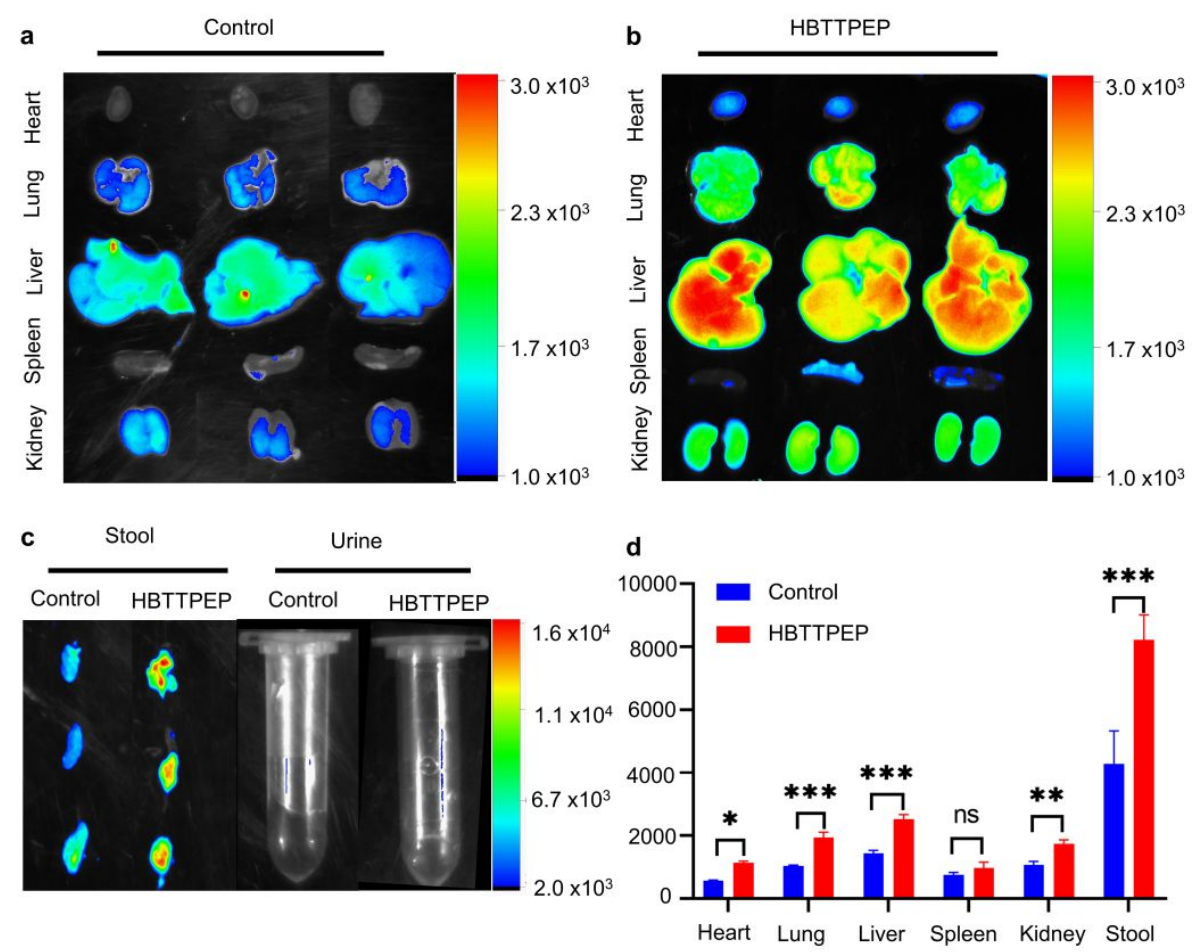

Figure S54. In vivo biodistribution after oral HBTTPEP $(0.8 \mathrm{mg} / \mathrm{kg})$. (a) Fluorescence imaging of the heart, liver, spleen, lung and kidney of allogeneic transplanted mice. (b) Fluorescence imaging of heart, liver, spleen, lung and kidney of allogeneic transplanted mice after oral administration of HBTTPEP for $24 \mathrm{~h}$. (c) Fluorescence imaging of feces and urine of mice in the control and experimental groups (d) Quantitative analysis of fluorescence signal in various organs. $E x=510 \mathrm{~nm} ; E_{\mathrm{em}}=700$ nm. $* \mathrm{P}<0.05, * * \mathrm{P}<0.01, * * * \mathrm{P}<0.001, \mathrm{~ns}=$ no significance.

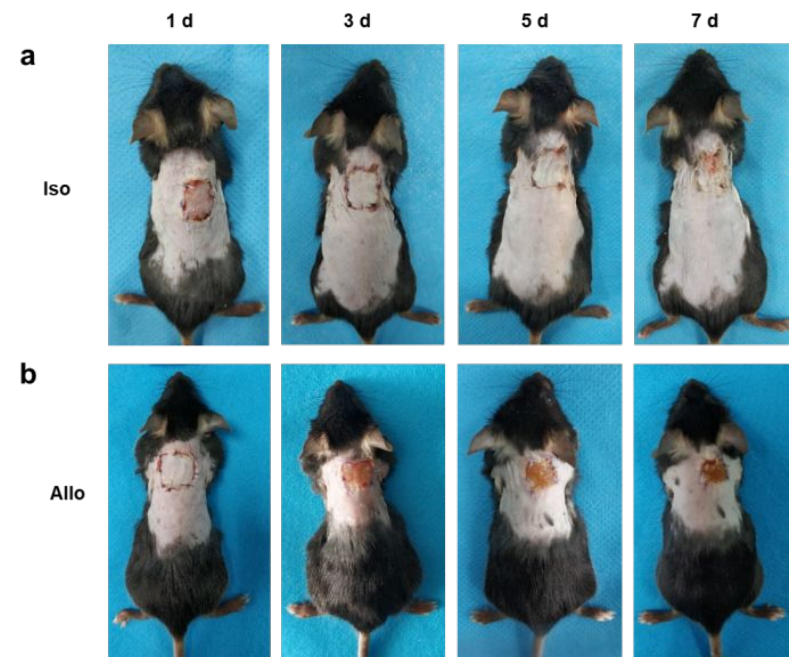

Figure S55. Photograph of allograft and isograft mice. (a) Representative pictures of $1,3,5$, and 7 days after of syngeneic transplantation. (a) Representative pictures of 1 , 
3,5 , and 7 days after of allogeneic transplantation.

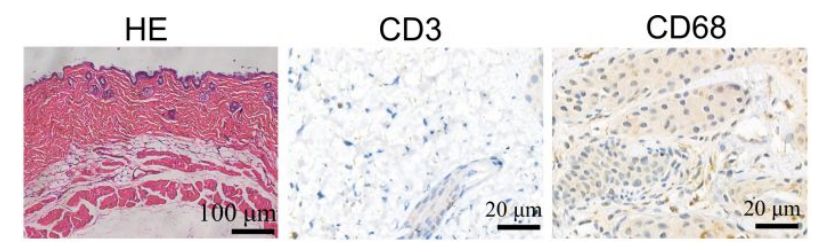

Figure S56. Hematoxylin and eosin staining, CD3 immunohistochemistry and CD68 immunohistochemistry analysis of skin from healthy mouse.

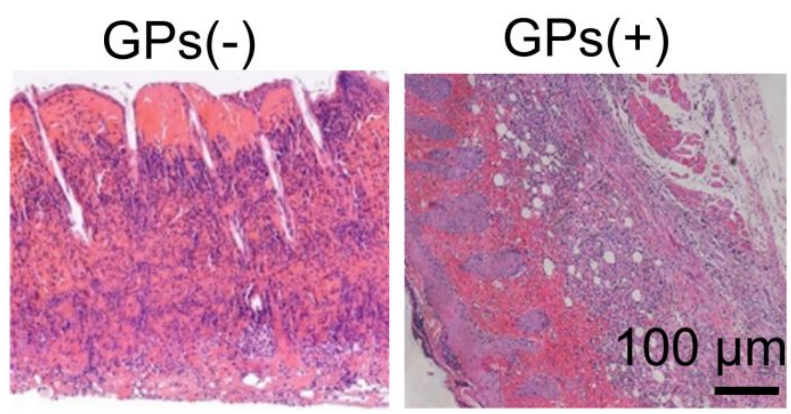

Figure S57. Histologic analysis of grafted skin after 7 days transplantation. GPs(-): allograft mice orally administered PBS; GPs $(+)$ :allograft mice orally administered GPs.

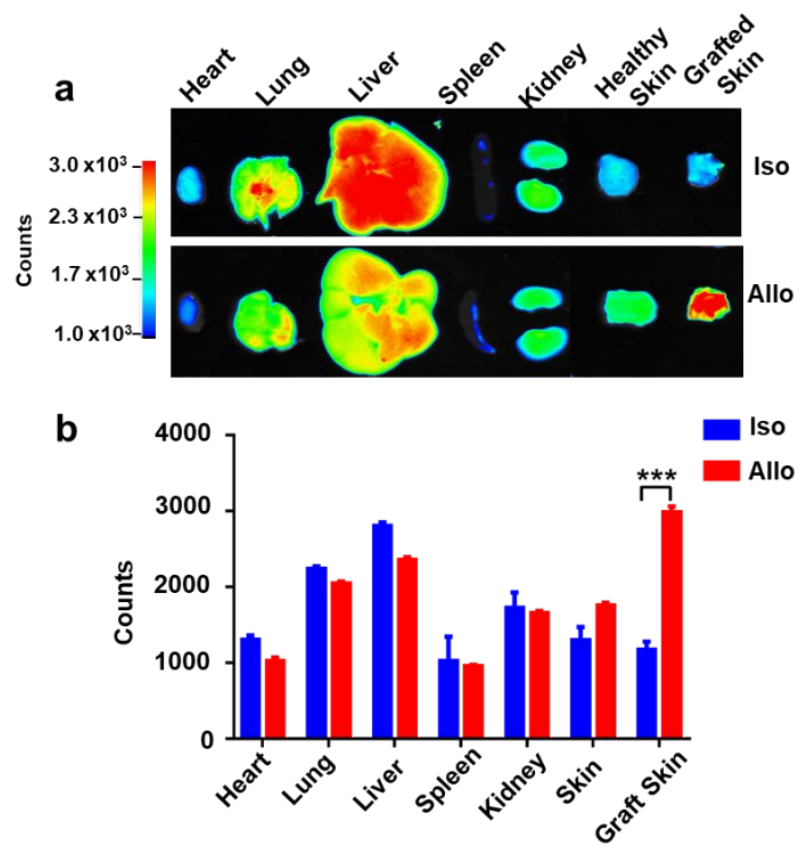

Figure S58. Tissue distribution of allograft mice after oral HBTTPEP/GPs. (a) Representative ex vivo fluorescence image of grafted skin and other organs after oral HBTTPEP/GPs for 24 hours. The organs were imaged with Bruker imaging system (excitation/emission $=510 / 700 \mathrm{~nm}$ ). (b) Biodistribution of HBTTPEP/GPs in 
different organs. All bars represent mean \pm s.d. $(n=3)$. Statistical significance was examined by two-sided Student's t test $(* * * \mathrm{P}<0.001)$.

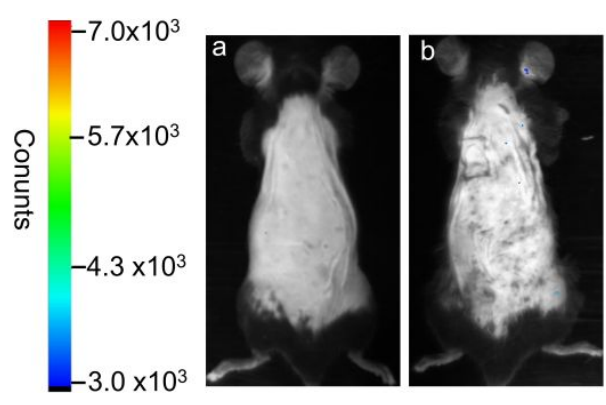

Figure S59. In vivo fluorescence imaging after oral administration of HBTTPEP/GPs in healthy and trauma mice (a) Fluorescent imaging after oral administration of HBTTPEP/GPs for $24 \mathrm{~h}$ in healthy mice; (b) Fluorescent imaging after oral administration of HBTTPEP/GPs for $24 \mathrm{~h}$ in mice of surgical trauma $(\mathrm{n}=3)$.

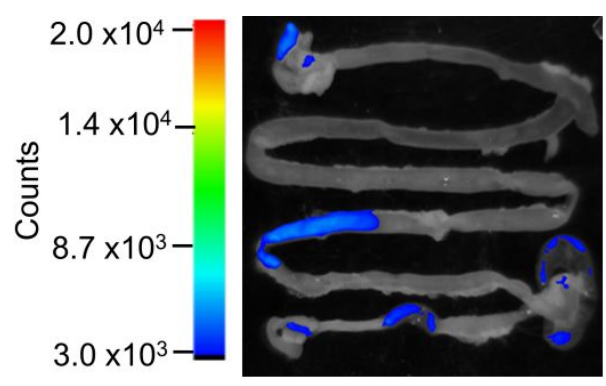

Figure S60. The ex vivo imaging of gastrointestinal tract in healthy mice. $\lambda \operatorname{ex}=510$ $\mathrm{nm} ; \lambda \mathrm{em}=700 \mathrm{~nm}$.
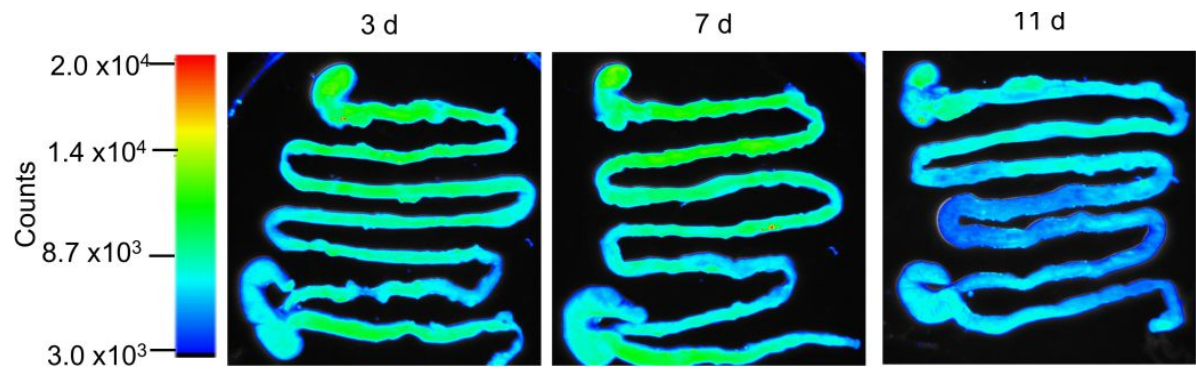

Figure S61. The ex vivo imaging of GI following oral administration of HBTTPEP/GPs at $3 \mathrm{~d}, 7 \mathrm{~d}$ and $11 \mathrm{~d}$. $\lambda \mathrm{ex}=510 \mathrm{~nm} ; \lambda \mathrm{em}=700 \mathrm{~nm}$. 


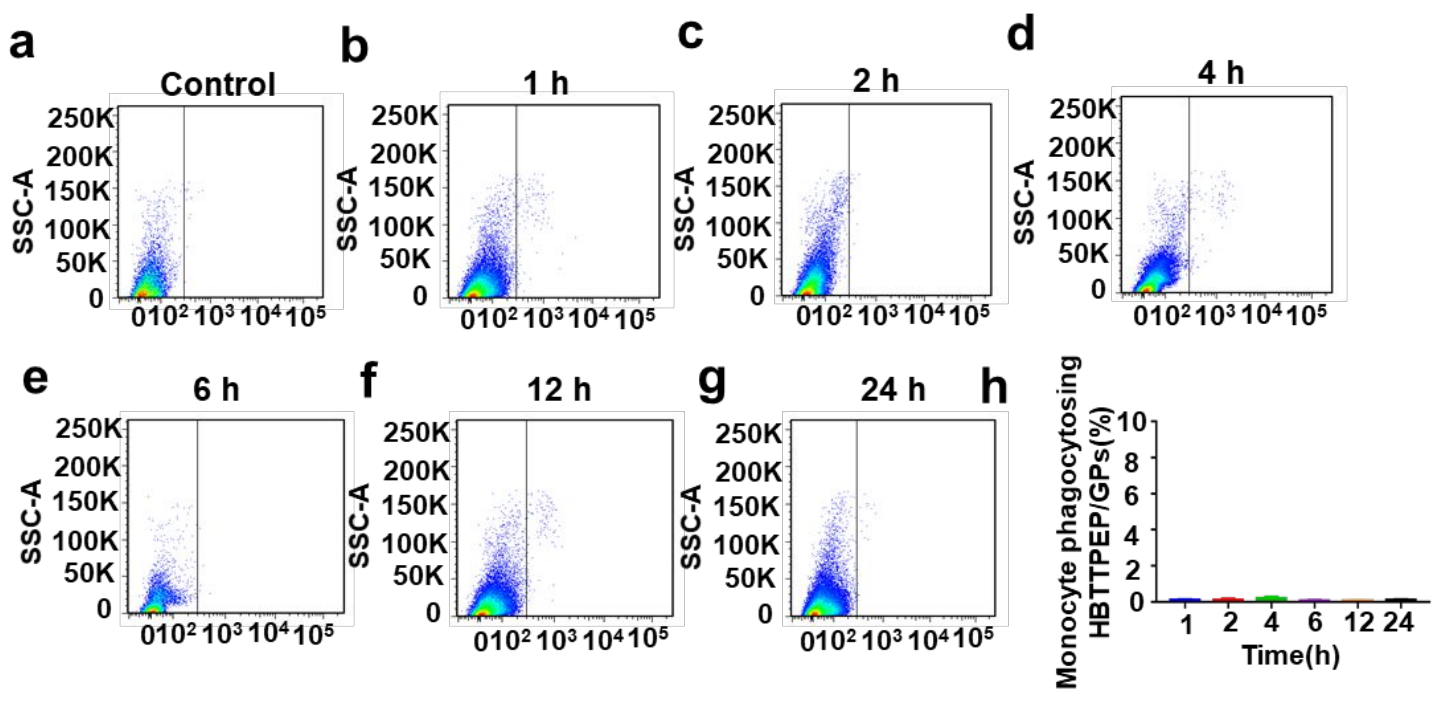

Figure S62. Flow cytometric analysis of peripheral blood monocytes after oral HBTTPEP/GPs in allograft mice at different time points. (a) Flow cytometric analysis of peripheral blood monocytes in allograft mice. (b) Flow cytometric analysis of peripheral blood monocytes $1 \mathrm{~h}$ after oral HBTTPEP/GPs in allograft mice. (c) $2 \mathrm{~h}$ after oral HBTTPEP/GPs. (d) $4 \mathrm{~h}$ after oral HBTTPEP/GPs (e) $6 \mathrm{~h}$ after oral HBTTPEP/GPs. (f) $12 \mathrm{~h}$ after oral HBTTPEP/GPs. (g) $24 \mathrm{~h}$ after oral HBTTPEP/GPs. (h) Quantitative analysis of the ratio of monocytes phagocytosing HBTTPEP/GPs in peripheral blood.

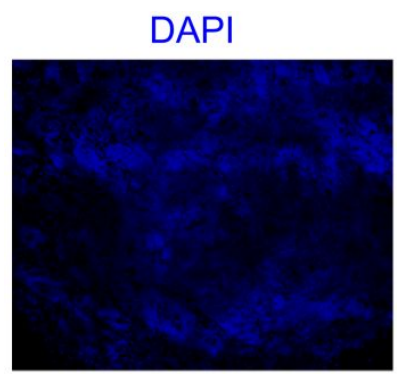

HBTTPEP/GPS
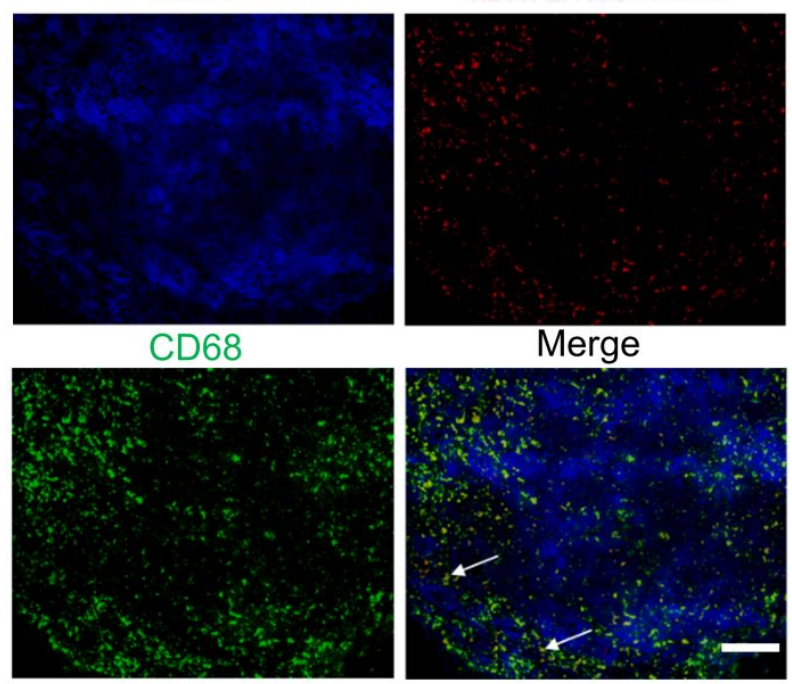

Figure S63. Immunofluorescence images showing co-localization of HBTTPEP/GPs in macrophages of mesenteric lymph nodes isolated from allograft mice after oral administration of HBTTPEP/GPs for $24 \mathrm{~h}$. FITC-labeled antibody was used to stain macrophages, while nuclei were stain with DAPI. Arrow indicates co-localization 
between red fluorescence of HBTTPEP/GPs and green fluorescence of FITC-labeled CD68 antibody; Scale bare $50 \mu \mathrm{m}$.

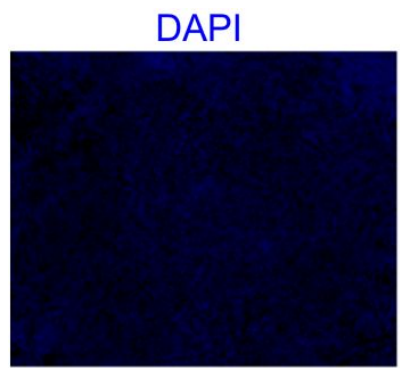

CD68

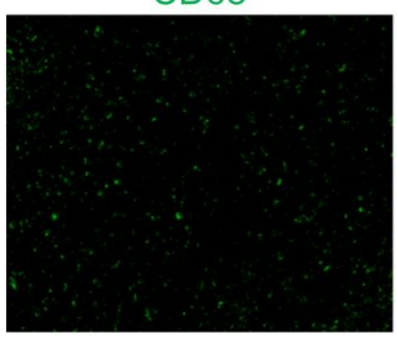

HBTTPEP/GPS

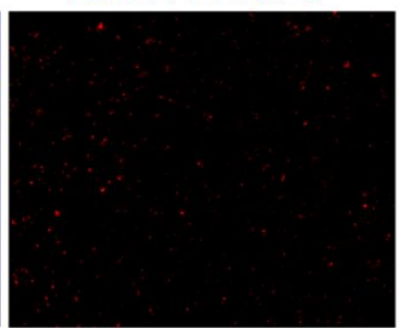

Merge

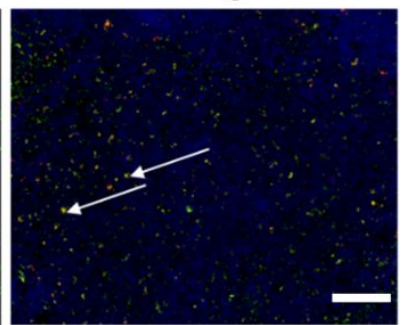

Figure S64. Immunofluorescence images showing co-localization of HBTTPEP/GPs in macrophages of draining lymph nodes isolated from allograft mice after oral administration of HBTTPEP/GPs for $24 \mathrm{~h}$. FITC-labeled antibody was used to stain macrophages, while nuclei were stain with DAPI. Arrow indicates co-localization between red fluorescence of HBTTPEP/GPs and green fluorescence of FITC-labeled anti-CD68 antibody; Scale bare $50 \mu \mathrm{m}$.
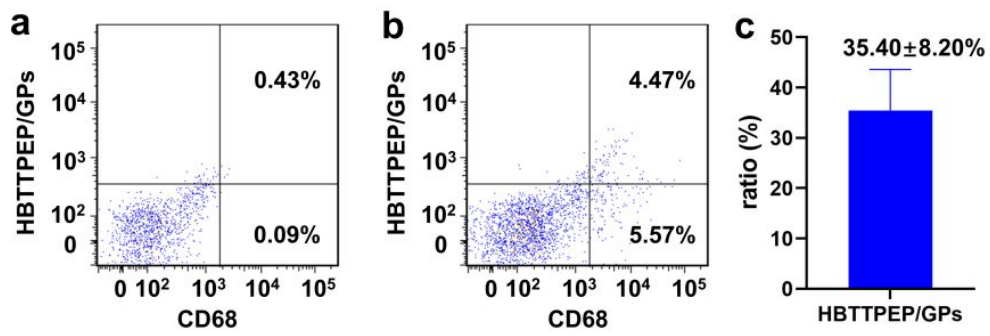

Figure S65. (a) Isolated the cells seven days after allogenic transplantation and perform flow cytometric analysis. (b) Allogeneic transplanted mice were orally administered HBTTPEP/GPs on the 6th day of transplantation. After 24 hours, the cells in the transplant were separated and incubated with PE Anti-CD68 antibody, followed by flow cytometric analysis. (c) The proportion of macrophages phagocytosing HBTTPEP/GPs in the transplanted skin to the total number of CD68 ${ }^{+}$macrophages in graft. 

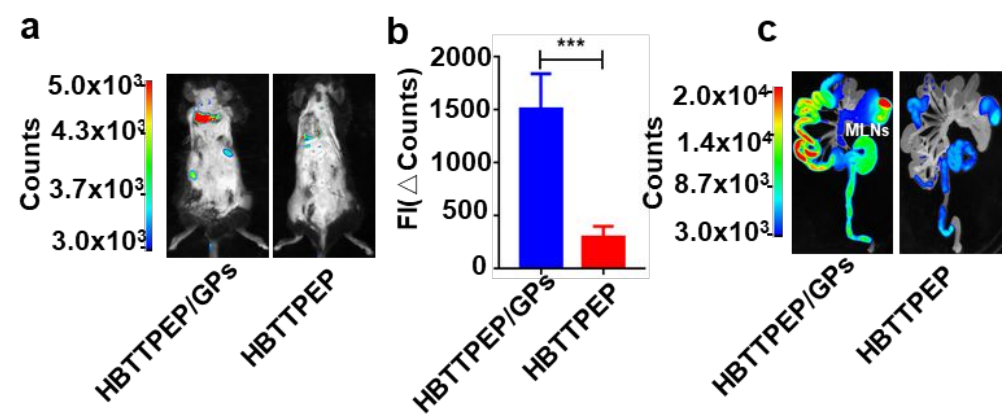

Figure S66. In vivo and ex vivo fluorescence imaging. (a) Fluorescent imaging of allograft mice $(\mathrm{POD}=6)$ after oral administration of HBTTPEP/GPs or HBTTPEP for 24 h. (b) Evolution of the signal intensity in the grafted skin. (c) Ex vivo fluorescence signal of GI tract excised from the allograft mice orally administrated with HBTTPEP/GPs or HBTTPEP.

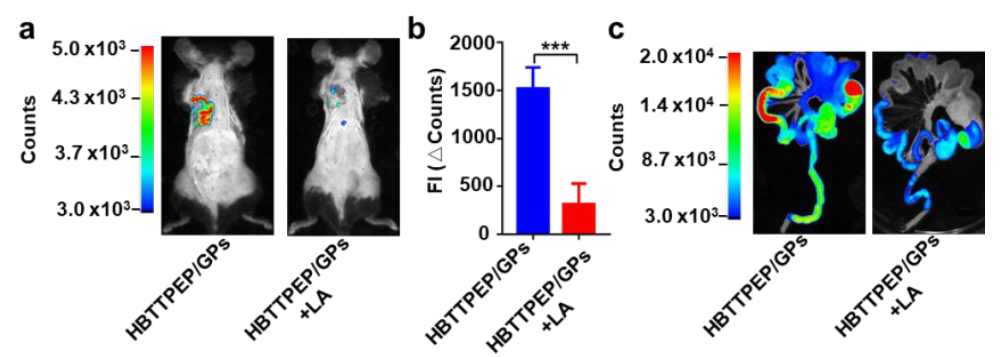

Figure S67. In vivo and ex vivo fluorescence imaging. (a) In vivo fluorescence images of allograft mice after oral administration of the HBTTPEP/GPs or LA $(150 \mathrm{mg} / \mathrm{kg})$ followed by HBTTPEP/GPs. (b) Evolution of the signal intensity in the grafted skin. (c) Distribution of HBTTPEP/GPs in GI tract after oral administration of the HBTTPEP/GPs or LA followed by HBTTPEP/GPs.

\section{Reference}

(1) Gao, T.; Yang. S.; Cao. X.; Dong, J.; Zhao, N.; Ge, P.; Zeng, W.; Cheng, Z. Smart Self-Assembled Organic Nanoprobe for Protein-Specific Detection: Design, Synthesis, Application, and Mechanism Studies. Anal, Chem. 2017, 89, 10085-10093.

(2) Gao, T.; Zeng, H.; Xu, H.; Gao, F.; Li, W.; Zhang, S.; Liu, Y.; Luo, G.; Li, M.; Jiang, D.; Chen, Z.; Wu, Y.; Wang, W.; Zeng, W. Novel Self-Assembled Organic Nanoprobe for Molecular Imaging and Treatment of Gram-Positive Bacterial 
Infection. Theranostics 2018, 8, 1911-1922.

(3) Salomon-Ferrer, R.; Case, D. A.; Walker, R. C. An Overview of the Amber Biomolecular Simulation Package. Wiley Interdiscip. Rev.Comput. Mol. Sci. 2013, 3, $198-210$.

(4) Abraham, M. J.; Murtola, T.; Schulz, R.; Páll, S.; Smith, J. C.; Hess, B.; Lindahl,

E. GROMACS: High Performance Molecular Simulations through Multi-Level Parallelism from Laptops to Supercomputers. SoftwareX 2015, 1-2, 19-25.

(5) Jorgensen, W. L.; Chandrasekhar, J.; Madura, J. D.; Impey, R. W.; Klein, M. L. Comparison of Simple Potential Functions for Simulating Liquid Water. J. Chem. Phys. 1983, 79, 926-935.

(6) Cendales, L. C.; Kanitakis, J.; Schneeberger, S.; Burns, C.; Ruiz, P.; Landin, L.; Remmelink, M.; Hewitt, C. W.; Landgren, T.; Lyons, B.; Drachenberg, C. B.; The Banff 2007 Working Classification of Skin-Containing Composite Tissue Allograft Pathology. Am. J. Transplant 2008, 8, 1396-400. 